\title{
Quantification of Myocardial Blood Flow and Flow Reserve with Rubidium-82 Positron Emission Tomography Imaging
}

\author{
by \\ Jennifer Renaud, B.Eng.

\begin{abstract}
A Thesis Submitted to
in Partial Fulfillment of

the Requirements for the Degree of

Master of Science

Ottawa-Carleton Institute for Physics

Department of Physics, Carleton University

Ottawa, Ontario, Canada

Submitted August, 2008
\end{abstract} \\ the Faculty of Graduate Studies and Research \\ Ccopyright \\ 2008, Jennifer Renaud
}




$\begin{array}{ll}\begin{array}{l}\text { Library and } \\ \text { Archives Canada }\end{array} & \begin{array}{l}\text { Bibliothèque et } \\ \text { Archives Canada }\end{array} \\ \begin{array}{l}\text { Published Heritage } \\ \text { Branch }\end{array} & \begin{array}{l}\text { Direction du } \\ \text { Patrimoine de l'édition }\end{array} \\ \begin{array}{l}\text { 395 Wellington Street } \\ \text { Ottawa ON K1A 0N4 } \\ \text { Canada }\end{array} & \begin{array}{l}\text { 395, rue Wellington } \\ \text { Ottawa ON K1A 0N4 } \\ \text { Canada }\end{array}\end{array}$

Your file Votre référence ISBN: 978-0-494-44138-1 Our file Notre référence ISBN: 978-0-494-44138-1

NOTICE:

The author has granted a nonexclusive license allowing Library and Archives Canada to reproduce, publish, archive, preserve, conserve, communicate to the public by telecommunication or on the Internet, loan, distribute and sell theses worldwide, for commercial or noncommercial purposes, in microform, paper, electronic and/or any other formats.

The author retains copyright ownership and moral rights in this thesis. Neither the thesis nor substantial extracts from it may be printed or otherwise reproduced without the author's permission.
AVIS:

L'auteur a accordé une licence non exclusive permettant à la Bibliothèque et Archives Canada de reproduire, publier, archiver, sauvegarder, conserver, transmettre au public par télécommunication ou par l'Internet, prêter, distribuer et vendre des thèses partout dans le monde, à des fins commerciales ou autres, sur support microforme, papier, électronique et/ou autres formats.

L'auteur conserve la propriété du droit d'auteur et des droits moraux qui protège cette thèse. $\mathrm{Ni}$ la thèse ni des extraits substantiels de celle-ci ne doivent être imprimés ou autrement reproduits sans son autorisation.
In compliance with the Canadian Privacy Act some supporting forms may have been removed from this thesis.

While these forms may be included in the document page count, their removal does not represent any loss of content from the thesis.
Conformément à la loi canadienne sur la protection de la vie privée, quelques formulaires secondaires ont été enlevés de cette thèse.

Bien que ces formulaires aient inclus dans la pagination, il n'y aura aucun contenu manquant.

\section{Canada}




\section{Abstract}

Myocardial blood flow (MBF) and flow reserve (MFR) are important prognostic markers for patients with heart disease. By comparing patient scans with a database defining the lower limit of normal MBF or MFR, diagnosis may be improved. The clinical standard for flow measurement is nitrogen-13-ammonia $\left({ }^{13} \mathrm{NH}_{3}\right)$ positron emission tomography imaging, necessitating an expensive cyclotron. A cost-effective alternative is the generator-produced tracer rubidium- $82\left({ }^{82} \mathrm{Rb}\right) .{ }^{82} \mathrm{Rb}$ is commonly used for relative MBF imaging; its accuracy for absolute MBF imaging has not been widely assessed. Tracer kinetic methods for quantifying MBF with ${ }^{82} \mathrm{Rb}$, including compartment and net retention models, were developed and validated versus ${ }^{13} \mathrm{NH}_{3}$ measurements. Normal population databases were created and used for patient evaluation. The results support MBF quantification with ${ }^{82} \mathrm{Rb}$, and suggest that compartment modeling with ${ }^{13} \mathrm{NH}_{3}$ remains most precise for quantifying absolute flow, whereas the retention model is most accurate for detection and localization of abnormal MBF. 


\section{Statement of Originality}

Portions of the work discussed in this thesis were presented in poster form at the IEEE Nuclear Science Symposium and Medical Imaging Conference (IEEE NSS/MIC) in 2007, as well as in oral presentation format at the Society of Nuclear Medicine (SNM) Annual Meeting in 2008. This work was also published as a short manuscript in the conference proceedings of the 2007 IEEE NSS/MIC [1] and as an abstract for the 2008 SNM Annual Meeting [2]. My contribution to the work published in [1] consisted of creating the analysis methods and performing the analysis, as well as composing the first draft of the manuscript. The coauthors participated in reviewing the short manuscript. For the second contribution [2] I implemented the methods and performed the analysis of the data, as presented in my thesis. Additionally, I created the figures and slides for the presentation. The coauthors contributed by reviewing the abstract and providing feedback for the presentation. 


\section{Acknowledgements}

I would like to thank my supervisor, Dr. Rob deKemp, for his mentorship throughout my studies and research at the University of Ottawa Heart Institute. His guidance and support allowed me to successfully complete this research project, while learning many new concepts and skills that will be assets in my future pursuits and challenges.

My heartfelt appreciation goes to my family and friends for their constant support and understanding throughout the duration of my studies and research, as well as their patience during the writing of this thesis. Without them, this body of work would not have been possible.

I am also grateful to my fellow students and the employees in the cardiac PET group, whose assistance, encouragement and camaraderie made my experience both productive and rewarding. 


\section{Table of Contents}

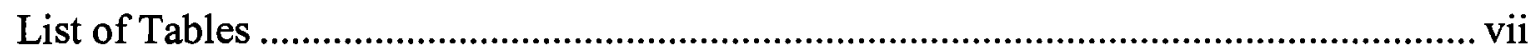

List of Figures .......................................................................................................... viii

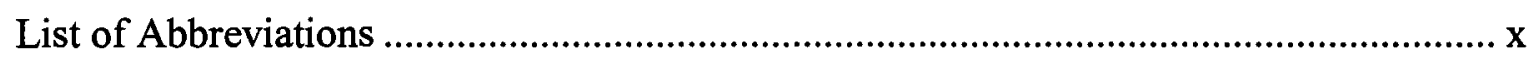

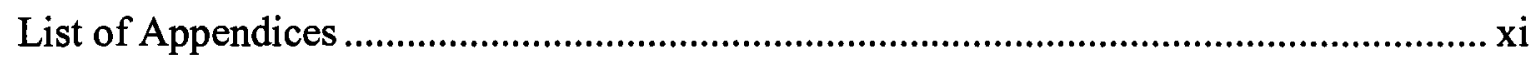

CHAPTER 1 - Positron Emission Tomography for Cardiac Imaging.......................... 1

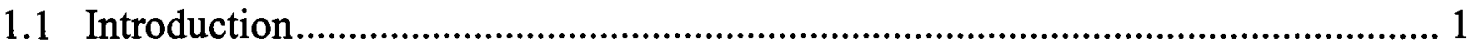

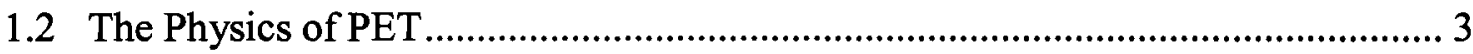

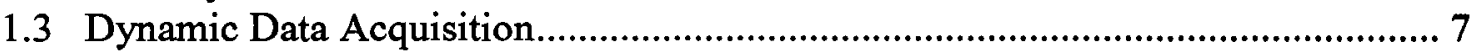

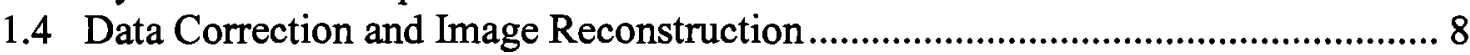

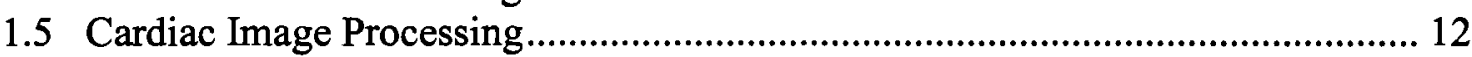

1.6 Myocardial Blood Flow and Flow Reserve ................................................... 19

1.7 PET Tracers for Blood Flow Imaging .............................................................. 19

1.8 Relative Myocardial Perfusion Imaging with PET ................................................ 22

1.9 Absolute Myocardial Blood Flow Imaging with PET ..................................... 22

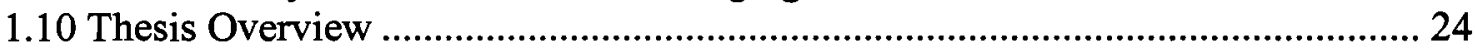

CHAPTER 2 - Tracer Kinetic Modeling.................................................................................. 25

2.1 Principles of Kinetic Modeling ..................................................................... 25

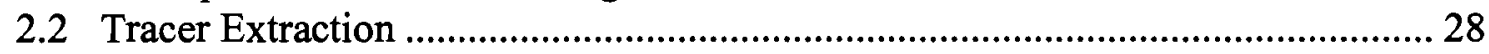

2.3 Two-Compartment Models.................................................................................... 30

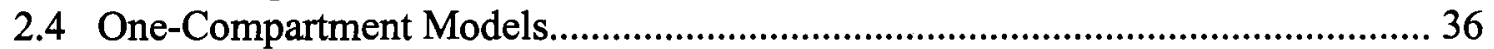

2.5 Implementation of Compartment Models ................................................................ 38

CHAPTER 3 - Development and Validation of a Simplified Net Retention Model. 40

3.1 Principles of the Simplified Net Retention Model.................................................... 40

3.2 Determination of a Correction for Partial Volume Losses ................................ 42

3.3 Optimization of the Arterial Blood Integration Time .......................................... 42

3.4 Development of Net Extraction Corrections.............................................................. 46

3.5 Validation of Net Extraction Corrections ..................................................... 51

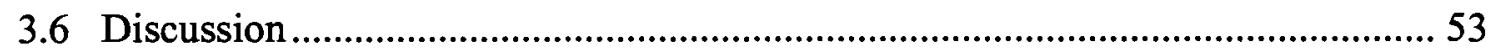

CHAPTER 4 - Criteria and Construction of Normal Population Databases........... 57

4.1 Purpose and Utility of Normal Population Databases ............................................ 57

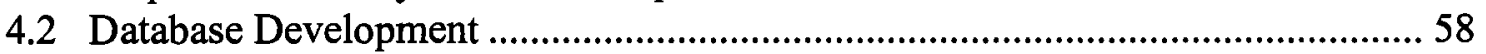

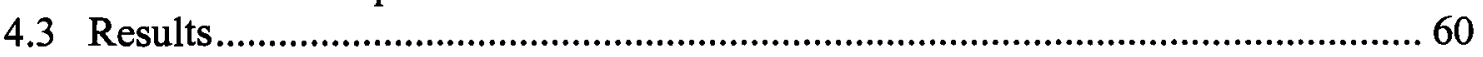

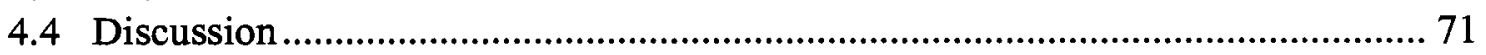

CHAPTER 5 - Evaluation of Patients Using Normal Population Databases ............ 74

5.1 Characterization of the Patient Population ........................................................... 74

5.2 Receiver Operating Characteristic Analysis ......................................................... 75

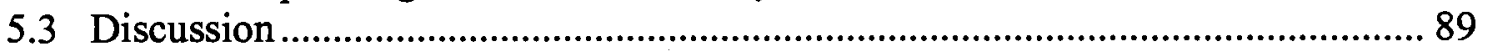

CHAPTER 6 - Conclusion ............................................................................................ 96 


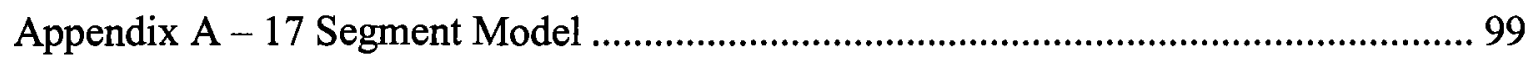

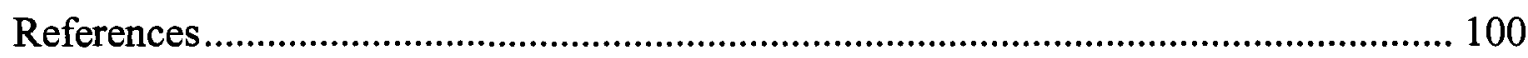




\section{List of Tables}

Table 1.1: Positron energies and ranges for common PET isotopes................................ 4

Table 1.2: Commonly used PET tracers ..................................................................... 20

Table 3.1: Mean retention values derived from retention for the gamma variate and the

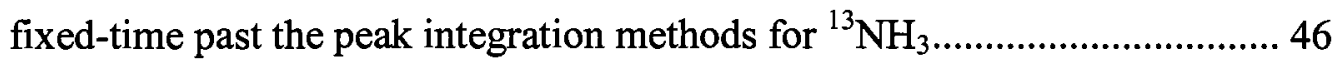

Table 4.1: Global (LV-median) normal ranges and lower limits of normal for the retention and compartment models...

Table 4.2: Regional (polar map) normal ranges and lower limits of normal for the retention and compartment models..........................................................6 62

Table 4.3: Regional uniformity of the database polar maps .......................................69

Table 4.4: Sample standard deviation of random normal distributions .......................... 70

Table 5.1: SD thresholds and minimum percent defect size cutoffs with corresponding sensitivity and specificity for each region using the relative retention database

Table 5.2: SD thresholds and minimum percent defect size cutoffs with corresponding sensitivity and specificity for each region using the absolute retention database.

Table 5.3: Summary of literature regarding the accuracy of ${ }^{82} \mathrm{Rb}$ databases for detection of CAD. 


\section{List of Figures}

Figure 1.1: Illustration of positron decay, an LOR and the types of coincidences detected in PET imaging

Figure 1.2: (A) Transaxial, (B) VLA, and (C) HLA slices of the myocardium .............. 13

Figure 1.3: Sampling of the SA slices ................................................................. 14

Figure 1.4: Sampling of the (A) VLA and (B) HLA …….......................................... 14

Figure 1.5: (A) 2D representation of the LV in polar map format. (B) 3D view of the same data that is presented in the polar map.................................................... 15

Figure 1.6: Blood and myocardial TACs................................................................ 15

Figure 1.7: $\mathrm{PV}$ averaging in a simulated $10 \mathrm{~mm}$ thick uniform myocardial wall with 10 mm scanner resolution ............................................................................. 18

Figure 1.8: ${ }^{82} \mathrm{Rb}$ VLA image of the LV from a normal subject........................................ 18

Figure 2.1: A generalized two-compartment model ...................................................... 26

Figure 2.2: Renkin-Crone capillary model of tracer extraction...................................... 29

Figure 2.3: Uptake of flow tracers in the myocardium as a function of MBF................ 30

Figure 2.4: Two-compartment model describing the uptake and retention of ${ }^{13} \mathrm{NH}_{3}$ in

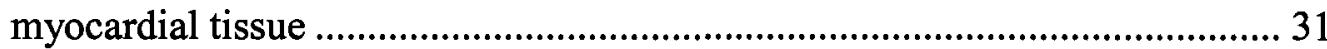

Figure 2.5: Alternate two-compartment model for ${ }^{13} \mathrm{NH}_{3}$........................................... 34

Figure 2.6: One-compartment model describing the uptake and retention of ${ }^{13} \mathrm{NH}_{3}$ in

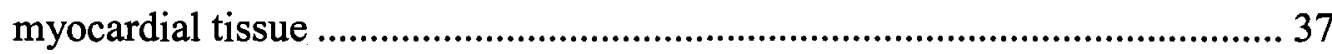

Figure 3.1: Simplified net retention model describing the uptake and retention of activity

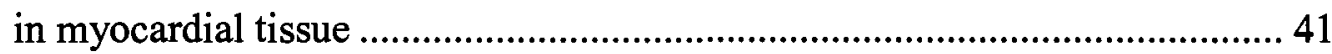

Figure 3.2: Gamma variate fit to the arterial blood input curve of a normal subject........ 44

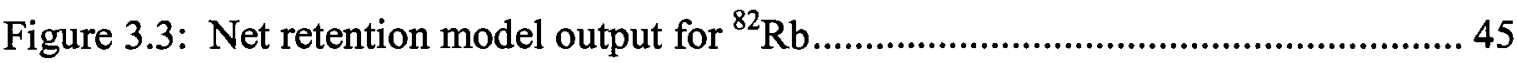

Figure 3.4: (A) Derived extraction curves. (B) Net retention $(E \times M B F)$ curves ............. 49

Figure 3.5: Comparison of ${ }^{13} \mathrm{NH}_{3}$ and ${ }^{82} \mathrm{Rb}$ net retention at stress for an individual

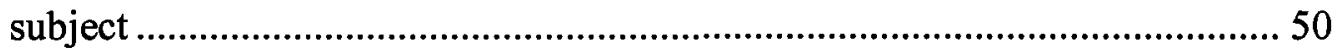

Figure 3.6: Extraction curves for CAD patients compared with the curves from healthy volunteers

Figure 3.7: Comparison of extraction curves from previous studies in animals and the

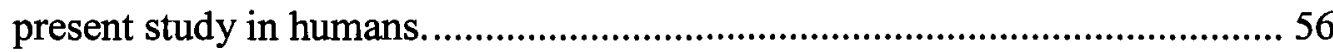

Figure 4.1: Regional polar maps of the normal population mean..................................... 64 
Figure 4.2: Comparison of the mean values from the retention and compartmental model databases for (A) ${ }^{82} \mathrm{Rb}$ and (B) ${ }^{13} \mathrm{NH}_{3}$ 66

Figure 4.3: Comparison of the variability from the retention and compartmental model databases for (A) ${ }^{82} \mathrm{Rb}$ and (B) ${ }^{13} \mathrm{NH}_{3}$ 67

Figure 4.4: Rest, stress and stress/rest polar maps in a subject with CAD 73

Figure 5.1: ROC curves for a perfectly accurate diagnostic test, a moderately accurate test, and the chance diagonal

Figure 5.2: Division of the polar map into three vascular territories: LAD, LCX, and RCA.

Figure 5.3: Whole $\mathrm{LV}$ and regional $\mathrm{AUC}$ versus $\mathrm{SD}$ threshold values for the relative ${ }^{82} \mathrm{Rb}$ databases

Figure 5.4: Whole LV and regional AUC versus SD threshold values for the absolute ${ }^{82} \mathrm{Rb}$ databases. 82

Figure 5.5: Characteristic pattern of regional ROC curves at varying SD thresholds..... 84 Figure 5.6: Whole LV curve at an SD threshold of 2.5, and regional ROC curves at an $\mathrm{SD}$ threshold of 2 , for the relative ${ }^{82} \mathrm{Rb}$ databases. 87

Figure 5.7: Whole LV and LAD ROC curves at an SD threshold of 2, and LCX and RCA ROC curves at an SD threshold of 2.5 for the absolute ${ }^{82} \mathrm{Rb}$ databases. 88

Figure 5.8: Polar maps showing the evaluation of a CAD patient with the retention database... 94

Figure A.1: Polar map of the 17 segment model. 99 


\section{List of Abbreviations}

$\begin{array}{ll}\text { AUC } & \text { area under the curve } \\ \text { CAD } & \text { coronary artery disease } \\ \text { COV } & \text { coefficient of variation } \\ \text { CT } & \text { computed tomography } \\ \text { E } & \text { extraction correction } \\ \text { FBP } & \text { filtered back projection } \\ \text { FDG } & \text { fluorodeoxyglucose } \\ \text { FN } & \text { false negative } \\ \text { FOV } & \text { field of view } \\ \text { FP } & \text { false positive } \\ \text { FPR } & \text { false positive rate } \\ \text { HLA } & \text { horizontal long axis } \\ \text { LAD } & \text { left anterior descending coronary artery } \\ \text { LCX } & \text { left circumflex coronary artery } \\ \text { LOR } & \text { line of response } \\ \text { LV } & \text { left ventricle } \\ \text { MBF } & \text { myocardial blood flow } \\ \text { MFR } & \text { myocardial flow reserve } \\ \text { MPI } & \text { myocardial perfusion imaging } \\ \text { 13NH } & \text { nitrogen-13-ammonia } \\ \text { PET } & \text { positron emission tomography } \\ \text { PS } & \text { capillary permeability-surface area product } \\ \text { PSF } & \text { point spread function } \\ \text { PV } & \text { partial volume } \\ \text { R } & \text { net retention rate } \\ \text { RC } & \text { recovery coefficient } \\ \text { RCA } & \text { right coronary artery } \\ \text { RMS } & \text { root mean square } \\ \text { ROC } & \text { receiver operating characteristic } \\ \text { ROI } & \text { region of interest } \\ \text { SA } & \text { short axis } \\ \text { SPC } & \text { specificity } \\ \text { SD } & \text { standard deviation } \\ \text { SEN } & \text { sensitivity } \\ \text { SPC } & \text { specificity } \\ \text { SPECT } & \text { single-photon emission computed tomography } \\ \text { TAC } & \text { time-activity curve } \\ \text { TN } & \text { true negative } \\ \text { TP } & \text { true positive } \\ \text { TPR } & \text { true positive rate } \\ \text { UOHI } & \text { University of Ottawa Heart Institute } \\ \text { VLA } & \text { vertical long axis } \\ & \end{array}$




\section{List of Appendices}

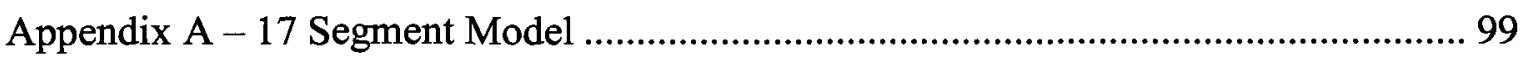




\section{CHAPTER 1 - Positron Emission Tomography for Cardiac Imaging}

\subsection{Introduction}

Positron emission tomography (PET) is a highly sensitive, relatively non-invasive 3D imaging modality used to assess physiological function by measuring the activity of biological compounds in a specific region of the body, such as the heart. To trace a compound it is labelled with a positron-emitting isotope, creating a tracer. The tracer is administered to the subject and subsequently taken up into the tissue of interest where the isotope decays over a short period of time. Decay events are detected by the PET scanner and are reconstructed to form tomographic images of the tracer distribution inside the body; these images are representative of biological processes occurring within the tissues of interest.

Due to its functional imaging capabilities, PET has found applications as both a clinical diagnostic and research tool, primarily in the areas of cardiology, oncology, and neurology. It is also used in pharmacology for the investigation of new drugs. In cardiology, PET is used to assess characteristics of the heart, particularly of the left ventricle (LV), which is the primary tissue of interest as it is the main pumping chamber. Such characteristics include the blood flow to the LV tissue (myocardium) as a means of identifying and diagnosing a number of heart diseases. Specifically, PET permits the quantitative measurement of absolute myocardial blood flow (MBF) in units of 
$\mathrm{mL} / \mathrm{min} / \mathrm{g}$. This measurement provides useful information for the management of patients with global impairment of LV blood flow and myocardial flow reserve, conditions which have been associated with multi-vessel coronary artery disease (CAD), microvascular disease, hypertension, hyperlipidemia, and heart failure following transplant.

At present, the clinical standard for the measurement of absolute MBF is nitrogen-13ammonia $\left({ }^{13} \mathrm{NH}_{3}\right)$ PET imaging. Due to the short half-life of ${ }^{13} \mathrm{NH}_{3}$, imaging of this type requires on-site tracer production with a cyclotron, a procedure that is both complex and costly. As such, a demand for quantification methodology without the need for a cyclotron exists. At the University of Ottawa Heart Institute (UOHI), methods are being developed for PET imaging using the easily transportable, generator-produced tracer rubidium-82 $\left({ }^{82} \mathrm{Rb}\right)$. These methods include strontium-82 $\left({ }^{82} \mathrm{Sr}\right) /{ }^{82} \mathrm{Rb}$ generator manufacturing, ${ }^{82} \mathrm{Rb}$ imaging and analysis $[3,4,5,6]$. Historically, this tracer has been used for relative myocardial perfusion imaging (MPI). Relative imaging methods are effective in detecting significant coronary disease in cases where at least one or more regions of the heart have normal perfusion, allowing for comparison with suspected diseased regions. However, in cases where a normal region is absent, or where mild disease may be present prior to symptom development, quantification of absolute MBF may help to correctly identify and localize any regional flow abnormalities. Validation of the accuracy of absolute MBF measurements with ${ }^{82} \mathrm{Rb}$ has not been widely reported. Therefore, the objectives of this research were to develop tracer kinetic methods for the quantification of $\mathrm{MBF}$ with ${ }^{82} \mathrm{Rb} \mathrm{PET}$, and to validate these methods versus measurements made with ${ }^{13} \mathrm{NH}_{3}$ on the ECAT ART 3D PET system (CTI/Siemens, 
Knoxville, TN). Following validation of the modeling methods, the goal was to form normal population databases defining the lower limit of normal MBF in an attempt to further aid in the automated diagnosis and localization of CAD. The results of this work are anticipated to support more widespread use of absolute MBF measurements with ${ }^{82} \mathrm{Rb}$ PET, particularly in centres without a cyclotron.

\subsection{The Physics of PET}

PET is the leading tool in nuclear cardiology for the non-invasive assessment of cardiac molecular function. Using this modality, images of physiological processes can be obtained by injecting a subject with either a natural biological compound, or an analog of a naturally-occurring substance, labeled with a positron-emitting isotope. This labeled compound is referred to as a tracer. The radioactive isotopes used in PET tracers have an excess of protons, causing them to decay by positron emission $\left(\beta^{+}\right)$into stable forms according to the following scheme:

${ }_{Z}^{A} X \rightarrow{ }_{Z-1}^{A} Y+\beta^{+}+v_{e}$

where $X / Y$ are the parent/daughter isotopes with atomic mass $A$ and atomic number $Z$, and $\nu_{e}$ represents an electron neutrino that is also emitted in this process. For this decay to occur, the difference in rest mass energies between the parent and daughter nuclei must exceed $1.022 \mathrm{MeV}$, or $2 m_{e} c^{2}$, where $m_{e}$ is the mass of an electron. The energy remaining from this process is shared as kinetic energy between the neutrino and the $\beta^{+}$; root mean square (RMS) and maximum positron energies for common PET isotopes are shown in Table 1.1. Subsequent to its emission, the positron will travel through the surrounding 
tissue, losing energy in inelastic collisions with neighbouring electrons until its energy is reduced sufficiently; at this point it will annihilate with an electron. Positron ranges in water are given in Table 1.1. The $\beta^{+} / e^{-}$annihilation results in the production of two colinear $\left(180^{\circ}\right) 511 \mathrm{keV}$ photons, or gamma rays $(\gamma)$, which propagate in opposite directions according to the principles of energy and momentum conservation (Fig. 1.1). Note that if the residual momentum of the annihilation particles is non-negligible, the resultant annihilation photons will not be emitted in exact co-linearity, but will deviate by a standard deviation of $\sim 0.5^{\circ}[7]$.

Table 1.1: Positron energies and ranges for common PET isotopes [8]

\begin{tabular}{c|c|c|c|c}
\hline Isotope & $\begin{array}{c}\text { Max Energy } \\
(\mathrm{MeV})\end{array}$ & $\begin{array}{c}\text { Mean Energy } \\
(\mathrm{MeV})\end{array}$ & $\begin{array}{c}\text { Max Range } \\
(\mathrm{mm})\end{array}$ & $\begin{array}{c}\text { RMS Range } \\
(\mathrm{mm})\end{array}$ \\
\hline${ }^{18} \mathrm{~F}$ & 0.64 & 0.250 & 2.3 & 0.35 \\
${ }^{11} \mathrm{C}$ & 0.96 & 0.386 & 4.1 & 0.56 \\
${ }^{13} \mathrm{~N}$ & 1.19 & 0.492 & 5.2 & 0.72 \\
${ }^{15} \mathrm{O}$ & 1.72 & 0.735 & 8.1 & 1.1 \\
${ }^{82} \mathrm{Rb}$ & 3.35 & 1.52 & 16.7 & 2.4 \\
\hline
\end{tabular}

Once the pair of annihilation photons leaves the body, coincidence detection can be performed. To detect the photons, a PET scanner consisting of a ring of scintillation crystal detectors encircles the subject. The crystals are coupled to photomultiplier tubes that convert incoming scintillation photons to photoelectrons, which can then be detected by the system. When the annihilation photons strike separate detectors, the times of the events are recorded, and subsequently compared, to determine if both events fell within the coincidence timing window. The window is typically on the order of $3-12$ nanoseconds, depending on the detector characteristics as well as the imaging 
application. Any two events recorded at opposing detectors within this window of time are considered to be coincident. This method allows for three types of coincidences to be recorded: true, scattered and random. True coincidences are the result of recording photons that originated from a single decay event and did not undergo any scatter in the medium, whereas a scattered event is recorded when one or both of the photons from a single decay event are scattered by the medium and subsequently misaligned. Compton scattering is the predominant cause of such scattered events in soft tissue due to a high probability of interaction per unit distance of $\mu_{C}=0.096 \mathrm{~cm}^{-1}$ [9]. Coherent scattering is negligible at the $511 \mathrm{keV}$ energy of the annihilation photons, and photoelectric absorption has a very small probability of occurring in this medium since $\mu_{p e}$ is equivalent to $0.00002 \mathrm{~cm}^{-1}$ in soft tissue [9]. According to Eq. 1.2, Compton scattering reduces the overall energy of the photon $(h \nu)$ by transferring a portion of its energy $(h \nu)$ to a recoil electron, and its initial trajectory is altered by $\theta$, resulting in misalignment of the event.

$$
h v^{\prime}=\frac{h v}{1+\frac{h v}{m_{e} c^{2}}(1-\cos \theta)}
$$

The third type of coincidence, random coincidence, is the result of two photons from separate decay events simultaneously striking opposing detectors and being registered as a coincidence event (Fig. 1.1) [10].

A coincident event indicates that an annihilation occurred somewhere along the line between the two detectors, implying that the radioactive atom that decayed must also be located along that same line of response (LOR) (Fig. 1.1). Using this method, coincident 
events are recorded in LORs, defined by a particular radial and angular coordinate corresponding to each possible detector pair. Data from the LORs can then be organized into a histogram, where each bin corresponds to a unique radial and angular location in the field of view (FOV). This histogram is referred to as a sinogram since a point source of activity maps out a half-sine wave [10]. Events can alternatively be stored in list-mode format, where each LOR coordinate, along with the corresponding time and energy of the event, are stored as separate entries. A sinogram is then created post-acquisition. To obtain an image from the sinogram, image reconstruction must be performed. Prior to image reconstruction the data should be corrected to remove the effects of scattered and random coincidences. This will be discussed further in a later section. 


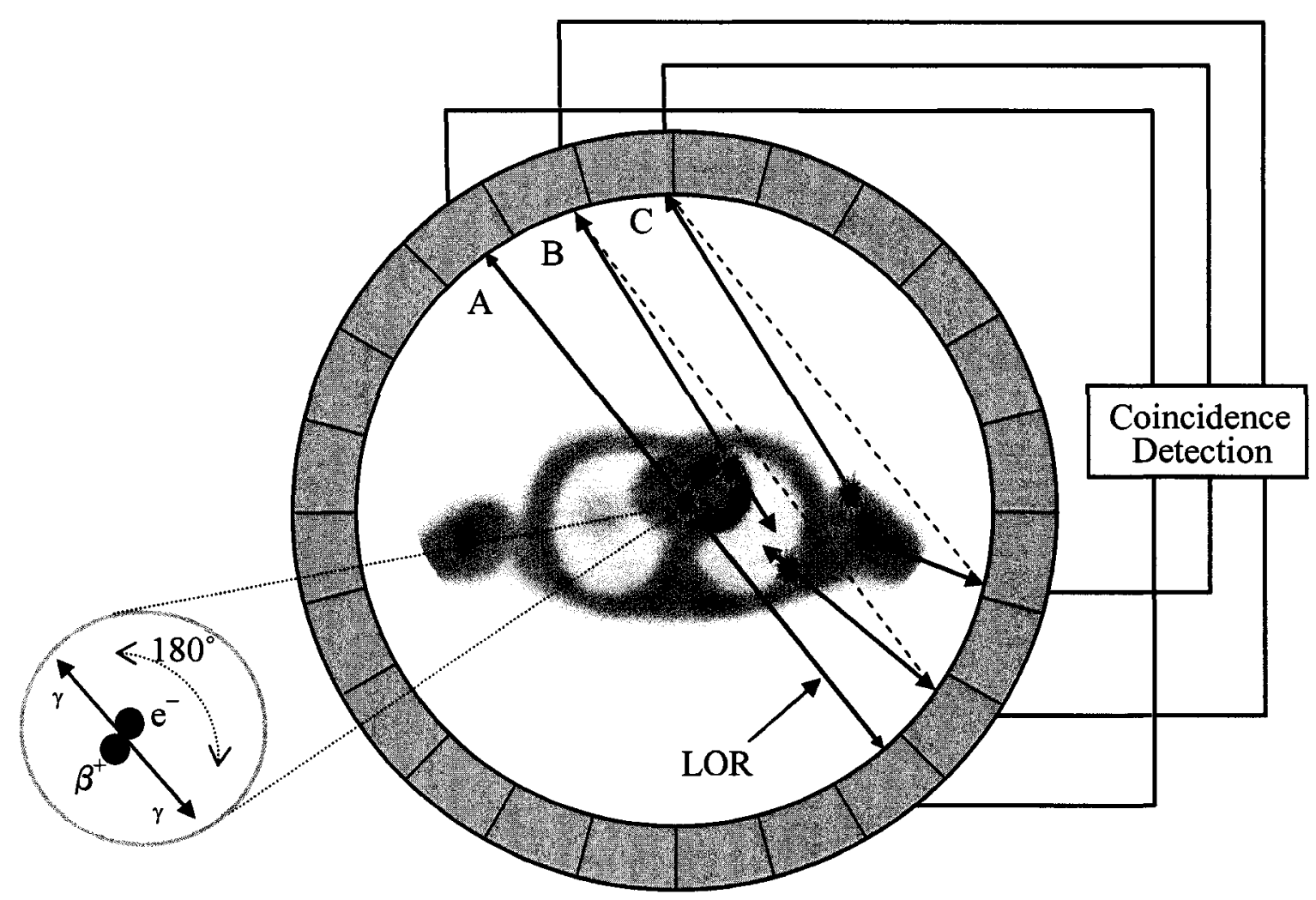

Figure 1.1: Illustration of positron decay, an LOR and the types of coincidences detected in PET imaging. Upon radioisotope decay, a positron is emitted, which travels through the tissue until it annihilates with an electron. The resultant collinear annihilation photons escape from the body and are detected as a coincident event, indicating that the decay occurred along the (LOR). Three types of coincidences can be detected: (A) true, (B) random, and (C) scattered coincidences.

\subsection{Dynamic Data Acquisition}

Coincident datasets can be recorded during two types of acquisitions: static and dynamic.

Static acquisitions involve taking a snapshot of the tracer distribution at one instant in time following injection. Dynamic acquisitions measure the tracer distribution beginning at the time of injection and continuing over a finite time period. PET imaging of 
molecular cardiac function requires dynamic data acquisition to enable the assessment of tracer kinetics for the quantification of parameters of interest, such as blood flow. Typically, these dynamic scans are performed at rest and following pharmacological stress using a vasoldilatory drug to simulate cardiac response under exercise conditions. For instance, at the UOHI, rest and stress dynamic data are acquired on the ECAT ART 3D PET system (CTI/Siemens, Knoxville, TN) with the following acquisition protocols for two blood flow tracers, consisting of $N$ time frames x $M$ frame lengths (seconds): ${ }^{13} \mathrm{NH}_{3}: 12 \times 10 \mathrm{~s}, 2 \times 30 \mathrm{~s}, 1 \times 60 \mathrm{~s}, 1 \times 120 \mathrm{~s}, 1 \times 240 \mathrm{~s}, 2 \times 300 \mathrm{~s}$, total $=20 \mathrm{~min}, 19$ time frames; ${ }^{82} \mathrm{Rb}: 12 \times 10 \mathrm{~s}, 2 \times 30 \mathrm{~s}, 1 \times 60 \mathrm{~s}, 1 \times 120 \mathrm{~s}, 1 \times 240 \mathrm{~s}$, total $=10 \mathrm{~min}, 17$ time frames. Data acquired in this manner are referred to as emission data, since they are obtained by imaging a subject that has been injected with a positron-emitting isotope. PET can also be used to acquire transmission data, which are obtained using an external activity source, similarly to the way an x-ray tube is used on a computed tomography (CT) scanner. Transmission data are used to correct for attenuation of the emission data, as discussed in the subsequent section.

\subsection{Data Correction and Image Reconstruction}

The acquired dynamic data must first be corrected, either during or following the acquisition, for a number of effects in order to obtain a reconstructed image that is truly proportional to the concentration of activity at each location in the object. Detector efficiency, dead time, random and scatter coincidence, attenuation and isotope decay corrections are all generally performed prior to reconstruction. 


\section{Normalization}

Normalization accounts for non-uniformities that arise in the data due to variations in individual detector sensitivity and the variable spaces between detectors resulting from the circular geometry of the scanner. To perform this correction, the number of counts detected by each coincidence pair of detectors is recorded while all of the detector pairs are exposed to the same uniform radiation source placed in the FOV. The inverse of the recorded counts is then multiplied by the previously acquired total scan counts in each LOR to obtain a normalized dataset [10], thus correcting for detector inefficiency.

\section{Dead-Time Correction}

Dead-time refers to the time during which a detector is processing an event and is thus unable to detect any additional events. This limitation causes the count rate to deviate from its expected linear relationship with increasing activity, resulting in an underestimation of tracer activity. There are two common models used to correct for dead-time losses: paralyzable and non-paralyzable. With the paralyzable method, the dead-time is extended if another photon strikes the detector when it is already processing an event, whereas the non-paralyzable method completely ignores the strike that occurs during the dead-time [10].

\section{Random Coincidence Correction}

Random coincidences must be removed as they add a uniform background that distorts the true activity distribution in the image. There are two methods to correct for these unwanted coincidence events. The first involves calculating randoms from the single 
event rates for detector pairs on each LOR. The randoms can then be determined using the following relationship:

$$
R=\tau S_{1} S_{2}
$$

where $\tau$ is the width of the coincidence time window, and $S_{1}$ and $S_{2}$ are the single event rates of the two detectors along a particular LOR. The randoms are then subtracted from the total coincident events recorded along that LOR. The second approach is referred to as the delayed-window method. In this scheme, a second coincidence window that is delayed in time from the initial window, and is much wider, is created. Any coincidences subsequently recorded in this delayed window are then considered to be randoms since the time delay indicates that they cannot have originated from the same decay event. As with the previous method, the randoms are then subtracted from the total coincident rate for all possible LORs [11].

\section{Scatter Correction}

Scattered coincidences are problematic as they increase the background counts in the data, resulting in increased image noise and decreased contrast. A number of these events can be removed using energy discrimination during data acquisition. With an appropriately chosen lower energy threshold, the photons that have reduced energy due to Compton scattering can be identified and discarded; however, this method does not remove all of the scatter events due to the limited energy resolution of the detectors. The most common method of scatter correction involves a direct calculation of the scatter distribution, acquired from the intensity of the emitted photons in an emission image, and knowledge of the attenuation map, from a transmission image. Using the Klein-Nishina 
formula to model the Compton scatter interactions from which the scattered coincidences arise, the correction algorithm iteratively calculates the single scatter events, forming a scatter distribution that can then be subtracted from the total measured coincidences [11].

\section{Attenuation Correction}

As annihilation photons travel through the tissue they can be scattered or absorbed, decreasing the likelihood that they will actually exit the body and strike a detector. The probability that both photons will reach their respective detectors is given by:

$$
P=e^{-\mu x_{1}} e^{-\mu x_{2}}=e^{-\mu\left(x_{1}+x_{2}\right)}
$$

where $\mu$ is the linear attenuation coefficient of tissue for photons of $511 \mathrm{keV}$ energy, and $x_{1}$ and $x_{2}$ are the distances the photons must travel through a uniform tissue before reaching the detector. This effect must be corrected for as it can result in the underestimation of the tracer activity. Since the total attenuation is not dependent on the source location, attenuation factors can be measured accurately using external positron sources. This is achieved by acquiring two scans: a blank scan, without anything present in the FOV, and a transmission scan with the subject in the FOV. In both cases, data is collected while external sources are rotated around the FOV to sample all LORs. Dividing the transmission data by the blank data results in a set of probabilities for detecting coincident events along each LOR; these probabilities are referred to as attenuation factors. To correct for attenuation, the data from the scan of the subject is then simply divided by the attenuation factors [10]. Thus, this general correction does not require knowledge of the specific $\mu$ and $x$ values, and is applicable to any combination of 
the two quantities. To perform attenuation correction on the ECAT ART scanner at the UOHI, transmission and emission scans are performed using a ${ }^{137} \mathrm{Cs}$ source [12].

After acquisition of the dynamic data in sinogram form and correction for the above effects, image reconstruction must be executed to form tomographic images of the volume of interest. Reconstruction is performed using analytic methods, such as filtered backprojection (FBP), or iterative techniques, including maximum likelihood expectation maximization (MLEM) and ordered subset expectation maximization (OSEM). The results of this process are dynamic images of the tracer distribution in the detector plane.

At the UOHI, PET data are acquired in $3 \mathrm{D}$ mode and subsequently rebinned into equivalent 2D slices. Photon attenuation, scatter, randoms, and dead-time corrections are applied using the ECAT v7.2 software. FBP with a $12 \mathrm{~mm}$ Hann window of the ramp filter is then typically used to perform image reconstruction on each independent 2D plane. After correcting for isotope decay, images are calibrated to yield tracer concentration in $\mathrm{Bq} / \mathrm{cc}$.

\subsection{Cardiac Image Processing}

Following application of the appropriate corrections and image reconstruction, measured PET values in the heart can be obtained from the resultant images. At the UOHI, processing is completed using an in-house developed FlowQuantC package. With this software, the LV is located in the FOV by fitting partial ellipses to the data in three 
orthogonal planes: transaxial, vertical long axis (VLA), and horizontal long axis (HLA) (Fig. 1.2) [13]. Following this step, automatic reorientation of the images into short-axis (SA) sections, taken perpendicular to the long-axis plane, is performed (Fig. 1.3). An image of the tracer uptake in the myocardium is formed from the mean of the last few time frames. Maximum myocardial activity profiles are then subsequently obtained by sampling the uptake image in a combined conical and planar coordinate system, and are utilized to define the 3-dimensional shape of the LV (Fig. 1.4). The resulting set of midmyocardial coordinates is used to resample the short-axis images into 2-dimensional dynamic polar maps (Fig. 1.5). Polar maps are a circular 2D representation of the LV myocardium, where the centre depicts the apex and the outermost ring represents the base. From the polar maps, the measured myocardial time activity curves (TACs) for each sector of the myocardium, $C_{\text {meas }}(t)$, can be obtained. The arterial blood time-activity curve, $C_{a}(t)$, is obtained from the average of 12 short-axis image regions placed automatically in the center of the cavity and base regions of the LV and in the center of the left atrium (Fig 1.3). Typical blood and myocardial TACs are shown in Figure 1.6.

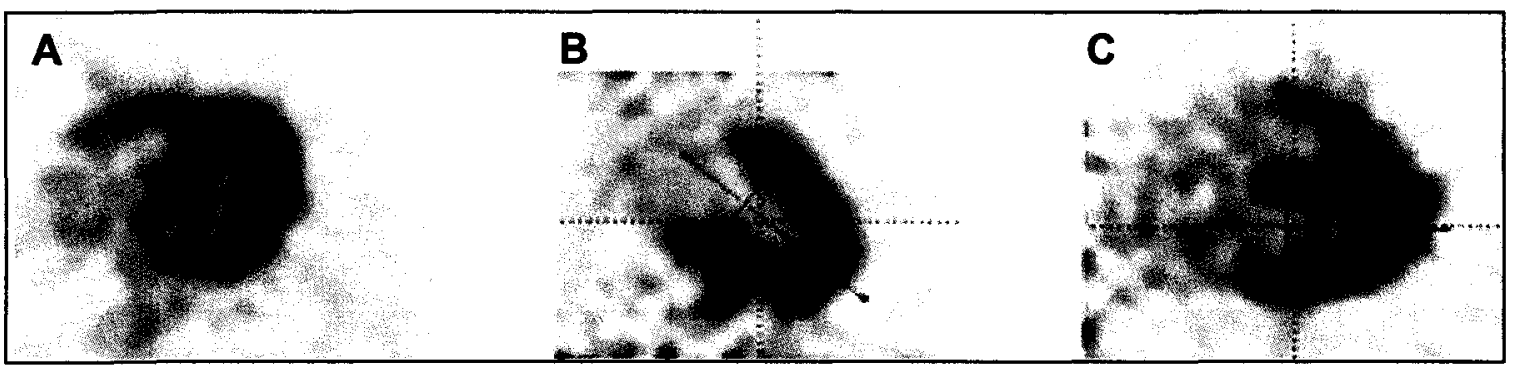

Figure 1.2: (A) Transaxial, (B) VLA, and (C) HLA slices of the myocardium. The LV was located in the FOV by fitting partial ellipses to the images in the three orthogonal planes. 


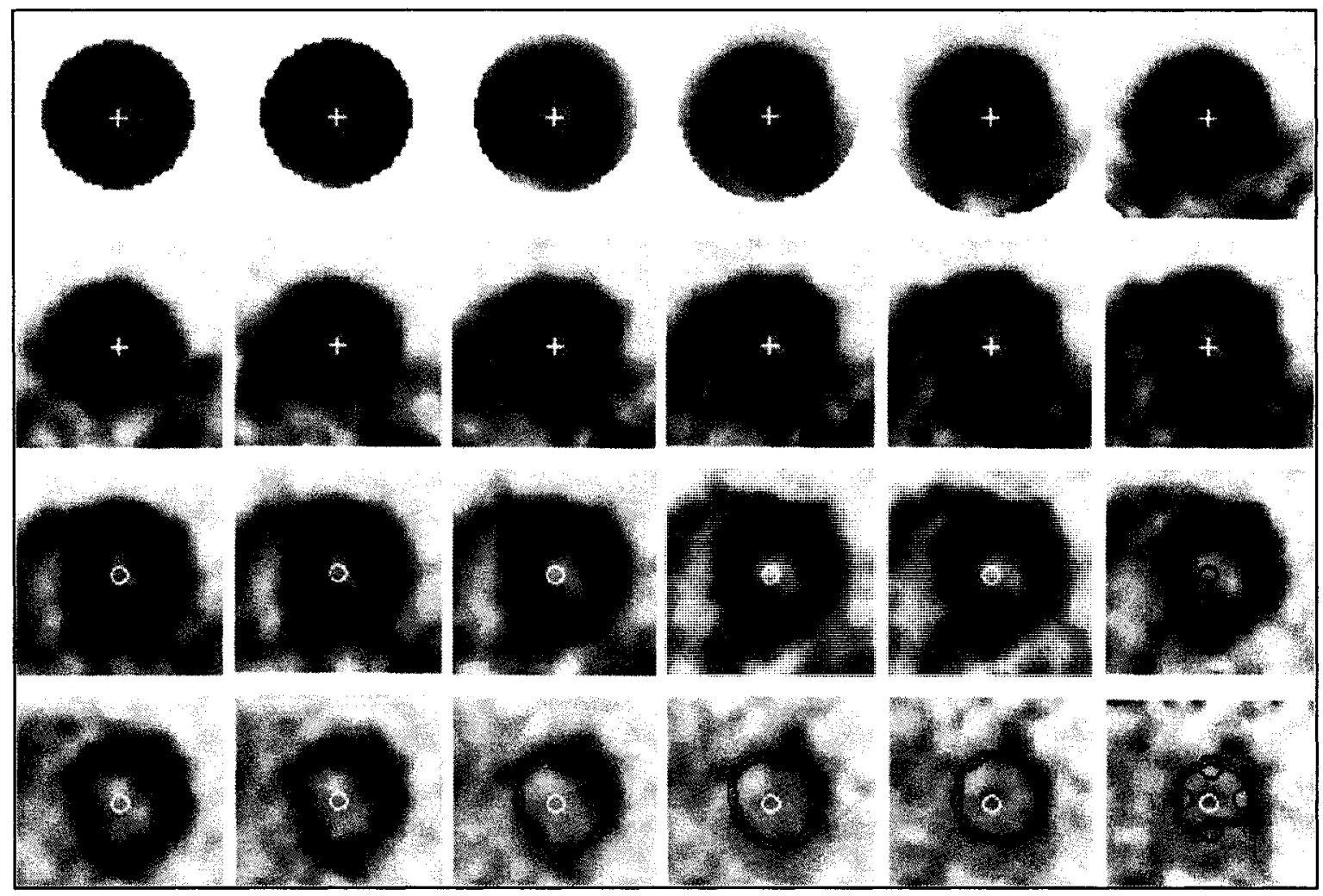

Figure 1.3: Sampling of the SA slices. The myocardium was sampled to define the activity in the tissue. The arterial blood TAC was obtained by averaging the last 12 SA image regions placed automatically in the center of the cavity and base regions of the $L V$ and in the center of the left atrium, as shown by the white circles in the center of the SA slices.
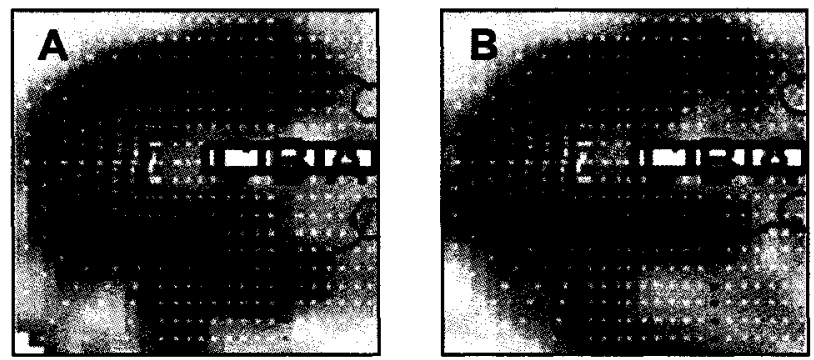

Figure 1.4: Sampling of the (A) VLA and (B) HLA. The dotted white lines indicate the planes along which the $S A$ slices were sampled. At the apex, conical sampling was used as it better approximates the shape of the $L V$ in that region, whereas planar sampling was used for the remainder of the $\mathrm{LV}$. The blood pool activity is sampled in the cavity $(C)$, base $(B)$, and atrium $(A)$ regions. 

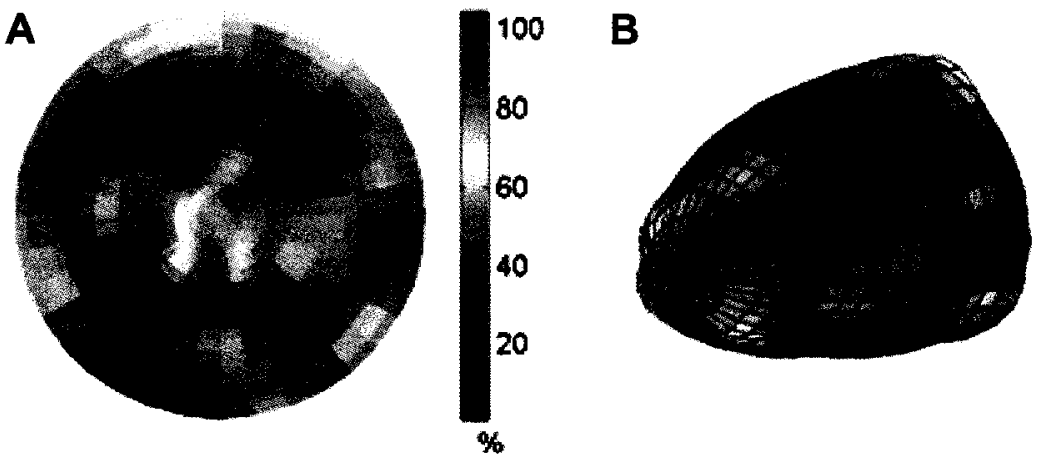

Figure 1.5: (A) 2D representation of the $\mathrm{LV}$ in polar map format. The centre represents the apex and the outermost ring represents the base. The colours are representative of the uptake (\%) of the tracer in the myocardial tissue, where red indicates maximal uptake and blue indicates the absence of uptake. (B) $3 D$ view of the data that is presented in the polar map.

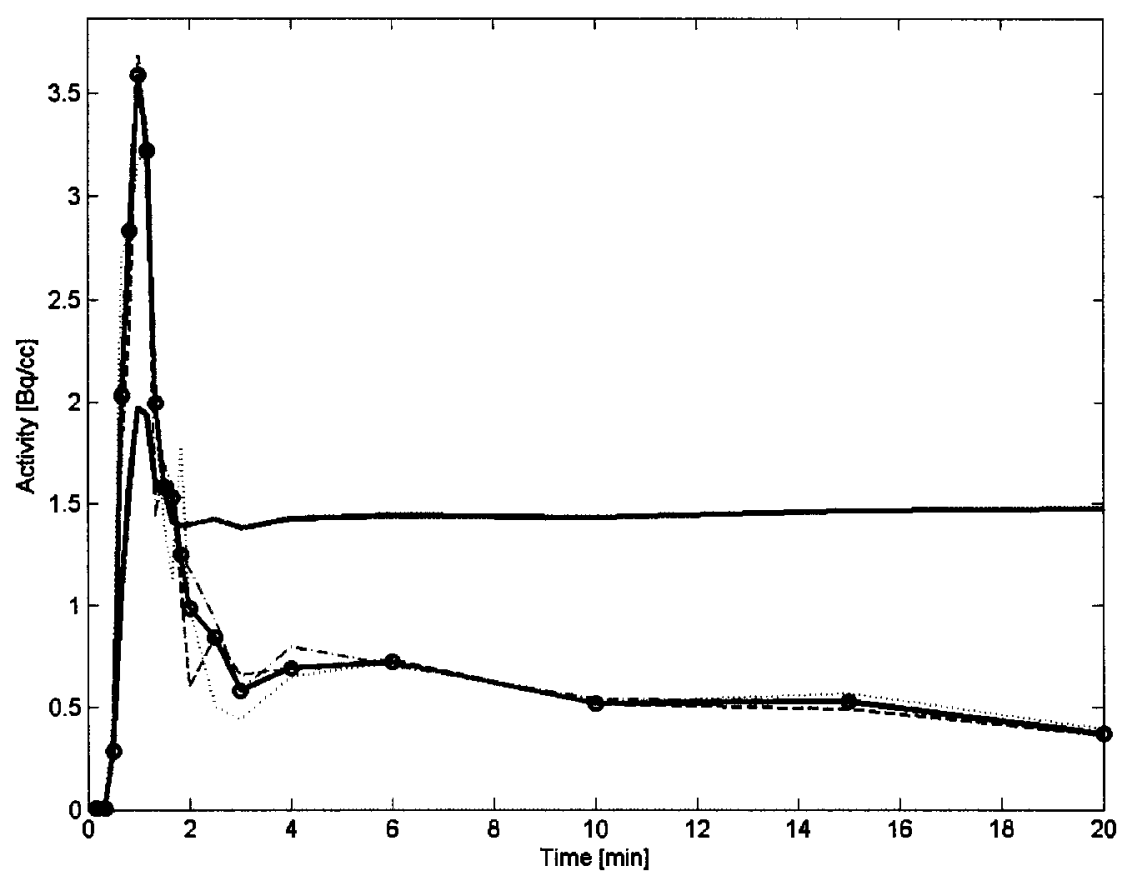

Figure 1.6: Blood and myocardial TACs. The solid red line represents the overall blood TAC, which is the median of the TACs from the cavity (magenta dashed line), base (cyan dashed line) and atrium (green dotted line) regions. The solid blue line is the measured myocardial TAC. 
In reality, the measured myocardial TAC, $C_{\text {meas }}(t)$, is not purely the signal measured from the myocardial tissue. In an ideal scenario, the intensity of each pixel in a PET image would be proportional to the concentration of activity corresponding to that particular volume of tissue within the subject. However, due to the finite spatial resolution of the PET scanner and organ motion due to the respiratory and cardiac cycles, the PET images are blurred, leading to a mixing of activity from adjacent pixels. This effect is known as partial volume (PV) averaging [14].

The effects of PV averaging are divided into two categories: spillover and PV losses. Spillover occurs when the target pixels in the region of interest (ROI) have lower activity than the pixels in the surrounding regions and the activity from those regions appears to "spill over", or blur, into the ROI. This leads to an overestimation of the true activity within the ROI. However, if the converse occurs and the activity from the ROI blurs into the pixels of adjacent regions, this effect is referred to as PV loss. PV losses result in an underestimation of the true activity in the ROI. This effect manifests itself when the object of interest is less than twice the size of the reconstructed image resolution.

Both of the PV averaging effects are problems for cardiac PET imaging of the myocardium. Typically, activity from the arterial blood pool in the cavity of the LV spills over onto the myocardium in a PET image, resulting in an overestimation of activity, particularly in the first-pass transit through the heart. Additionally, the thickness of the LV wall is usually on the order of $8-12 \mathrm{~mm}$, while a typical clinical reconstructed image has a resolution in the range of $10-15 \mathrm{~mm}$, resulting in an underestimation of the true 
myocardial activity. An example of these effects for the activity in a $10 \mathrm{~mm}$ thick myocardial wall and $10 \mathrm{~mm}$ scanner resolution is shown in Figure 1.7. These problems are compounded by the facts that the myocardial wall thickness is not constant over the entire LV, and some parts of the myocardium are more susceptible to motion than others. This is typical of the apex, which is thinner and undergoes more motion than the outermost parts of the LV, resulting in its activity being commonly underestimated (Fig. 1.8). If left uncorrected, these effects can lead to inaccurate quantification of the processes of interest in cardiac PET imaging, such as MBF, potentially leading to misdiagnosis of disease in patients [15].

To correct for PV averaging, a recovery coefficient (RC) can be used. RC is expressed as the ratio of the measured activity to the true activity, as shown in Figure 1.7. In effect it quantifies the PV losses. Its value can be estimated for different regions of the myocardium using tracer kinetic modeling, or a fixed value for RC can be assumed. Once determined, the parameter of interest is corrected by this factor to obtain its true value. This concept is further discussed in the context of kinetic modeling in Chapter 2, section 2.2 and Chapter 3, section 3.4. From the TACs obtained from the processed images, tracer kinetic modeling techniques can be applied to quantify the biological parameters of interest, such as $\mathrm{MBF}$ and flow reserve, which are discussed in the subsequent sections. 


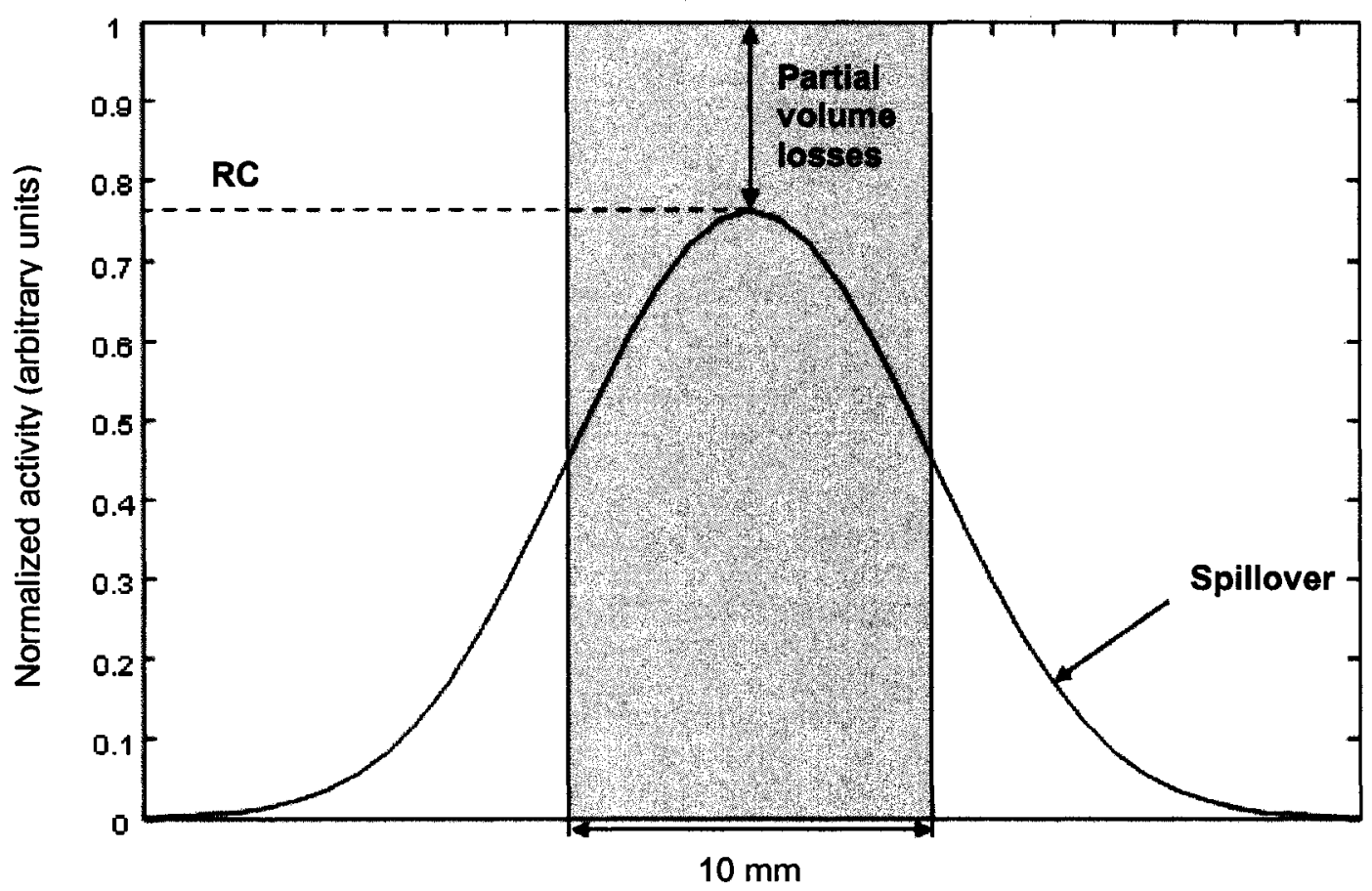

Figure 1.7: $\mathrm{PV}$ averaging in a simulated $10 \mathrm{~mm}$ thick uniform myocardial wall with $10 \mathrm{~mm}$ scanner resolution. The line profile shows that the peak activity in the wall is underestimated by $24 \%(\mathrm{RC}=0.76)$.

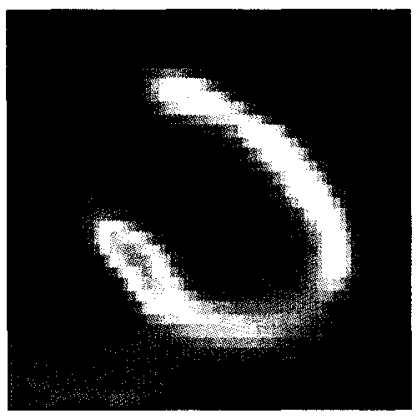

Figure 1.8: ${ }^{82} \mathrm{Rb}$ VLA image of the LV from a normal subject. In a healthy subject, uniform uptake should be observed in the entire myocardial wall; however, due to PV effects at the apex, it appears that the activity is reduced (black arrow). In patients, this could lead to a misdiagnosis of disease. 


\subsection{Myocardial Blood Flow and Flow Reserve}

Determination of regional $\mathrm{MBF}$, or myocardial perfusion, in humans, is an important prognostic marker for patients with heart disease. MBF can be determined on both relative and absolute scales. On a relative scale, MBF is represented qualitatively as a measure of the tracer uptake into the tissue. In absolute terms, MBF is a measure of the quantity of blood supplied to a given volume of tissue per unit time, and is expressed in units of $\mathrm{mL} / \mathrm{min} / \mathrm{g}$. By obtaining relative or absolute estimates of blood flow in the resting state and under exercise conditions, myocardial flow reserve (MFR) can be obtained. MFR is defined as the ratio of maximal blood flow/resting flow in the LV myocardium. Assessment of myocardial perfusion and flow reserve can be performed using PET with a number of tracers, as described in the subsequent section.

\subsection{PET Tracers for Blood Flow Imaging}

For cardiac applications, tracers can be used to investigate blood flow to the myocardium, as well as myocardial viability and receptor function. The most common cardiac PET tracers and their uses are listed in Table 1.2. The majority of these PET tracers are produced in a cyclotron by bombarding a specific target nucleus with a beam of high energy protons, transforming the initial nucleus into a proton rich isotope. In many cases, the isotope must then undergo additional chemical reactions to be synthesized into a tracer molecule. As this process is complex and expensive, with the short tracer half-lives requiring each scanning facility to have an on-site cyclotron, there has been a shift 
towards developing simpler and more cost-effective alternatives, particularly for blood flow imaging.

Table 1.2: Commonly used PET tracers [16]

\begin{tabular}{l|c|c|c}
\hline Tracer & $\begin{array}{c}\text { Isotope Half- } \\
\text { life }(\mathrm{min})\end{array}$ & Application & $\begin{array}{c}\text { Mode of } \\
\text { Production }\end{array}$ \\
\hline${ }^{18} \mathrm{~F}$-FDG & 109.8 & Metabolism & Cyclotron \\
${ }^{11} \mathrm{C}$-acetate & 20.4 & Metabolism & Cyclotron \\
${ }^{13} \mathrm{NH}_{3}$ & 9.97 & Perfusion & Cyclotron \\
${ }^{15} \mathrm{O}$-water & 2.03 & Perfusion & Cyclotron \\
${ }^{82} \mathrm{Rb}$ & 1.27 & Perfusion & Generator \\
\hline
\end{tabular}

For imaging blood flow, the ideal tracer would be one that is freely diffusible across the capillary and cell membranes and fully extracted in proportion to blood flow from the arterial blood pool into the tissue on the first pass through the heart. Additionally, such a tracer should be physiologically retained in the tissue once taken up, and have a long enough half-life to permit production and subsequent imaging. Practically however, none of the current tracers that are used for blood flow imaging satisfy all of these criteria [17].

The clinical standard tracer for quantitative flow imaging is ${ }^{13} \mathrm{NH}_{3}$. With a relatively long half-life of 10 minutes, this freely diffusible tracer passively crosses the capillary and cell membranes to enter the tissue, being nearly fully extracted on the first transit through the circulation in proportion to blood flow at low flow rates, and is subsequently retained in the tissue by conversion into ${ }^{13} \mathrm{~N}$-glutamine. However, the net retention of the tracer in the tissue decreases as flow rates increase [17], and the cost of producing ${ }^{13} \mathrm{NH}_{3}$ is quite substantial. A cost-effective alternative for blood flow imaging that is the focus of this 
research is the generator-produced tracer ${ }^{82} \mathrm{Rb}$. This tracer is the clinical standard for qualitative, or relative, myocardial perfusion imaging, with PET.

${ }^{82} \mathrm{Rb}$ is a first column element in the periodic table that can act as a potassium analog. The rubidium cation $\left(\mathrm{Rb}^{+}\right)$can replace the potassium cation $\left(\mathrm{K}^{+}\right)$in the sodium-potassium ion exchange system and thus be actively transported across the cell membrane and into the tissue, where it is normally retained. ${ }^{82} \mathrm{Rb}$ is produced using a ${ }^{82} \mathrm{Sr} /{ }^{82} \mathrm{Rb}$ generator [17]. With a half-life of 25.5 days, ${ }^{82} \mathrm{Sr}$ can be used continuously over a period of approximately 8 to 10 weeks to produce ${ }^{82} \mathrm{Rb}$ by decay. Once formed, ${ }^{82} \mathrm{Rb}$ can be directly infused into the patient as ${ }^{82} \mathrm{RbCl}$ for imaging through the advent of generator infusion systems [18]. With a relatively short half-life of 76 seconds, this permits rapid turnaround between successive scans in the same patient as the tracer quickly decays into stable krypton $\left({ }^{82} \mathrm{Kr}\right)$. This also minimizes the radiation exposure to the patient. However, ${ }^{82} \mathrm{Rb}$ is not as highly extracted on the first pass transit through the circulation as ${ }^{13} \mathrm{NH}_{3}$, making it a less than ideal blood flow tracer [19]. Yet, this tracer is more practical and economical. The development of this mobile alternative is permitting centres that would otherwise be unable to assess blood flow due to their lack of a cyclotron, to provide more thorough non-invasive diagnoses to patients. Relative and absolute methods used for imaging of MBF and MFR with ${ }^{82} \mathrm{Rb}$ and ${ }^{13} \mathrm{NH}_{3}$ are discussed in the following sections. 


\subsection{Relative Myocardial Perfusion Imaging with PET}

Relative perfusion imaging is an accurate method for identifying perfusion defects in patients in whom a region of the heart with relatively normal myocardial perfusion exists and can be used for qualitative, comparative measures of net uptake and clearance of the tracer. Relative perfusion imaging with ${ }^{82} \mathrm{Rb}$ has been demonstrated for the accurate detection and localization of single-vessel coronary artery disease (CAD) with PET most recently by Bateman et al. [20]. However, patients lacking a truly normal region, such as those with disease in all three major coronary vessels, may be incorrectly evaluated or misdiagnosed. In these cases, quantification of absolute MBF may be advantageous.

\subsection{Absolute Myocardial Blood Flow Imaging with PET}

Absolute, or quantitative, perfusion imaging is performed using mathematically and physiologically appropriate models to describe the biological behaviour of the administered radiotracers in myocardial tissue over time. It has been shown in previous work that quantification of myocardial perfusion with dynamic PET imaging may improve the detection of severe or balanced multi-vessel CAD [21,22]. Absolute flow imaging with ${ }^{82} \mathrm{Rb}$ can also be used to measure MFR which may further aid in the functional assessment of coronary artery stenoses (narrowings) [23,24]. MFR measurements are also effective in defining the extent of microvascular dysfunction in various cardiomyopathies, and may aid in the early detection of atherosclerotic disease in asymptomatic patients with coronary risk factors such as diabetes, hyperlipidemia, 
hypertension and smoking, as described in recent reviews by Kaufmann et al. and deKemp et al. [16,25].

The cyclotron-produced tracers ${ }^{15} \mathrm{O}$-water and ${ }^{13} \mathrm{NH}_{3}$ are widely accepted for the accurate quantification of absolute MBF and MFR with PET. ${ }^{13} \mathrm{NH}_{3}$ is used clinically for relative MPI [26,27], as well as absolute flow quantification via compartmental modeling $[28,29,30,31]$. Compartmental models of ${ }^{13} \mathrm{NH}_{3}$ have been validated in human studies, most recently by Nitzsche et al. [32]. In PET centers without a cyclotron, the generatorproduced tracer ${ }^{82} \mathrm{Rb}$ is used increasingly to image relative myocardial perfusion, as discussed in a review by Di Carli et al. [19]. Quantification of myocardial blood flow and MFR using ${ }^{82} \mathrm{Rb}$ PET and compartmental modeling has also been validated in animals $[33,34]$ and most recently in human studies using a one-compartment model by Lortie et al. [35], and a two-compartment model by Lin et al. [36]. While compartmental models are commonly used for flow quantification, they are relatively complex as compared to a simpler retention model, which is a robust method that may allow for more routine clinical quantification of flow and MFR [24]. As demonstrated by Choi et al. [31] and Yoshida and colleagues [24], the net retention method is computationally simple and can generate precise flow estimates with both ${ }^{13} \mathrm{NH}_{3}$ and ${ }^{82} \mathrm{Rb}$, in normal myocardium.

Recent work by Santana et al. has demonstrated that stress ${ }^{82} \mathrm{Rb}$ PET images of relative myocardial perfusion can be compared with a database defining the regional lower limit of normal to aid in the diagnosis and localization of CAD [37]. However, similar diagnostic databases defining the normal range of absolute MBF and MFR have only 
been reported using ${ }^{13} \mathrm{NH}_{3}$ PET and compartmental modeling [21,22]. The analysis methods used in the present study to obtain measurements of MBF and MFR, and to form normal population databases with ${ }^{82} \mathrm{Rb}$ are discussed in the remaining chapters.

\subsection{Thesis Overview}

Chapter 2 describes the technique of kinetic modeling for processed dynamic PET images and explores the concept of tracer extraction, including a description of the common compartmental models used to describe the kinetics of ${ }^{13} \mathrm{NH}_{3}$ and ${ }^{82} \mathrm{Rb}$. The development of a simplified net retention model for the analysis of the biological behaviour of the radiotracers in tissue over time is explored in Chapter 3. The work presented in section 3.4 on the development of extraction corrections for the net retention model was presented orally and published as an abstract for the 2008 Society of Nuclear Medicine Annual Meeting [2]. Chapter 4 examines the purpose of normal population databases and the use of the net retention model as well as the compartmental models for the formation of such databases. The results from section 4.3 were presented as a poster and published in a short manuscript in the conference proceedings for the 2007 IEEE Nuclear Science Symposium and Medical Imaging Conference [1]. The subsequent use of the normal population databases to detect and localize disease in patients is addressed in Chapter 5. This includes an analysis of the receiver operator characteristics, sensitivity, and specificity of the databases. The concluding chapter summarizes the findings of this research project and discusses potential future work. 


\section{CHAPTER 2 - Tracer Kinetic Modeling}

\subsection{Principles of Kinetic Modeling}

Dynamic PET studies provide a measure of the activity of an injected radiotracer in the body over time. By acquiring this data, information about the behaviour of the tracer in the system can be determined and further used to assess specific biological functions. To quantify this dynamic process, tracer kinetic modeling is used.

Tracer kinetic modeling describes the time-varying distribution of a radiotracer in the body by imposing a mathematical model on the biological system. Most applications of tracer kinetic principles are based on compartment models. Compartmental modeling is a mathematical technique that represents systems as a collection of a finite number of compartments containing varying amounts of radioactive tracer. The model requires input of the TAC of the tracer, which can be determined by sampling the arterial blood cavity regions from the measured PET data, as previously described in section 1.5. The output of the model is the TAC of the tissue of interest, in this case the myocardium. A compartment is defined as a volume wherein the tracer becomes homogeneously distributed at a rate that is assumed to be instantaneous, or more practically at a rate that is at least more rapid than the rate of tracer exchange between compartments. Each compartment is a representation of a separate physical space within the system, such as 
the vascular versus the extravascular tissue space, or a unique chemical form or pharmacological state of the tracer, such as bound versus unbound receptor ligand [10].

Compartments are linked to adjacent spaces by kinetic processes, providing pathways for tracer exchange. Tracer kinetics describe the speed at which the tracer is transferred between compartments using a set of rate constants. In cases where two compartments represent different physical spaces, the rate constants linking them are representative of flow or transport across a physical boundary, whereas the rate constants represent transformation rates in cases where the adjoining compartments are different chemical or pharmacological states. These parameters form the basis of first-order differential equations, which follow the law of conservation of mass, and describe the concentration of the radiotracer in the compartments as a function of time. A generalized compartment model is shown in Figure 2.1 [38].

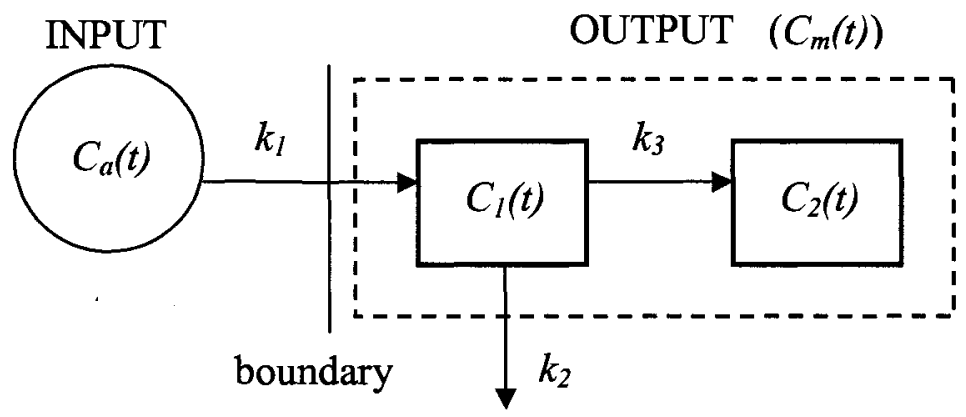

Figure 2.1: A generalized two-compartment model, including two distinct compartments wherein, the tracer concentration is denoted by $C_{i}(t)$, and three rate parameters, $k_{i}$, describing the rate of tracer exchange between adjoining compartments [38].

This depiction consists of two distinct compartments linked by three rate parameters. The arrows that point to the right indicate influx of tracer into the compartments, whereas the 
down-pointing arrow represents efflux, in units of inverse time $\left(\mathrm{min}^{-1}\right)$. The input to the model is the arterial blood TAC, $C_{a}(t)$, and the output is the myocardial TAC, $C_{m}(t)$, which is the sum of the tracer concentration in the first and second compartments. The differential equations that describe the rate of tracer exchange between the compartments can be expressed as follows:

$$
\begin{aligned}
& \frac{d C_{1}(t)}{d t}=k_{1} C_{a}(t)-k_{2} C_{1}(t)-k_{3} C_{1}(t) \\
& \frac{d C_{2}(t)}{d t}=k_{3} C_{1}(t)
\end{aligned}
$$

where $d C_{i}(t) / d t$ represents the rate of change of tracer concentration in compartment $i$; $C_{1}(t)$ and $C_{2}(t)$ represent the concentration of tracer in the first and second compartments, respectively. The solutions to first-order linear differential equations of this form are expressed as exponentials convolved with the input function. As there are two compartments in this case, the general solution is a biexponential curve. However, when there is no efflux of tracer from the second compartment, as is the case in this example, the expression simplifies to the following [28]:

$$
C_{m}(t)=C_{1}(t)+C_{2}(t)=\frac{K_{1} k_{2}}{k_{2}+k_{3}} e^{-\left(k_{2}+k_{3}\right) t} \otimes C_{a}(t)+\frac{K_{1} k_{3}}{k_{2}+k_{3}} \int_{0}^{t} C_{a}(\tau) d \tau
$$

where $\otimes$ represents the 1 -dimensional convolution operator. By fitting this model to the measured tissue TAC, the values of the rate constants can be determined using a nonlinear regression technique. Quantitative representations of specific biologic processes, including blood flow, can then be obtained either directly from the parameter estimates or through subsequent relationships between the parameters and other factors, such as tracer extraction [38]. 


\subsection{Tracer Extraction}

A tracer is exchanged between the blood and tissue space via diffusion or active transport across the physical boundary of the capillary wall. While tracer uptake from the blood pool into the tissue is dependent on blood flow, it is also governed by the extraction of the tracer into the tissue. The first-pass unidirectional tracer extraction fraction is defined as the amount of tracer extracted from the blood, through the capillaries and into the tissue during the initial transit of the tracer through the circulation, without consideration of back-transfer from the tissue into the blood. Since tracer extraction occurs through capillary membranes, the extraction fraction depends on the surface area of the capillaries, $S$, the permeability of the capillaries to the tracer, $P$, and the blood flow through the capillaries into the tissue (myocardium), $M B F . P$ and $S$ form the permeability-surface area product $(P S)$ which is unique to a given tracer $[10,39,40]$. A simple model relating these quantities is the Renkin-Crone model, whereby the capillary is represented as a rigid tube (Fig. 2.2). According to this model, the unidirectional tracer extraction, $E$, is represented as:

$E(M B F)=1-e^{-P S / M B F}$

However, experimentally measured extraction fractions have been shown to be larger at higher flow values than this equation predicts, indicating that the PS product is not constant but rather increases with increasing flow due to increased capillary recruitment at high flow. Thus, the model was modified to reflect this dependence as follows [17]:

$$
E(M B F)=1-e^{-(A+B \cdot M B F) / M B F}
$$


where $(A+B \cdot M B F)$ is the flow-dependent $P S$ product, and $A$ and $B$ can be experimentally obtained for a particular tracer by fitting this relationship to measured uptake rate data. The tracer uptake is then represented as the product of the unidirectional (first-pass) tracer extraction and blood flow.

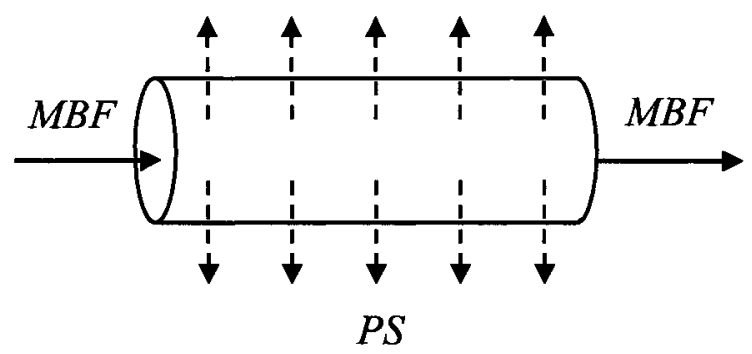

Figure 2.2: Renkin-Crone capillary model of tracer extraction. The capillary is assumed to be a rigid tube with the extraction of tracer from blood to tissue described by the permeability-surface area product (PS), and the blood flow through the capillary is represented as $M B F[10]$.

For most of the physiologically retained tracers used to measure blood flow, the extraction fraction is less than unity and decreases with increasing flow due to the reduced time for tracer exchange to occur across the membrane at high flow rates. As shown in Figure 2.3, while an ideal, freely diffusible perfusion tracer, such as ${ }^{15} \mathrm{O}$-water, is nearly $100 \%$ extracted at all flow ranges, ${ }^{13} \mathrm{NH}_{3}$ is also considered to be essentially $100 \%$ extracted except at high flows, which are generally outside the physiologically relevant range; however, ${ }^{82} \mathrm{Rb}$ demonstrates a substantial decline in flow-dependent extraction at higher flow values. Therefore, in order to obtain accurate flow estimates for such tracers using a compartment model, the uptake rate of the tracer must be corrected for tracer extraction. This is accomplished by including the tracer-specific extraction fraction, $E$, in the kinetic model of tracer uptake. This concept is further addressed in the 
subsequent sections, which discuss the compartmental models used for ${ }^{13} \mathrm{NH}_{3}$ and ${ }^{82} \mathrm{Rb}$ cardiac PET imaging, with a focus on those used specifically in this research project.

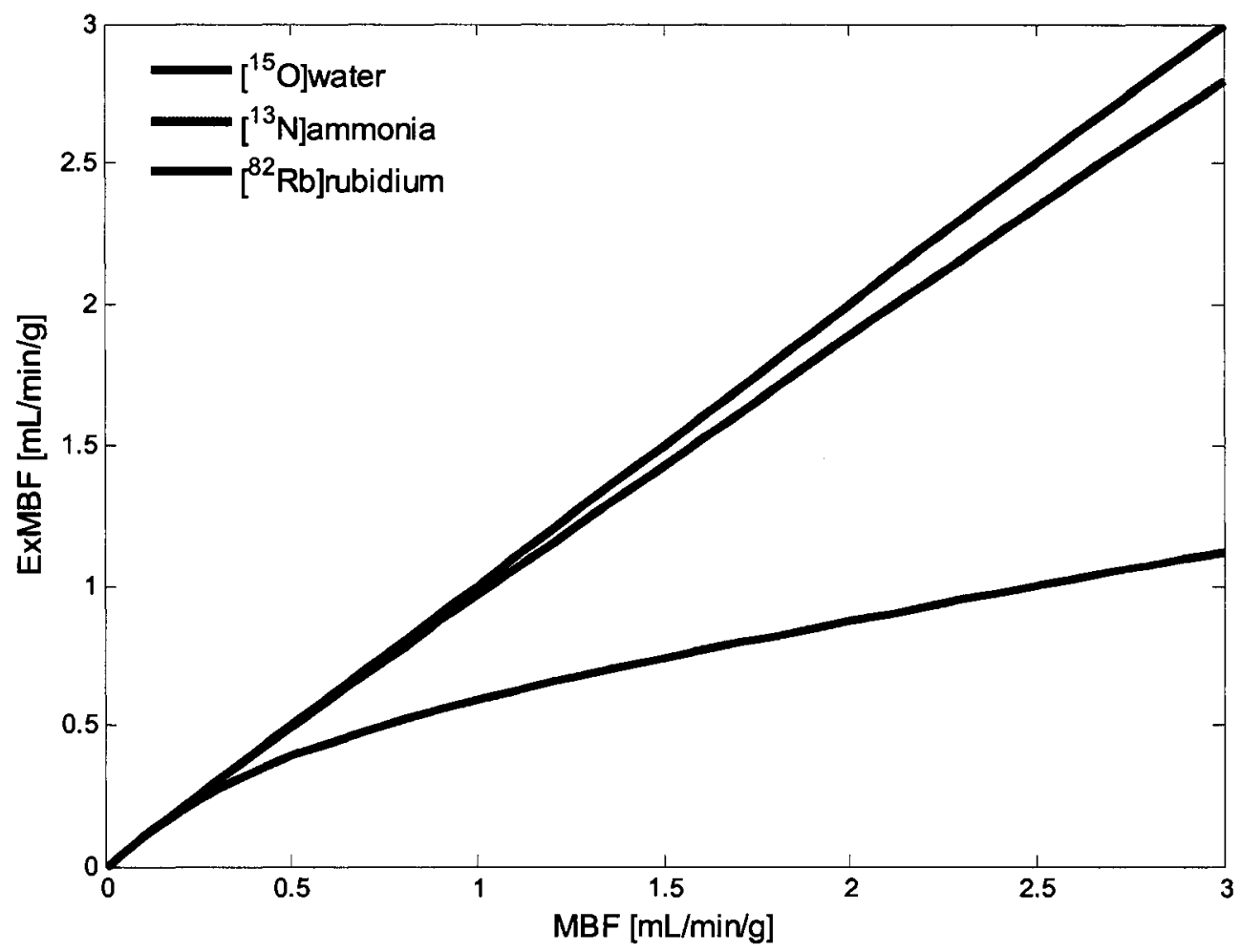

Figure 2.3: Uptake rate of flow tracers in the myocardium as a function of MBF. The uptake is the product of the unidirectional (first-pass) extraction and MBF. Note that the extraction of ${ }^{82} \mathrm{Rb}$ declines noticeably at high flow rates, whereas that of the ideal blood flow tracer, ${ }^{15} \mathrm{O}$-water, remains consistent at unity, and the extraction of ${ }^{13} \mathrm{NH}_{3}$ drops off slightly at high flow values (adapted from [16]).

\subsection{Two-Compartment Models}

Two-Compartment Models for ${ }^{13} \mathrm{NH}_{3}$

The kinetic properties of ${ }^{13} \mathrm{NH}_{3}$ in the myocardium are commonly described using a 2compartment model, as depicted in Figure 2.4 [28]. This method gives a complete 
physiological description of the kinetics of ${ }^{13} \mathrm{NH}_{3}$ in the body. The input to the model is the concentration of ${ }^{13} \mathrm{NH}_{3}$ in the arterial blood, $C_{a}(t)$. Since ${ }^{13} \mathrm{NH}_{3}$ is rapidly metabolized in the liver into the molecular by-products of urea and glutamine, the arterial blood activity is actually a combination of ${ }^{13} \mathrm{NH}_{3}$ and its metabolites. Therefore, to obtain a correct estimate of the model parameters, the arterial blood component must be corrected for the presence of ammonia metabolites in the system. A metabolite correction of the form:

$$
C_{\hat{\mathrm{a}}}(t)=e^{-\ln (2) \cdot(t-0.48) / 6.69} \times C_{a}(t)
$$

can be applied to the measured blood curve $C_{a}(t)$ to obtain $C_{\hat{a}}(t)[41,42]$.

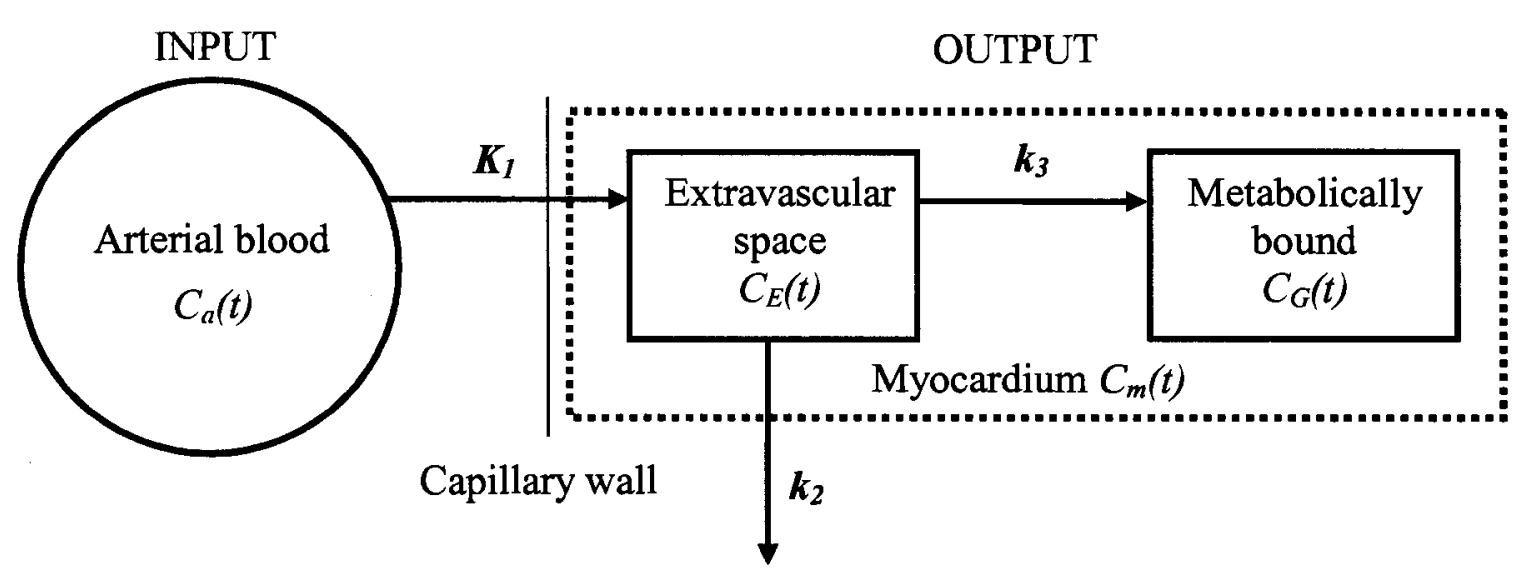

Figure 2.4: Two-compartment model describing the uptake and retention of ${ }^{13} \mathrm{NH}_{3}$ in myocardial tissue. $K_{1}$ represents the exchange of ammonia between the arterial blood and the extravascular space. $k_{2}$ reflects the washout of ammonia from the extravascular space, and $k_{3}$ describes the rate at which ${ }^{13} \mathrm{NH}_{3}$ is metabolized into glutamine [28].

The first and second compartments in the model comprise the total concentration of ${ }^{13} \mathrm{NH}_{3}$ in the myocardial tissue, $C_{m}(t)$. This volume is further broken down into the 
extravascular space, $C_{E}(t)$ and the metabolically bound space, $C_{G}(t)$. The extravascular space represents the concentration of intracellular and extracellular ${ }^{13} \mathrm{NH}_{3}$ in the myocardial tissue of the heart, while the metabolic space describes the intracellular concentration of metabolized ${ }^{13} \mathrm{NH}_{3}$ (glutamine) in the myocardium. The parameters $K_{l}$, $k_{2}$, and $k_{3}$ are rate constants describing the tracer movement between compartments. $K_{l}$ expresses the rate of ${ }^{13} \mathrm{NH}_{3}$ uptake into the tissue ( $\mathrm{mL}$ blood $/ \mathrm{g}$ tissue/min), and serves as a direct estimate of MBF since the unidirectional extraction of the tracer across the capillary membrane is near unity over a wide flow range [27]. The parameter $k_{2}$ describes the rate of ammonia washout from the tissue $\left(\mathrm{min}^{-1}\right)$, and $k_{3}$ characterizes the rate at which metabolized ${ }^{13} \mathrm{~N}$-glutamine is formed $\left(\mathrm{min}^{-1}\right)$ [43]. Note that $K_{I}$ is denoted with an uppercase $K$ as the units of this parameter are different than the other rate constant units of $\min ^{-1}$.

The model compartments and rate constants can be characterized by a set of first order differential equations:

$$
\begin{aligned}
& \frac{d C_{E}(t)}{d t}=K_{1} C_{a}(t)-k_{2} C_{E}(t)-k_{3} C_{E}(t) \\
& \frac{d C_{G}(t)}{d t}=k_{3} C_{E}(t)
\end{aligned}
$$

This system describes the rate of change of ${ }^{13} \mathrm{NH}_{3}$ concentration in the myocardial tissue over time for each compartment of the model. The solution of these equations describes $C_{m}(t)$, the concentration of ${ }^{13} \mathrm{NH}_{3}$ in the myocardial tissue as a function of time [28], and is expressed in Eq. 2.3, with the exception that $K_{I}$ is capitalized in this case. 
To account for the issues of spillover from the blood pool and partial-volume recovery, the following geometric model is used:

$$
C_{\text {meas }}(t)=F_{a} C_{a}(t)+\left(1-F_{a}\right) C_{m}(t)
$$

where $C_{\text {meas }}(t)$ is the measured myocardial tracer concentration, $F_{a}$ is the fractional blood volume spillover and $\left(1-F_{a}\right)$ is an equivalent estimate of $\mathrm{RC}$, thus it corrects for regional partial-volume loss in the myocardium [15]. By fitting these equations to the blood and myocardial tissue TACs, the model parameters $\left(K_{l}, k_{2}, k_{3}, F_{a}\right)$ are estimated using a weighted least squares method [44] with weights equal to the square-root of the frame lengths. This method ultimately provides a direct quantitative representation of perfusion $\left(K_{I}\right)$, which is the primary parameter for the assessment of disease severity in cardiac studies.

A variation of this model is the constrained method, where $k_{2}$ is set to a constant fraction of $K_{l}$. This stabilizes the values in the low-flow regions by reducing the number of parameters to estimate simultaneously [45]; however this results in a trade-off in the precision versus the accuracy of the parameter estimates and as such this model is not commonly utilized.

An alternate two-compartment model that can be used for the evaluation of MBF with ${ }^{13} \mathrm{NH}_{3}$ [30], is depicted in Figure 2.5. In this model, the freely diffusible ${ }^{13} \mathrm{NH}_{3}$ in the vascular and extravascular spaces is represented as one compartment (freely diffusible space), and the second compartment represents the same metabolically-trapped ${ }^{13} \mathrm{~N}$ glutamine (trapped space). However, unlike the previously described two-compartment 
model, the rate constants, $K_{1}$ and $k_{2}$, represent the influx and efflux of tracer between the two compartments, rather than between the arterial blood and the tissue compartments. The concentrations of tracer in the freely diffusible and metabolically-bound compartments are denoted by $C_{F}(t)$ and $C_{T}(t)$, respectively. $C_{a}(t)$ is the arterial blood pool tracer concentration $(\mathrm{Bq} / \mathrm{cc})$ and $D V$ is the volume of distribution for ${ }^{13} \mathrm{NH}_{3}(\mathrm{~mL} / \mathrm{g}) . D V$ is the ratio of the tissue and blood tracer concentrations when the concentrations have reached a state of equilibrium, i.e. when the net tracer flux between the two compartments is zero. While the name may seem to be a misnomer as it is stated to represent a ratio of concentrations, it is called a volume as it is equivalent to the volume of blood that contains the same activity as $1 \mathrm{~g}$ of tissue. Furthermore, since the net exchange of tracer is zero at equilibrium, $D V$ can also be expressed as:

$D V=\frac{K_{1}}{k_{2}}$

This parameter is useful since it is often easier to solve for $D V$ in the model fitting algorithm rather than attempting to estimate its individual component parameters [46].

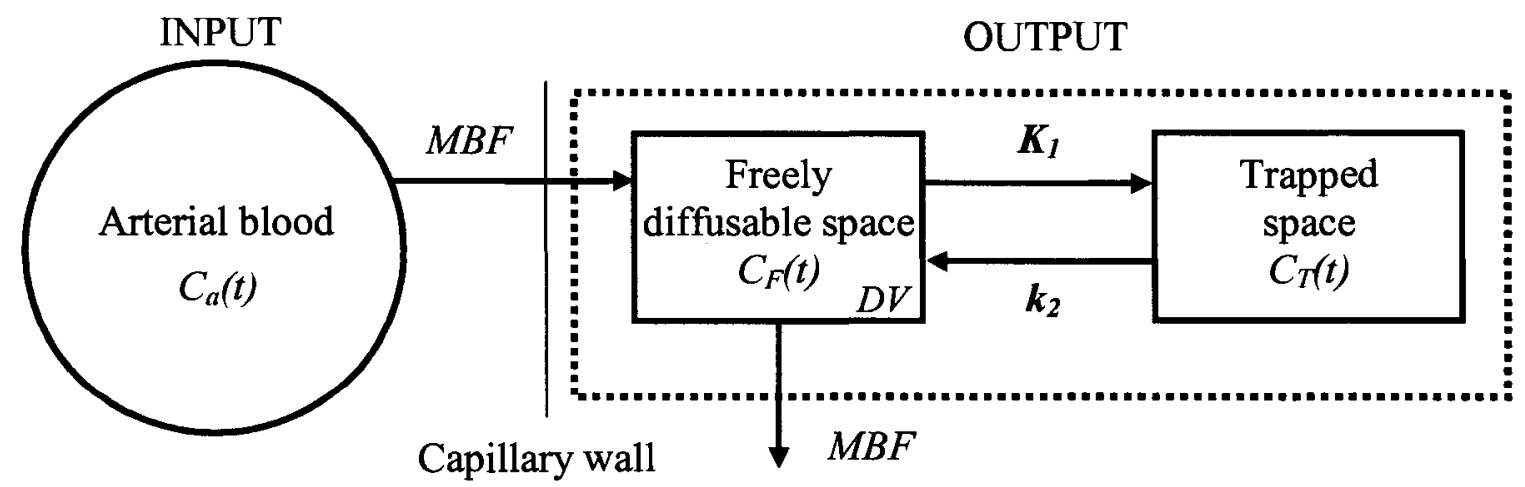

Figure 2.5: Alternate two-compartment model for ${ }^{13} \mathrm{NH}_{3} . K_{1}$ and $k_{2}$ reflect the transfer of tracer between the freely diffusible and trapped compartments [30]. 
The following equations characterize the rates of change within the model [30]:

$$
\begin{aligned}
& \frac{d C_{F}(t)}{d t}=-\frac{\left[\left(K_{1}+M B F\right) \times C_{F}(t)\right]}{D V}+k_{2} \times C_{T}(t)+M B F \times C_{a}(t) \\
& \frac{d C_{T}(t)}{d t}=\frac{\left[K_{1} \times C_{F}(t)\right]}{D V}-k_{2} \times C_{T}(t)
\end{aligned}
$$

The biexponential solution to this set of equations is expressed as [34]:

$$
C_{m}(t)=\frac{\left[M B F \times\left(K_{1}+k_{2}-A\right)\right]}{B-A} e^{-A \times t}+\frac{\left[M B F \times\left(B-K_{1}-k_{2}\right)\right]}{B-A} e^{-B \times t}
$$

where

$$
\begin{aligned}
& A, B=\frac{a+,-\sqrt{a^{2}-4 b}}{2}, \\
& a=\frac{M B F}{D V+K_{1}+k_{2}} \text { and } \\
& b=\frac{M B F}{D V \times k_{2}} .
\end{aligned}
$$

As in the previous two-compartment model, the measured concentration is expressed using the geometric model, which is combination of the arterial blood and myocardial concentrations, corrected for PV losses and spillover (Eq. 2.9). However, this model has added complexity since an additional parameter $(M B F)$ must be determined during the model fitting, in addition to the other parameters $\left(K_{l}, k_{2}, D V, F_{a}\right)$. Furthermore, in this model it is assumed that the reduction in ${ }^{13} \mathrm{NH}_{3}$ extraction at high flows is non-negligible due to slow metabolic conversion of the compound, thus $K_{I}$ must be corrected for tracer extraction in order to obtain a true estimate of MBF according to [26]: 
$K_{1}=M B F \times E(M B F)=M B F \times\left[1-0.607 e^{-1.25 / M B F}\right]$

Finally, rather than use the full blood and myocardial TACs, this model uses only the first 90 seconds, removing the need for a metabolite correction of the arterial input, $C_{a}(t)$.

Two-Compartment Model for Rubidium-82

A two-compartment model for ${ }^{82} \mathrm{Rb}$ that is equivalent to the alternate model for ${ }^{13} \mathrm{NH}_{3}$ has also been developed (Fig. 2.5) [33,34]. As in the alternate ${ }^{13} \mathrm{NH}_{3}$ model, $D V$ is the distribution volume in the first compartment and $C_{a}(t)$ is the arterial blood concentration. In contrast to the previous model, $C_{F}(t)$ and $C_{T}(t)$ are the tracer concentrations in the vascular and intracellular spaces, respectively. The blood flow is represented by $M B F$.

The solution to the model is given in Eq. 2.13, and the measured concentration follows Eq. 2.9. Use of this model has, however, been limited since large estimation errors arise when: 1) the model parameters are left free to be determined independently during the fitting, and 2) noise is present in the data, which in realistic terms is always the case. In light of these significant limitations, a simulation study was conducted in which it was determined that a simple one-compartment model was adequate for detecting differences in MBF [47].

\subsection{One-Compartment Models}

Another method that can be used to assess the kinetics of ${ }^{13} \mathrm{NH}_{3}$ is the one-compartment model (Fig. 2.6). This model is a simplification of the two-compartment model in that it 
disregards the metabolically bound space $\left(k_{3}=0\right)$ and it only uses the first four minutes of TAC data as input [29]. As in the initially presented two-compartment model, $K_{l}$ provides a direct estimate of $\mathrm{MBF}$ in $\mathrm{mL} / \mathrm{min} / \mathrm{g}$.

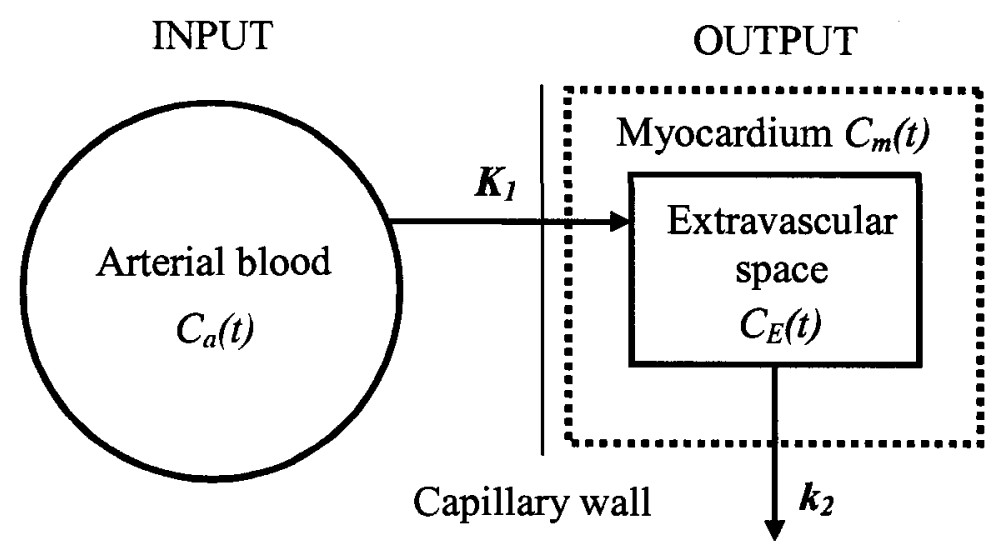

Figure 2.6: One-compartment model describing the uptake and retention of ${ }^{13} \mathrm{NH}_{3}$ in myocardial tissue. $K_{1}$ represents the exchange of ${ }^{13} \mathrm{NH}_{3}$ between the arterial blood and the extravascular space, and $k_{2}$ reflects the washout of ammonia from the extravascular space [29].

The single differential equation that describes this model is expressed as:

$$
\frac{d C_{m}(t)}{d t}=K_{1} C_{a}(t)-k_{2} C_{E}(t)
$$

The solution to this first-order differential equation is as follows [35]:

$$
C_{m}(t)=K_{1} e^{-k_{2} t} \otimes C_{a}(t)
$$

Similarly to the two-compartment model, the measured myocardial concentration follows Eq. 2.9, and a metabolite correction is applied to the arterial blood concentration.

As an alternate formulation of this model, the distribution volume parameter $D V$ (Eq. 2.10) can be used, whereby the myocardial concentration is: 


$$
C_{m}(t)=K_{1} e^{-\left(K_{1} / D V\right) t} \otimes C_{a}(t)
$$

With this method, only $K_{1}$ and $D V$ are required to be estimated during the model fitting. This method has been called the one-compartment DV model. As an additional measure to simplify model fitting, $D V$ can be set to a constant value, with the method then becoming the one-compartment constant-DV model, which requires solving for $K_{I}$ only.

Similarly to ${ }^{13} \mathrm{NH}_{3}$, MBF can be quantified with ${ }^{82} \mathrm{Rb}$ using the same one-compartment model depicted (Fig. 2.6) over the full time course of the scan (10 minutes) rather than the first 4 minutes only. To obtain accurate MBF estimates, $K_{I}$ is corrected for tracer extraction using the modified Renkin-Crone technique (Eq. 2.5). The following $P S$ function determines the unidirectional extraction of tracer from blood to tissue [35]:

$P S_{82} R b K_{1}(M B F)=0.63+0.26 M B F$

Additionally, as ${ }^{82} \mathrm{Rb}$ is not metabolized, a metabolite correction is not applied to the arterial blood concentration. This model was recently validated for the quantification of MBF in human subjects [35]. Finally, as with ${ }^{13} \mathrm{NH}_{3}$, the one-compartment DV and constant-DV models can also be used to describe the kinetics of ${ }^{82} \mathrm{Rb}$, with the exception that a metabolite correction is not required.

\subsection{Implementation of Compartment Models}

For the purposes of this research, the two-compartment model [28], one-compartment DV and one-compartment constant-DV models were used to generate estimates of MBF 
with ${ }^{13} \mathrm{NH}_{3}$. Similarly for ${ }^{82} \mathrm{Rb}$, the one-compartment, one-compartment $\mathrm{DV}$ and onecompartment constant-DV models were employed. These models were chosen as they were considered to be the most robust of all the variations. Additionally, the ${ }^{13} \mathrm{NH}_{3}$ twocompartment model was used as the gold-standard for validation of the MBF measurements obtained from the other ${ }^{13} \mathrm{NH}_{3}$ models and the ${ }^{82} \mathrm{Rb}$ method, as it characterizes the true physiology and is the current quantitative clinical imaging standard [48]. The selected models were implemented in MATLAB according to the modelspecific equations discussed above, and incorporated into the FlowQuantC software. The parameters of the models $\left({ }^{13} \mathrm{NH}_{3}: K_{l}, k_{2}\right.$ or $D V, k_{3}, F_{a} ;{ }^{82} \mathrm{Rb}: K_{1}, k_{2}$ or $\left.D V, F_{a}\right)$ were estimated iteratively using a non-linear weighted least squares technique [44] with weights equal to the square-root of the frame lengths.

The determination of solutions to the full compartment models often requires numerous iterations. This is a computationally intensive process that is not amenable for immediate interpretation and does not always result in convergence to a valid solution. It is for these reasons that the previously discussed simplified kinetic models were developed. However, these reduced kinetic models may still require a lengthy iterative process before reaching a final solution. This is undesirable for clinical use where the data of multiple patients must be processed daily. A more robust method is one that can be evaluated directly, in an analytical fashion, such as the simplified net retention model that is explored in the following chapter. 


\section{CHAPTER 3 - Development and Validation of a Simplified Net Retention Model}

\subsection{Principles of the Simplified Net Retention Model}

The net retention model is a simple first-pass method that is used to estimate the net retention rate (R) of a tracer in the myocardium by capturing the early tracer kinetics (Fig. 3.1). It is based on the assumption that all of the tracer is extracted from the blood into the tissue on the first transit of the tracer through the LV. This model is a simplification of the one-compartment method presented in the previous chapter; it assumes that washout, or back-diffusion, of tracer from the tissue does not occur, thus $k_{2}$ $=0$. Substituting this value into the solution of the one-compartment model (Eq. 2.16), and recognizing that the rate parameter linking the arterial blood pool in the myocardium is $R(\mathrm{~mL} / \mathrm{min} / \mathrm{g})$ rather than $K_{l}$, the following expression describing the myocardial concentration is obtained:

$$
C_{m}(t)=R \otimes C_{a}(t)
$$

Note that this expression is also evaluated at a particular time $T$, a further simplification from the compartment model. The parameter of interest, $R$, can then be computed directly, without the need for least-squares fitting, by rearranging Eq. 3.1 as follows [24]:

$$
R=\frac{C_{m}(T) / R C}{\int_{0}^{T} C_{a}(t) d t}=E \times M B F
$$


where $C_{a}(t)$ is the arterial blood concentration integrated from zero to time $T$. The parameter $R C$ has also been added to correct for partial volume losses. Using this relationship, the net retention values can then be corrected for extraction (E) as indicated to acquire an estimate of $\mathrm{MBF}$.

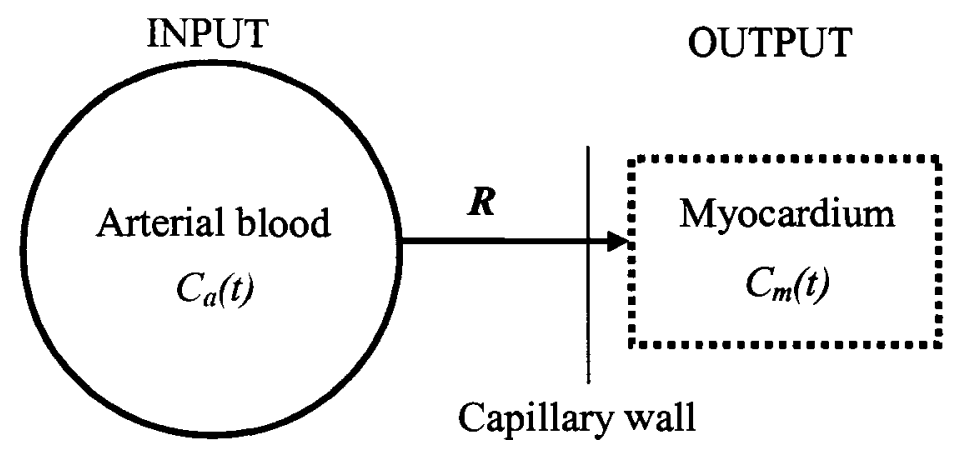

Figure 3.1: Simplified net retention model describing the uptake and retention of activity in myocardial tissue. $R$ represents the uptake of tracer from the arterial blood pool into the myocardial tissue.

This model is advantageous as it gives a physiologically relevant description of both the microvascular spaces within the tissue and the kinetic behaviour of extracted tracers, making it well suited for the analysis of MBF with ${ }^{82} \mathrm{Rb}$ and ${ }^{13} \mathrm{NH}_{3}$. Additionally, the method is simple and easily applicable in routine clinical use [24]. The development and implementation of this model for use with ${ }^{82} \mathrm{Rb}$ and ${ }^{13} \mathrm{NH}_{3}$ in this project are described in the proceeding sections. 


\subsection{Determination of a Correction for Partial Volume Losses}

To correct for myocardial partial volume loss, a single RC parameter was used. The RC value was determined using a cardiac motion simulation program. In brief, this program performed a convolution of the one-dimensional activity distributions (or profiles), defined with an arbitrary activity concentration of 1.0 , with the point spread function (PSF) of the scanner to generate a simulated PET image. This was accomplished by modeling the LV as an ellipsoid with $10 \mathrm{~mm}$ myocardial wall thickness at end-diastole, a sinusoidal LV volume curve and a Gaussian PSF with a $15.6 \mathrm{~mm}$ full width at half maximum (FWHM) [49]. Using this simulation, the RC value was determined to be 0.66 .

\subsection{Optimization of the Arterial Blood Integration Time}

The integration time, $T$, for the arterial blood concentration was determined by comparison with a modified gamma variate fit to the first-pass blood input curve. This method was first suggested by Thompson where he demonstrated that the transit time of the tracer in arterial blood following a bolus injection could be modeled using the probability density function representing a modified gamma variate relationship [50]:

$$
C_{a}(t)=\frac{A}{\beta^{\alpha} \times \Gamma(\alpha)} \times(t-\tau)^{(\alpha-1)} \times e^{-(t-\tau) / \beta}
$$

In this expression, $A$ represents the area under the arterial blood input curve up to an endpoint corresponding to $50 \%$ of the peak blood activity value, $\alpha$ and $\beta$ are distribution parameters that are determined during model fitting, $\tau$ is the delay time between the time 
of injection and the initial rapid rise in uptake of the tracer into the blood, and $\Gamma$ is the gamma function.

The gamma variate was used for the integration as it closely approximates the shape of the sharp peak of the blood input curve, which is typical of the case when the tracer is injected as a bolus; however, there are certain cases where the tracer infuses slowly rather than being a true bolus. Thus, a method that works reliably in both situations was required. A 'fixed-time past the peak' integration method was developed, as expressed in Eq. 3.1, where $T$ is set to a fixed time past the time of the peak activity of the arterial blood input curve. To validate this method, the net retention model was implemented in the FlowQuant $(\mathrm{C}$ software with both of the arterial blood integration techniques. The model was then applied to the rest and stress ${ }^{13} \mathrm{NH}_{3}$ data from 14 healthy volunteers first using the gamma variate integration of the blood input curve to determine the $R$ values for each subject. The gamma variate was fit to the blood input curves using a non-linear least squares algorithm, which estimated the distribution parameters $\alpha$ and $\beta$ and subsequently fit the model, expressed in Eq. 3.3, to the data to obtain the blood integral. An example of the gamma variate fit to the data of a normal subject is shown in Figure 3.2. The arterial blood curves which were best approximated by the gamma variate were then selected from the group of 14 subjects and the mean retention values across the group were tabulated. 


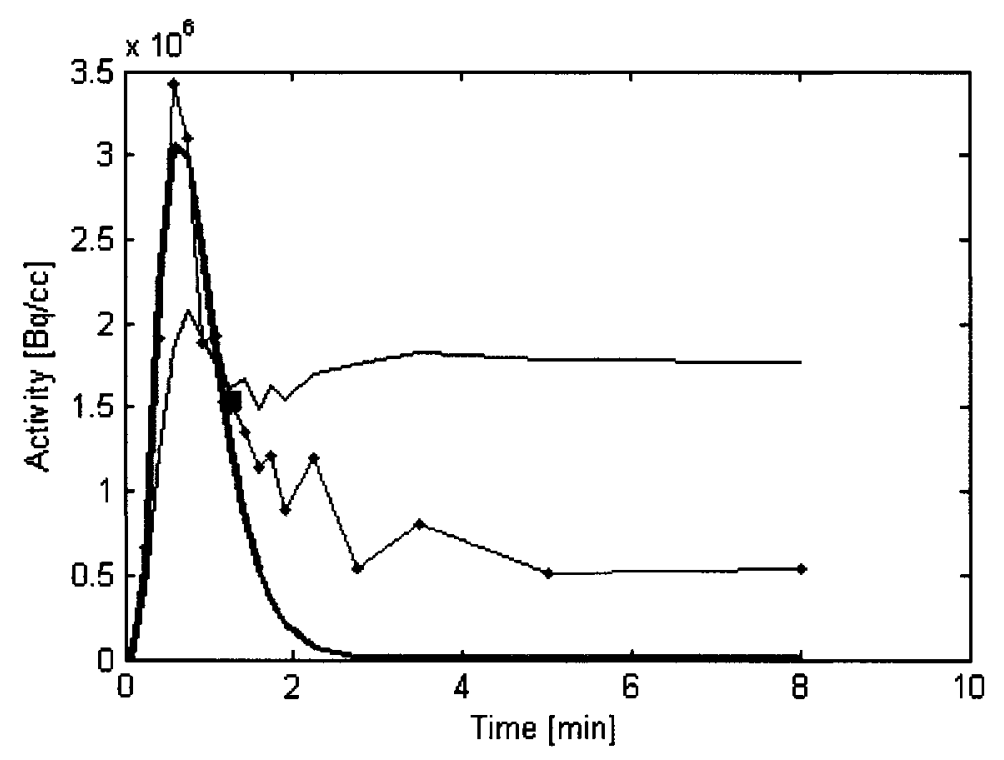

Figure 3.2: Gamma variate fit to the arterial blood input curve of a normal subject. The gamma variate fit is shown in green, with the endpoint indicated by a magenta block. The blood input curve is displayed in red, while the myocardial TAC is shown in blue.

The fixed-time integration method was subsequently applied to the selected group of subjects with an explicit integration of the arterial blood input curve, as shown in Eq. 3.2. The times tested were $1.1,1.15,1.2$ and 1.4 minutes past the arterial blood peak. These times were chosen based on the average of the gamma variate endpoint, as tabulated from the selected group of subjects. To determine the arterial blood integral value, integration was performed on the arterial blood TACs from the cavity, base and atrium of the LV.

The three integrals were then averaged to obtain a single value for $\int_{0}^{T} C_{a}(t) d t$ that was fully representative of the overall arterial blood activity in the interval of 0 to $T$, as displayed in Figure 3.3. The myocardial concentration was evaluated at the end integration time, $T$. The $R$ values obtained for the subjects using this technique were then averaged for each integration endpoint. 

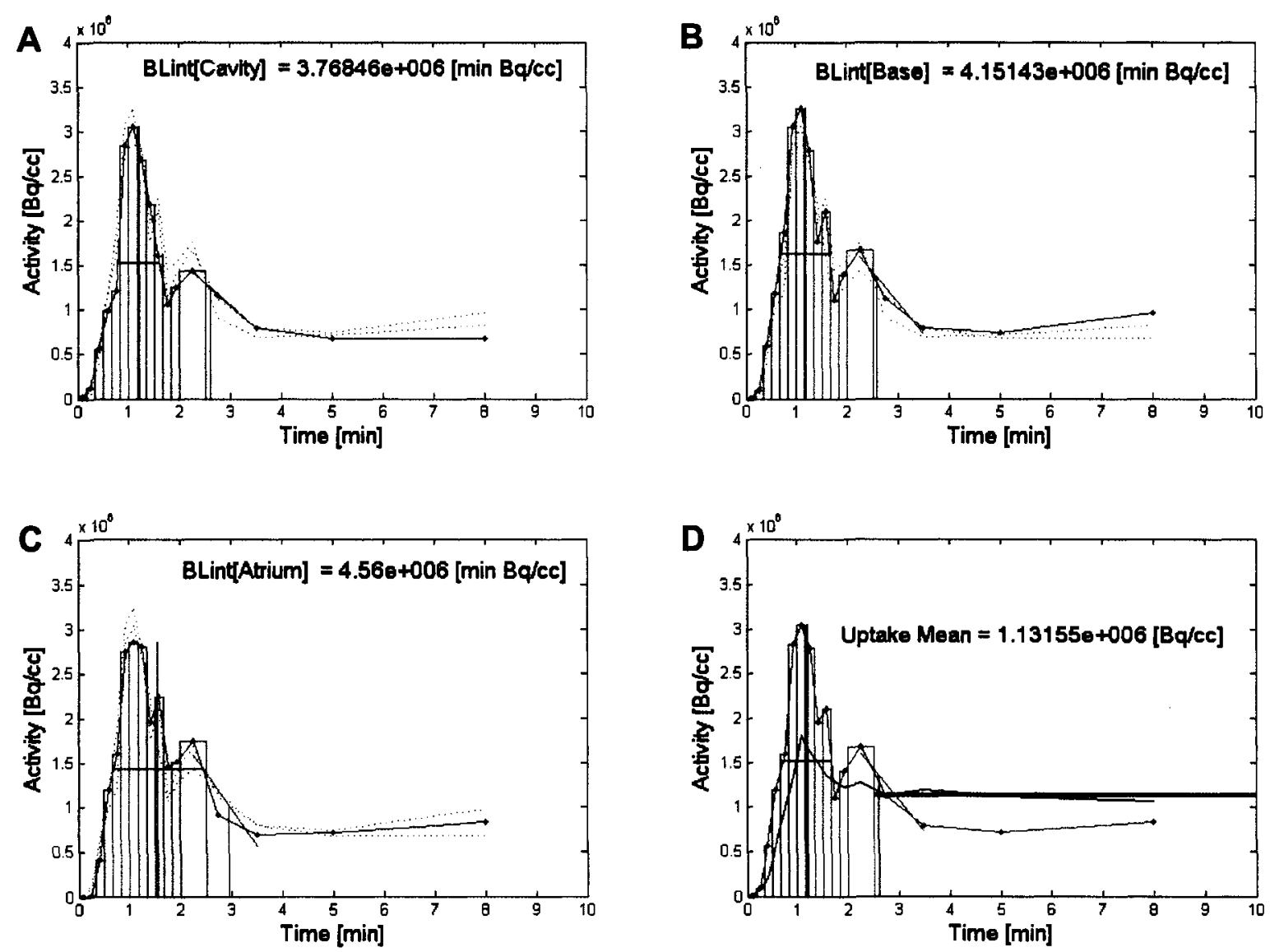

Figure 3.3: Net retention model output for ${ }^{82} \mathrm{Rb}$. Arterial blood TACs (solid red lines) are shown for the (A) cavity, (B) base, and (C) atrium regions of the $L V$. The dotted red lines in each regional plot indicate the shape of the blood TACs in the other regions. The period of integration from 0 to time $T$ is represented by the green lines. The value of the blood integral is given by BLint in units of min·Bq/cc. Averages of the curves from the three regions as well as the myocardial TAC (dark blue line) are depicted in (D). The cyan line denotes the time at which the myocardial TAC was evaluated.

Comparison of the mean retention values at rest and stress between the groups evaluated at different integration times (Table 3.1), indicated that a time of 1.1 minutes gave the most similar results to the gamma variate method. Therefore, for ${ }^{13} \mathrm{NH}_{3}$ data, the net retention model was applied with an integration endpoint of 1.1 minutes past the peak of 
the blood input curve. For ${ }^{82} \mathrm{Rb}$, the net extraction is lower and the blood pool clearance is slower than ${ }^{13} \mathrm{NH}_{3}$, therefore a longer blood integration time of $T=$ peaktime +1.4 minutes was determined using similar methods.

Table 3.1: Mean retention values derived from retention for the gamma variate and the fixed-time past the peak integration methods for ${ }^{13} \mathrm{NH}_{3}$

\begin{tabular}{c|c|c|c|c|c}
\hline \multirow{2}{*}{ Retention } & Gamma & \multicolumn{4}{|c}{ Integration times past the peak (min.) } \\
& variate & 1.4 & 1.2 & 1.15 & 1.1 \\
\hline Rest & $\mathbf{0 . 6 6}$ & 0.59 & 0.62 & 0.63 & $\mathbf{0 . 6 4}$ \\
Stress & $\mathbf{1 . 5 7}$ & 1.32 & 1.39 & 1.41 & $\mathbf{1 . 4 3}$ \\
\hline
\end{tabular}

\subsection{Development of Net Extraction Corrections}

To obtain an accurate flow estimate with the retention model, $R$ must be corrected for less than $100 \%$ extraction of the tracer from blood into the myocardium; this typically occurs at high flow values as discussed in Chapter 2, section 2.2. Thus, the extraction correction is a function of flow and it is expressed using a modified Renkin-Crone model (Eq. 2.5). This function is also used as a method of standardizing the $R$ estimates to the flow values from the compartmental models.

In order to determine the $P S$ products that characterize the tracer-specific extraction functions for ${ }^{13} \mathrm{NH}_{3}$ and ${ }^{82} \mathrm{Rb}$, estimates of the constants $A$ and $B$ were required. To perform this estimation, Eq. 2.5 was fit to the median rest and stress values of $R$ in 14 healthy volunteers versus the corresponding MBF data from the one-compartment model for ${ }^{82} \mathrm{Rb}$, and versus the MBF results from the two-compartment model for ${ }^{13} \mathrm{NH}_{3}$. However, the curves gave extraction values that were greater than 1 at low flows, which 
is physiologically impossible. The reason for this was postulated to be an underestimation of the arterial blood integral due to recirculation and subsequent uptake of tracer after the first-pass through the heart. To correct for this underestimation, the derived extraction curve for ${ }^{13} \mathrm{NH}_{3}$ was compared to the accepted curve of Schelbert et al. [26] that was determined in dogs. At a typical resting flow value of $0.7 \mathrm{~mL} / \mathrm{min} / \mathrm{g}$, the published curve gave an extraction fraction of 0.9 . The ratio of the two extraction curves at this value was then taken and was determined to be 1.15 . Thus, to obtain the same results with the retention values derived in this study, it was determined that a multiplicative factor of 1.15 was required to correct the underestimated arterial blood integrals, as follows:

$$
R=\frac{C_{m}(T) /(R C \times 1.15)}{\int_{0}^{T} C_{a}(t) d t}
$$

To retain consistent methodology between the two tracers, the factor of 1.15 was also used when applying the net retention model to ${ }^{82} \mathrm{Rb}$ data. Once this factor was determined, the retention method, as expressed in Eq 3.4, was reapplied to the healthy subject data to generate revised estimates of $R$. Then, by fitting the extraction model to this data, the corrected $P S$ functions characterizing the tracer-specific net extraction for the retention model were then found to be:

$$
P S_{8_{2} R B R}(M B F)=0.74+0.083 M B F
$$

and

$$
P S_{{ }^{13}{ }_{N H_{3} R}}(M B F)=1.55+0.11 M B F
$$


The net extraction functions and net retention curves are shown in Figure 3.4. The net extraction curves (Fig. 3.4 A) demonstrate a good fit to the median rest and stress $R$ versus MBF data for both ${ }^{82} \mathrm{Rb}\left(\mathrm{r}^{2}=0.96\right)$ and ${ }^{13} \mathrm{NH}_{3}\left(\mathrm{r}^{2}=0.87\right)$. Similarly accurate fits are shown for the net retention $(E \times M B F)$ curves (Fig. 3.4 B) for the two tracers $\left({ }^{82} \mathrm{Rb} \mathrm{r}{ }^{2}\right.$ $=0.87$ and ${ }^{13} \mathrm{NH}_{3} \mathrm{r}^{2}=0.86$ ), indicating that the regression line is representative of the data. From this figure it is evident that ${ }^{13} \mathrm{NH}_{3}$ is more highly retained than ${ }^{82} \mathrm{Rb}$ as flow increases, as expected.

The extraction fractions corresponding to these derived $P S$ products were used to determine MBF values for each subject from the $R$ estimates with the aid of a lookup table. A lookup table uses an array of data to map input values to output values. Given input values, the function performs a 'lookup' operation to retrieve the corresponding output values from the table. If the lookup table does not define the input values, the output values are estimated based on interpolation of neighbouring table values [51]. In this case the table is formed by evaluating the extraction correction function at various MBF values, from 0 to $5 \mathrm{~mL} / \mathrm{min} / \mathrm{g}$, to obtain net retention rate values. Then, by inputting an experimentally determined net retention rate, the corresponding MBF value is determined by looking up the $R$ value in the table. 

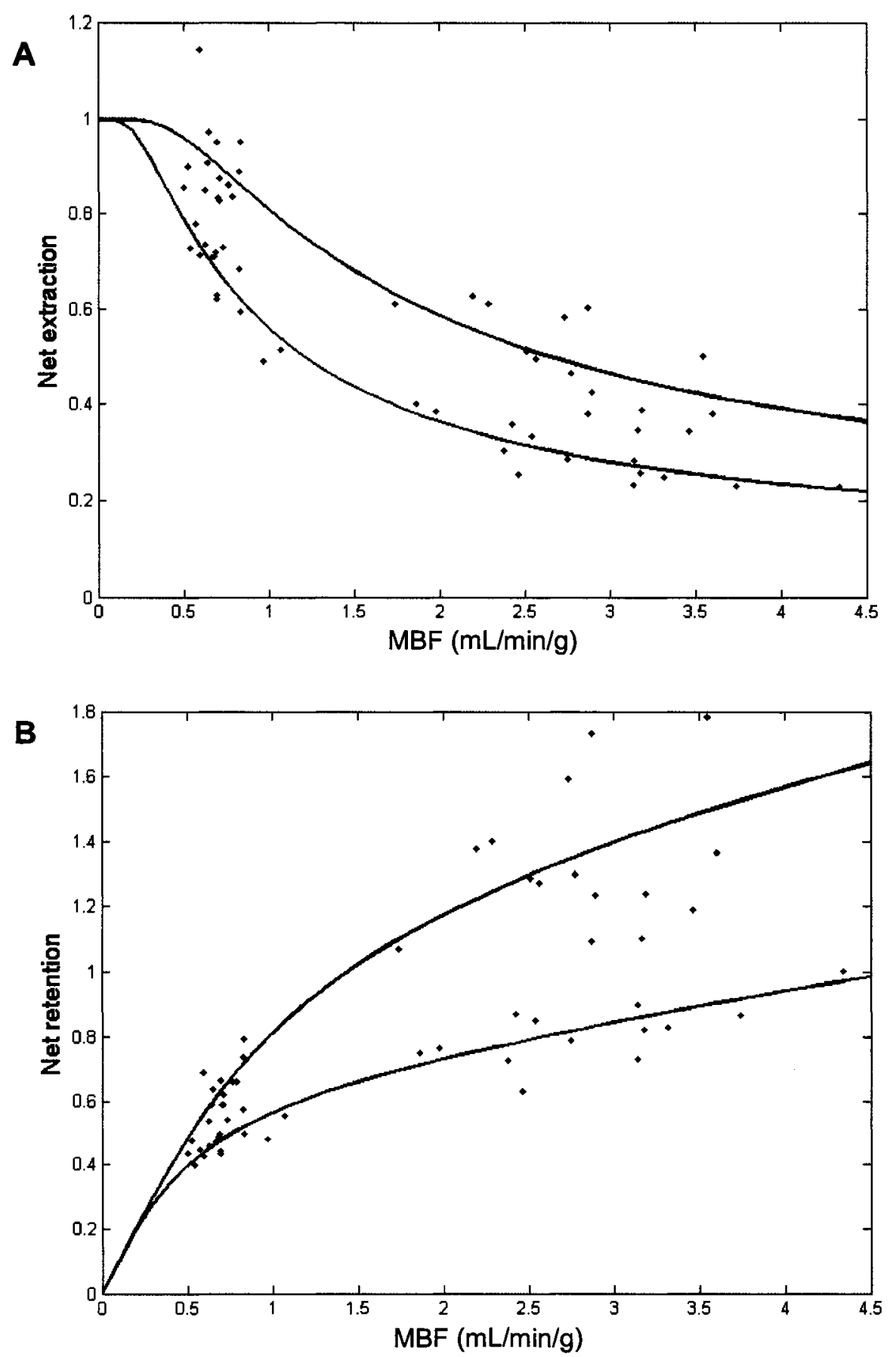

Figure 3.4: (A) Derived extraction curves for ${ }^{13} \mathrm{NH}_{3}$ retention (R) model (dark blue) as compared to the ${ }^{82} \mathrm{Rb}$ retention ( $R$ ) model (teal). (B) Net retention ( $E \times M B F$ ) curves for the ${ }^{13} \mathrm{NH}_{3}$ retention $(R)$ model and the ${ }^{82} \mathrm{Rb}$ retention $(R)$ model. The dots represent the mean individual subject values for the 14 healthy volunteers. 
Figure 3.5 shows the difference between ${ }^{82} \mathrm{Rb}$ and ${ }^{13} \mathrm{NH}_{3}$ extraction in the myocardial time-activity curves at stress for an individual subject. At a flow value of $2.2 \mathrm{~mL} / \mathrm{min} / \mathrm{g}$ (mean of MBF derived from retention for ${ }^{82} \mathrm{Rb}$ and ${ }^{13} \mathrm{NH}_{3}$ in that subject), the ratio of the tracer activities late after injection (relative retention) $(65 \%)$ is similar to the ratio of the net retention fractions (Fig. 3.4 B) (72\%), further demonstrating the concurrence of our derived extraction functions with the measured data.

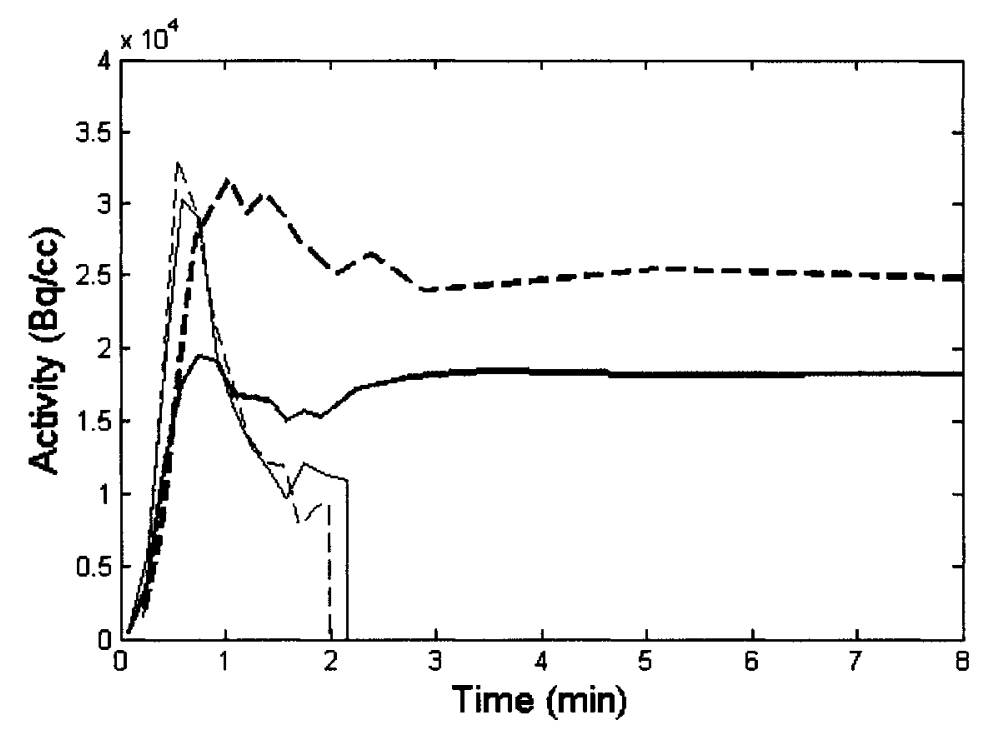

Figure 3.5: Comparison of ${ }^{13} \mathrm{NH}_{3}$ and ${ }^{82} \mathrm{Rb}$ net retention at stress for an individual subject showing that the ratio of the myocardial TACs for ${ }^{13} \mathrm{NH}_{3}$ (dark blue dashed line) and ${ }^{82} \mathbf{R b}$ (teal dashed line) is similar to the ratio between the net retention functions for the two tracers. Myocardial time-activity curves were normalized to the area under the respective blood time-activity curves as shown $\left({ }^{13} \mathrm{NH}_{3}\right.$ - dashed red line; ${ }^{82} \mathbf{R b}$ - solid red line), to remove any variability due to differences in injection rates. 


\subsection{Validation of Net Extraction Corrections}

To determine the applicability of the derived extraction corrections for ${ }^{13} \mathrm{NH}_{3}$ and ${ }^{82} \mathrm{Rb}$ in all cases, not just in normal subjects, the curves required subsequent validation. The net extraction curves were verified in a group of $\mathrm{CAD}$ patients consisting of 13 male subjects, $39-78$ years old, with evidence of CAD documented by coronary angiography or relative myocardial perfusion imaging. Subjects underwent both rest and stress ${ }^{82} \mathrm{Rb}$ and ${ }^{13} \mathrm{NH}_{3}$ PET imaging. Images were acquired and processed as detailed in Chapter 1. The retention model was then applied to the processed data to generate estimates of $R$ for each patient. To obtain estimates of $\mathrm{MBF}$, the previously described ${ }^{82} \mathrm{Rb}$ onecompartment model and the ${ }^{13} \mathrm{NH}_{3}$ two-compartment model were used. To account for the inhomogeneous measurements in the $\mathrm{CAD}$ patients due to the presence of disease in certain regions of the $\mathrm{LV}$, the retention rate and MBF polar maps were divided into 17 segments, as described in Appendix A, and average $R$ and MBF values were computed for each segment.

The segmental $R$ estimates were plotted versus the segmental MBF estimates for each tracer and the extraction model was fit to the two datasets according to the methods discussed in the previous section for the normal population extraction curves. The resultant fitted functions are shown in Figures 3.6 (A) and (B) for ${ }^{82} \mathrm{Rb}$ and ${ }^{13} \mathrm{NH}_{3}$, respectively. Also displayed are the extraction curves derived in the normal subjects, as well as their corresponding $95 \%$ confidence bounds and $95 \%$ non-simultaneous prediction bounds. 
A

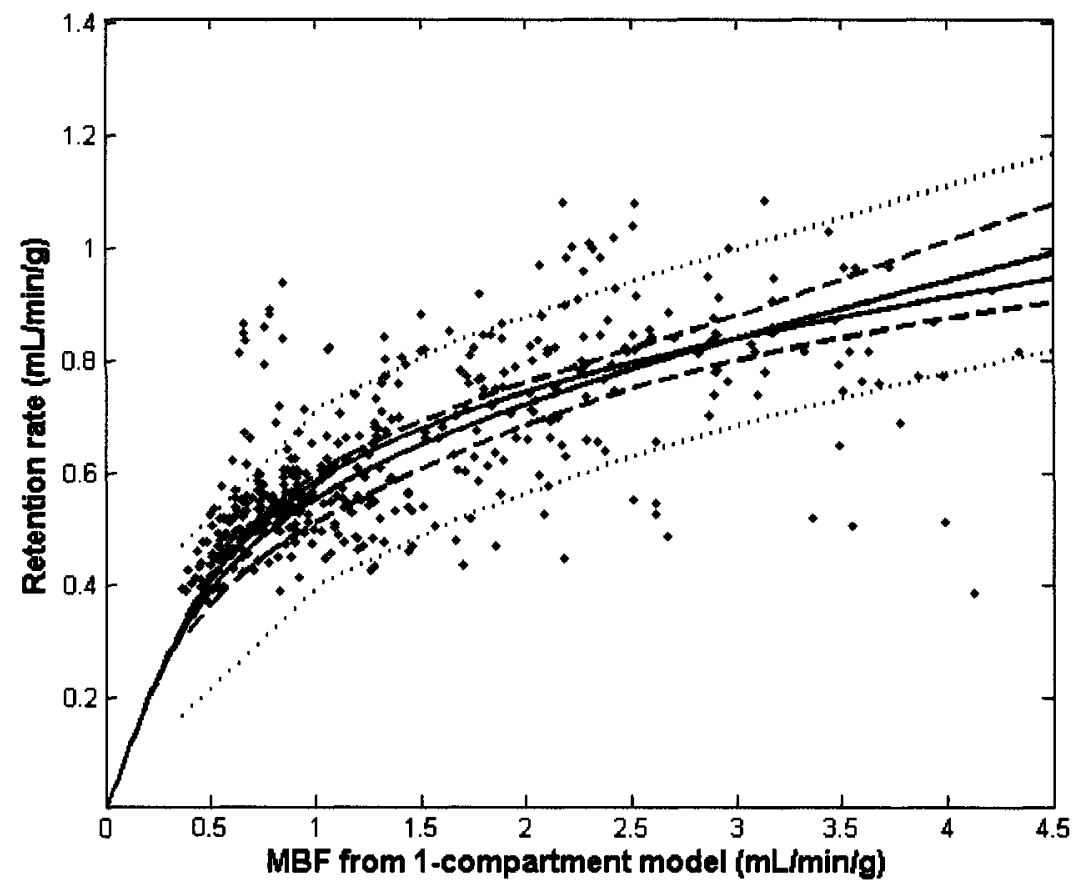

$\mathbf{B}$

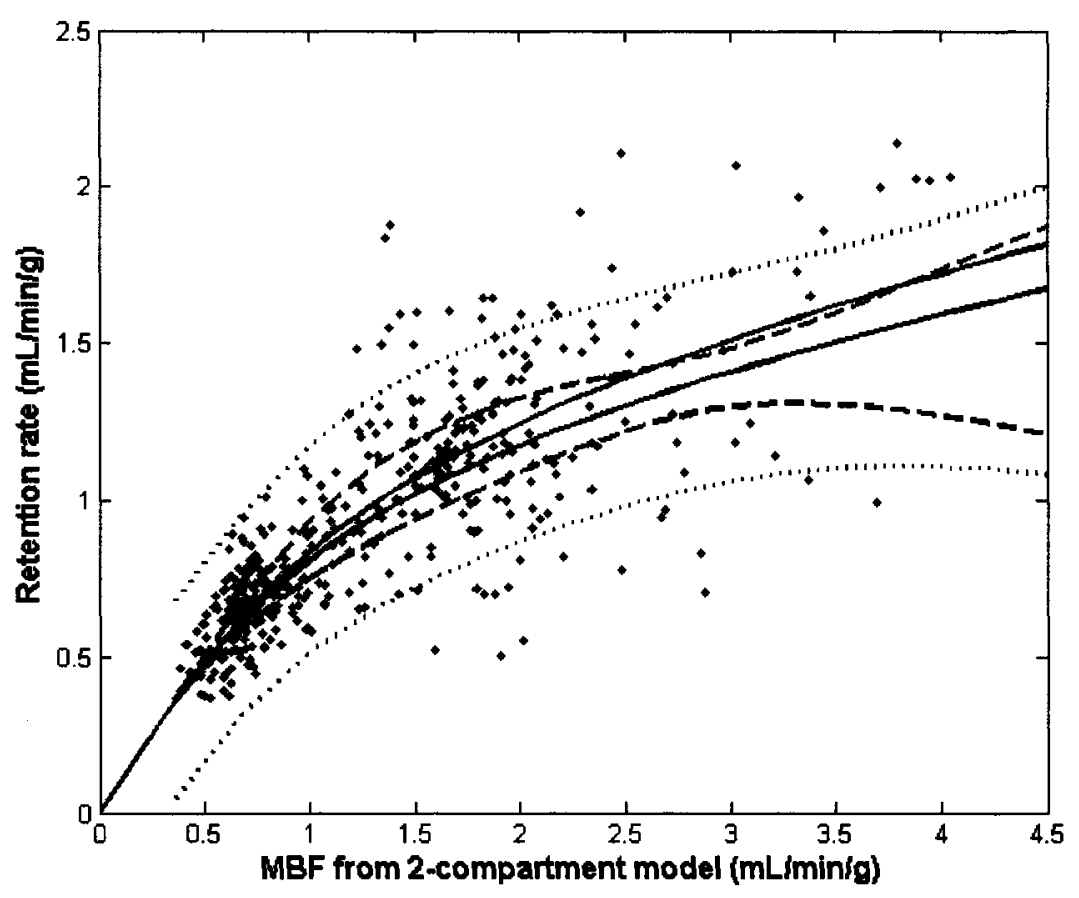

Figure 3.6: Extraction curves for CAD patients compared with the curves from healthy volunteers for (A) ${ }^{82} \mathrm{Rb}$ and (B) ${ }^{13} \mathrm{NH}_{3}$. CAD segmental $R$ values (red points) are plotted vs. MBF values obtained with the one- and two-compartmental models, respectively. The solid green line is the best fit to the CAD data. Solid black lines show the healthy subject curves; dashed black lines are $95 \%$ confidence bounds and dotted black lines are non-simultaneous prediction bounds for these functions. 
As demonstrated for ${ }^{82} \mathrm{Rb}$, the extraction curve that was independently fit to the $\mathrm{CAD}$ data falls entirely within the confidence bounds on the function estimated from the healthy volunteer data, and the majority of the individual subject values fall within the non-simultaneous predictions bounds. For ${ }^{13} \mathrm{NH}_{3}$, the CAD extraction curve strays slightly outside the confidence bounds for the healthy volunteer curve at high flows. This is likely explained by the presence of some outliers at high flows caused by convergence difficulties encountered by the optimization algorithm in certain segments when attempting to fit the ${ }^{13} \mathrm{NH}_{3}$ two-compartment model to the $\mathrm{CAD}$ data. Nonetheless, these results indicate that the extraction curves derived from the healthy subjects can be used to describe the relationship between the retention rate and compartmental MBF values obtained from the CAD subjects. Consequently, this validates the use of the extraction curves obtained in normal subjects to estimate MBF values in all subjects using the retention model.

\subsection{Discussion}

A modified methodology for the net retention model has been developed. Accordingly, tracer-specific extraction corrections for ${ }^{82} \mathrm{Rb}$ and ${ }^{13} \mathrm{NH}_{3}$ were derived using this model in healthy subjects, and were subsequently validated in CAD patients. A limitation of this method is that the net extraction functions are specific to the derived values of RC and $T$. However, the same methodology was used for both tracers; peaktime accounts for variability in the scan start time, and $T$ accounts for differences in blood clearance rates 
between the tracers. The $\mathrm{RC}$ value was comparable to the median PV correction $\left(1-F_{a}\right)$ value of 0.60 obtained from the ${ }^{13} \mathrm{NH}_{3}$ and ${ }^{82} \mathrm{Rb}$ compartment models.

As compared with previous studies, the net extraction curve derived in this project for the net retention model for ${ }^{82} \mathrm{Rb}$ is slightly higher than the previously published curve (Fig. 3.7) of Yoshida [24], implying that ${ }^{82} \mathrm{Rb}$ may be more highly extracted and thus may have better uptake imaging properties than previously shown, particularly at rest. The function derived by Yoshida was determined using a similar retention model to the one presented in this study, however it included a myocardium-to-blood spillover factor not contained in the present work. Additionally, our integration endpoint of $T=$ peaktime +1.4 minutes differs from the 1 minute integration time used by Yoshida [24], demonstrating dependence of the model on the chosen integration time. It is also significant to note that the function derived by Yoshida was based on TACs collected from external probes in open-chest dogs, whereas the function determined in this case was derived from human PET data. These differences in methodology may account for the discrepancy between the net extraction curve of Yoshida and the one presented in this work.

Conversely, the results obtained in the present study for ${ }^{13} \mathrm{NH}_{3}$ using the same retention model methodology in the same subjects suggest that net extraction of this tracer may be slightly lower than previously published [26,45,52-54]. The net extraction functions previously derived by Schelbert [26] and Gewirtz [45] were determined using a variation of the two-compartment model utilized in this study, where $K_{I}$ is an estimate of the net retention rate rather than flow. Also, their functions were derived using canine and swine 
data, respectively. The "exponential" integration approach used by Bellina [52], where a gamma variate was fit to the in-vivo PET data and an exponential was used to describe the downslope of in-vitro data, differs from the explicit integration over the blood input curve used in this study, indicating that the measured extraction function is also dependent on the type of integration used. Additionally, the integration endpoint of $T=$ peaktime +1.1 minutes used in this study differs from that used by Nienaber [53] of 1 minute, further demonstrating dependence of the model on the chosen integration time. Regardless of this limitation, the net retention model has been shown to generate precise flow estimates [24]. The utility of this model for the formation of a normal population database is discussed in the subsequent chapter. 


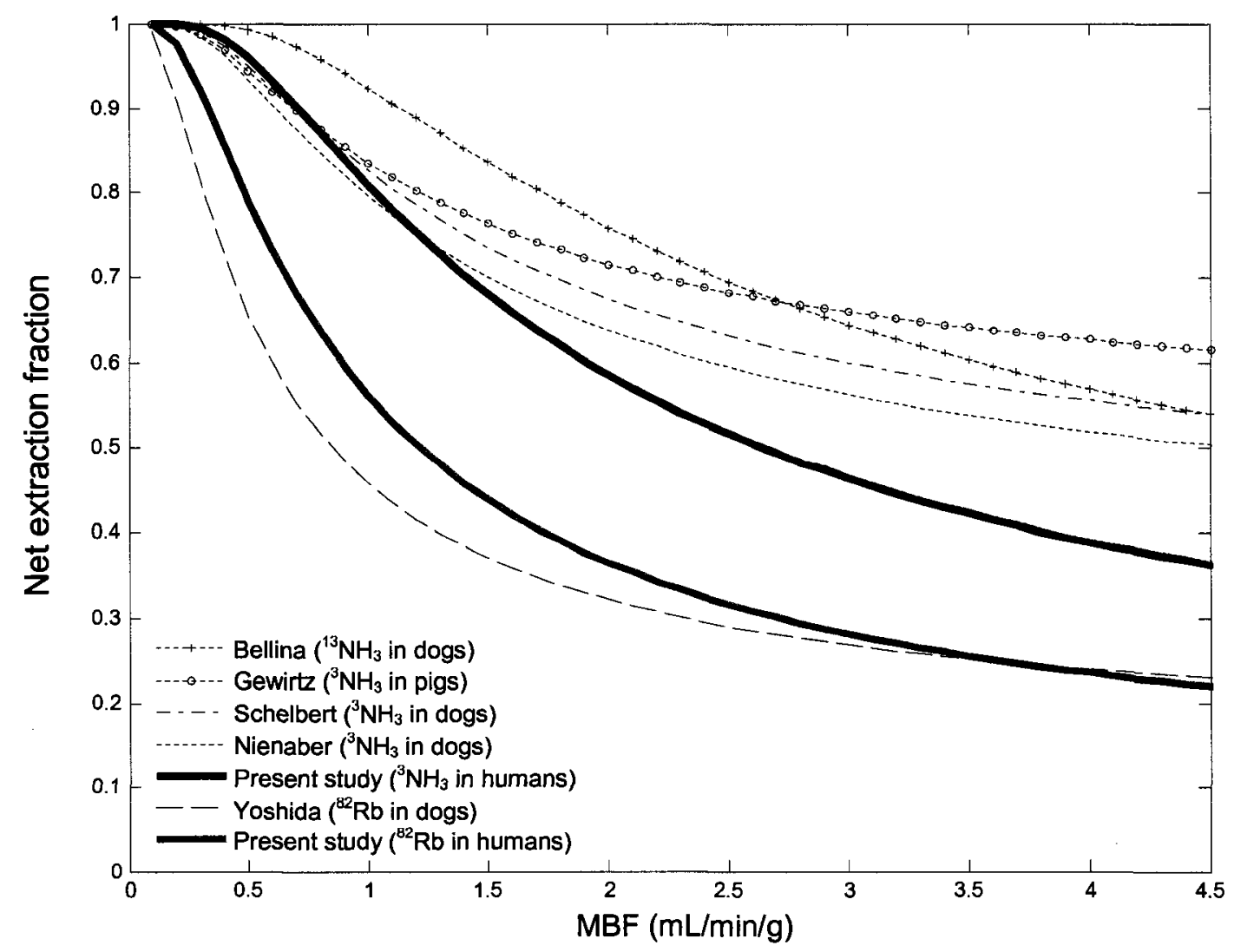

Figure 3.7: Comparison of extraction curves from previous studies in animals and the present study in humans. 


\section{CHAPTER 4 - Criteria and Construction of Normal Population Databases}

\subsection{Purpose and Utility of Normal Population Databases}

Computer-aided interpretation programs are commonly used in nuclear cardiology to aid in the diagnosis of $\mathrm{CAD}$, particularly with relative perfusion single-photon emission computed tomography (SPECT) imaging. Patient perfusion data is compared with a normal population database, which is a polar-map defining the regional lower limit of normal blood flow. From this comparison, regions of abnormality in the LV can be detected and localized. However, diagnosis of coronary disease and microvascular dysfunction may be improved by comparing myocardial perfusion scans with a database defining the lower limit of absolute normal MBF and MFR. To date, such diagnostic databases have only been reported using ${ }^{13} \mathrm{NH}_{3}$ PET and compartmental modeling [21]. In practice, the measured normal range of flow is determined both by the true physiological variability as well as the uncertainty in estimating the model parameters.

To determine the normal flow range, the mean and standard deviation of flow values from healthy subjects are required. Normal limits are then typically defined as the regions representing two standard deviations (SD) below the population mean. In patients, sectors of the myocardium that fall below this normal range are considered to be abnormal and thus representative of disease. 
The purpose of this research was to determine if the use of the simplified net retention model would reduce the apparent range of normal flow as compared to the compartmental models, potentially enhancing the ability to identify regional flow abnormalities in patients. The next sections detail the development of these databases and the results of their comparisons.

\subsection{Database Development}

Fourteen healthy volunteers ( 7 men, 7 women; $23-44$ years old; mean \pm SD age $=31 \pm$ 6 years) were enrolled to acquire data for database formation. The likelihood of CAD was less than $5 \%$ in all cases [55] and all resting electrocardiograms were normal. Each subject underwent rest and stress imaging with both ${ }^{13} \mathrm{NH}_{3}[56]$ and ${ }^{82} \mathrm{Rb}$ [3]. Scans were performed in a randomized order within a 2 -week period (median period $=2.5$ days). Subjects were instructed to fast for a minimum of 6 hours and to abstain from products containing caffeine for no less than 12 hours prior to imaging, as caffeine is the antidote to the pharmacologic stress agent. Maximal stress flow was induced with dipyridamole $(0.14 \mathrm{mg} / \mathrm{kg} / \mathrm{min} \times 5 \mathrm{~min})$. Stress scans were started 3 minutes after dipyridamole infusion; the antidote, aminophylline, was administered 4 minutes afterward to reverse the vasodilation.

${ }^{13} \mathrm{NH}_{3}$ and ${ }^{82} \mathrm{Rb}$ had similar infusion profiles, with an infusion time of $30 \mathrm{~s}$ and a total delivered activity of $220 \mathrm{MBq} .{ }^{13} \mathrm{NH}_{3}$ was injected with a syringe pump while ${ }^{82} \mathrm{Rb}$ was administered with a custom infusion system that delivered the tracer at a constant activity 
rate $[17,57]$. The activity and infusion rates were chosen to limit detector dead-time to less than $50 \%$ so that the peak first-pass blood activity could be measured accurately in the LV cavity for later quantification purposes.

The dynamic rest and stress PET data were acquired in $3 \mathrm{D}$ mode according to the protocols for ${ }^{82} \mathrm{Rb}$ and ${ }^{13} \mathrm{NH}_{3}$ discussed in Chapter 1, section 1.3. A 4-minute ${ }^{137} \mathrm{Cs}$ transmission scan was performed prior to the rest scan to correct for attenuation during the rest study. For imaging with ${ }^{82} \mathrm{Rb}$, a second 4-minute transmission scan was performed immediately following the stress scan to correct for attenuation during the stress study. For ${ }^{13} \mathrm{NH}_{3}$ imaging, the second transmission scan (4 minutes transmission, 4 minutes emission contamination) was performed prior to dipyridamole injection. An interval of 50 minutes between ${ }^{13} \mathrm{NH}_{3}$ infusions allowed for isotope decay. Images were corrected, reconstructed and processed using the FlowQuant $\mathbb{C}$ software as discussed in Chapter 1, sections 1.4 and 1.5 .

For scans performed with ${ }^{82} \mathrm{Rb}$ and ${ }^{13} \mathrm{NH}_{3}$, MBF was quantified using the retention model, one-compartment DV, and one-compartment constant-DV models. Additionally, for ${ }^{82} \mathrm{Rb}$ the one-compartment model was used, and for ${ }^{13} \mathrm{NH}_{3}$ the two-compartment model was used as the gold standard method. Polar maps representing rest, peak dipyridamole stress, and myocardial reserve (stress/rest) for $R, K_{1}$ and MBF, as well as their associated SDs, were generated for all tracer and model combinations as appropriate, forming normal population databases. From the polar maps, the median global values were calculated for each subject. Global (LV-median) and regional (polar 
map) population mean $\pm \mathrm{SD} \%$ values were used to evaluate the lower limit of normal flow (mean - 2SD). Global values were determined using the LV-median of the rest flow, stress flow, and MFR polar maps for each subject. For each model, the means and SDs were then calculated across subjects to obtain the global results. The regional values were determined by first computing the mean and standard deviation polar maps of the rest flow, stress flow, and MFR estimates across all subjects. The average values of the mean and standard deviation polar maps were then computed to obtain the regional results. The values are reported as normal population mean and SD.

Normal ranges and lower limits of normal were compared between the tracer and model combinations to assess the ability of each database to detect and localize disease. Mean retention rate and flow values from the compartment models were compared using the $t$ test. The variability associated with the databases was compared using the $F$-test. The variability is presented as $\mathrm{SD} /$ mean to adjust for the differences in the means between the parameters for the individual databases. A difference was considered statistically significant if the $p$ value was less than 0.05 . Additionally, the regional uniformity of the databases was assessed using the coefficient of variation (COV).

\subsection{Results}

The global LV-median results for the ${ }^{82} \mathrm{Rb}$ and ${ }^{13} \mathrm{NH}_{3}$ databases are provided in Table 4.1. Comparison of the MBF and MFR data between the MBF models indicates that the mean values are generally similar, as expected. For the compartment models, the regional 
normal ranges (SD) are shown in Table 4.2 and all are increased in comparison with the global values (Table 4.1$)$. This is due to the increase in variability $(+1$ to $+14 \%$ SD) in the estimates from added regional noise compared to the global LV-median values. This suggests that disease localization (with the regional database) may be less sensitive than disease detection (with the global database) alone. In contrast to the compartment model analysis, the regional variability for the net retention rates (Table 4.2) remained essentially unchanged as compared with the global values (Table 4.1), suggesting that the net retention values are more uniform over the entire LV than the results obtained with the compartment models.

Table 4.1: Global (LV-median) normal ranges and lower limits of normal for the retention and compartment models

\begin{tabular}{|c|c|c|c|c|c|c|}
\hline \multirow[t]{2}{*}{ Parameter } & \multicolumn{2}{|c|}{ REST } & \multicolumn{2}{|c|}{ STRESS } & \multicolumn{2}{|c|}{ STRESS / REST } \\
\hline & $\begin{array}{l}\text { mean } \pm \\
\text { SD (\%) }\end{array}$ & $\begin{array}{l}\text { mean } \\
-2 \text { sd }\end{array}$ & $\begin{array}{l}\text { mean } \pm \\
\text { SD (\%) }\end{array}$ & $\begin{array}{l}\text { mean } \\
-2 \text { sd }\end{array}$ & $\begin{array}{l}\text { mean } \pm \\
\text { SD }(\%)\end{array}$ & $\begin{array}{l}\text { mean } \\
-2 \text { sd } \\
\end{array}$ \\
\hline${ }^{82} R b R$ & $0.47 \pm 9$ & 0.38 & $0.83 \pm 14$ & 0.60 & $1.78 \pm 15$ & 1.25 \\
\hline${ }^{82} R b K_{1}$ & $0.48 \pm 12$ & 0.36 & $1.10 \pm 15$ & 0.77 & $2.32 \pm 21$ & 1.33 \\
\hline${ }^{82} R b K_{1}-D V$ & $0.49 \pm 20$ & 0.29 & $1.05 \pm 10$ & 0.83 & $2.19 \pm 17$ & 1.47 \\
\hline${ }^{82} R b K_{1}-D V$ const. & $0.48 \pm 20$ & 0.29 & $1.03 \pm 11$ & 0.80 & $2.17 \pm 18$ & $\mathbf{1 . 4 0}$ \\
\hline${ }^{13} \mathrm{NH}_{3} \mathrm{R}$ & $0.63 \pm 13$ & 0.46 & $1.35 \pm 16$ & 0.92 & $2.16 \pm 15$ & 1.50 \\
\hline${ }^{82} \mathrm{Rb} M B F_{R}$ & $0.71 \pm 20$ & 0.43 & $3.01 \pm 38$ & 0.72 & $4.26 \pm 34$ & 1.32 \\
\hline${ }^{82} R b M B F_{K 1}$ & $0.71 \pm 22$ & 0.40 & $2.88 \pm 23$ & 1.56 & $4.21 \pm 33$ & 1.45 \\
\hline${ }^{82} R b M B F_{K I}-D V$ & $0.73 \pm 37$ & 0.20 & $2.67 \pm 16$ & 1.80 & $3.86 \pm 26$ & 1.82 \\
\hline${ }^{82} R b M B F_{K 1}-D V$ const. & $\mathbf{0 . 7 3} \pm \mathbf{3 5}$ & 0.22 & $2.60 \pm 18$ & 1.68 & $3.78 \pm 30$ & 1.54 \\
\hline${ }^{13} \mathrm{NH}_{3} \mathrm{MBF}_{\mathrm{R}}$ & $0.70 \pm 17$ & 0.46 & $2.96 \pm 42$ & 0.49 & $4.14 \pm 40$ & 0.84 \\
\hline${ }^{13} \mathrm{NH}_{3} \mathrm{MBF}-\mathrm{DV}$ & $0.65 \pm 18$ & 0.41 & $2.26 \pm 16$ & 1.54 & $3.61 \pm 23$ & 1.95 \\
\hline${ }^{13} \mathrm{NH}_{3} \mathrm{MBF}-\mathrm{DV}$ const. & $0.61 \pm 15$ & 0.42 & $2.28 \pm 16$ & 1.53 & $3.78 \pm 21$ & 2.17 \\
\hline${ }^{13} \mathrm{NH}_{3} \mathrm{MBF}$ & $0.71 \pm 13$ & 0.52 & $2.79 \pm 19$ & 1.74 & $3.97 \pm 21$ & 2.27 \\
\hline
\end{tabular}


Table 4.2: Regional (polar map) normal ranges and lower limits of normal for the retention and compartment models

\begin{tabular}{|c|c|c|c|c|c|c|}
\hline \multirow[t]{2}{*}{ Parameter } & \multicolumn{2}{|c|}{ REST } & \multicolumn{2}{|c|}{ STRESS } & \multicolumn{2}{|c|}{ STRESS / REST } \\
\hline & $\begin{array}{l}\text { mean } \pm \\
\text { SD (\%) }\end{array}$ & $\begin{array}{l}\text { mean } \\
-2 \text { sd }\end{array}$ & $\begin{array}{l}\text { mean } \pm \\
\text { SD (\%) }\end{array}$ & $\begin{array}{l}\text { mean } \\
-2 \text { sd }\end{array}$ & $\begin{array}{l}\text { mean } \pm \\
\text { SD }(\%)\end{array}$ & $\begin{array}{l}\text { mean } \\
-2 \text { sd }\end{array}$ \\
\hline${ }^{82} R b R$ & $0.47 \pm 12$ & 0.36 & $0.82 \pm 15$ & 0.57 & $1.78 \pm 16$ & 1.19 \\
\hline${ }^{82} R b K_{l}$ & $0.48 \pm 18$ & 0.31 & $1.11 \pm 24$ & 0.58 & $2.35 \pm 27$ & 1.08 \\
\hline${ }^{82} R b K_{1}-D V$ & $0.46 \pm 19$ & 0.28 & $1.01 \pm 16$ & 0.68 & $2.26 \pm 23$ & 1.23 \\
\hline${ }^{82} R b K_{1}-D V$ const. & $0.45 \pm 19$ & 0.27 & $1.01 \pm 17$ & 0.67 & $2.34 \pm 24$ & 1.22 \\
\hline${ }^{13} \mathrm{NH}_{3} \mathrm{R}$ & $0.61 \pm 14$ & 0.44 & $1.32 \pm 17$ & 0.87 & $2.17 \pm 16$ & 1.46 \\
\hline${ }^{82} \mathrm{Rb} M B F_{R}$ & $0.71 \pm 26$ & 0.34 & $2.95 \pm 41$ & 0.55 & $4.26 \pm 39$ & 0.98 \\
\hline${ }^{82} R b M B F_{K 1}$ & $0.73 \pm 31$ & 0.28 & $2.96 \pm 37$ & 0.76 & $4.32 \pm 42$ & 0.69 \\
\hline${ }^{82} R b M B F_{K l^{-}} D V$ & $0.68 \pm 32$ & 0.25 & $2.54 \pm 25$ & 1.26 & $4.08 \pm 36$ & 1.16 \\
\hline${ }^{82} R b M B F_{K 1}-D V$ const. & $0.64 \pm 31$ & 0.25 & $2.53 \pm 26$ & 1.20 & $4.30 \pm 37$ & 1.09 \\
\hline${ }^{13} \mathrm{NH}_{3} M B F_{R}$ & $0.68 \pm 19$ & 0.43 & $2.86 \pm 43$ & 0.4 & $4.15 \pm 40$ & 0.83 \\
\hline${ }^{13} \mathrm{NH}_{3} \mathrm{MBF}-\mathrm{DV}$ & $0.65 \pm 22$ & 0.36 & $2.25 \pm 21$ & 1.30 & $3.64 \pm 29$ & 1.52 \\
\hline${ }^{13} \mathrm{NH}_{3} \mathrm{MBF}$ - DV const. & $0.61 \pm 19$ & 0.38 & $2.25 \pm 22$ & 1.26 & $3.79 \pm 27$ & 1.78 \\
\hline${ }^{13} \mathrm{NH}_{3} \mathrm{MBF}$ & $\mathbf{0 . 7 2} \pm \mathbf{2 0}$ & 0.43 & $2.81 \pm 27$ & 1.31 & $4.04 \pm 32$ & 1.48 \\
\hline
\end{tabular}

The normal ranges and the lower limits of normal (mean - 2SD) vary substantially between the models, as well as between the tracers, particularly in the regional databases. For both tracers, the net retention values had smaller normal ranges $(R$ mean $\mathrm{SD}=15 \%$ $[12-17 \%])$ than the corresponding compartment model uptake rates $\left(K_{I}\right.$ mean $\mathrm{SD}=23 \%$ [18-32\%]). The uptake rates from the one-compartment DV and constant-DV models had similar normal ranges to the net retention rate method ( $K_{1}-D V, K_{I}-D V$ const. mean $\mathrm{SD}=17 \%$ ). For ${ }^{13} \mathrm{NH}_{3}$, the normal flow ranges derived from retention $\left({ }^{13} \mathrm{NH}_{3} M B F_{R} \mathrm{SD}\right.$ $=34 \%[19-43 \%])$ were generally higher than those of the standard two-compartment model flow $\left({ }^{13} \mathrm{NH}_{3} \mathrm{MBF} \mathrm{SD}=26 \%\right.$ [20-32\%]), and the flow values from the onecompartment DV models $\left({ }^{13} \mathrm{NH}_{3} \mathrm{MBF}-\mathrm{DV},{ }^{13} \mathrm{NH}_{3} \mathrm{MBF}-\mathrm{DV}\right.$ const. SD $=23 \%[19-$ $29 \%]$ ), due to the non-linear extraction correction applied to the retention rates. In contrast for ${ }^{82} \mathrm{Rb}$, the normal ranges of flow derived from retention $\left({ }^{82} R b M B F_{R} \mathrm{SD}=\right.$ 
$35 \%$ [26-41\%]) showed minimal change compared to the compartment model-derived flows $\left({ }^{82} R b M B F_{K l},{ }^{82} R b M B F_{K l^{-}} D V,{ }^{82} R b M B F_{K l^{-}} D V\right.$ const. mean SD $=33 \%$ [25$42 \%]$ ), due to similarity in the respective extraction corrections. On average, the ${ }^{82} \mathrm{Rb}$ normal flow ranges $\left({ }^{82} R b M B F_{R},{ }^{82} R b M B F_{K 1},{ }^{82} R b M B F_{K l^{-}} D V,{ }^{82} R b M B F_{K l^{-}} D V\right.$ const. $\mathrm{SD}=34 \%$ ) were the same as the ${ }^{13} \mathrm{NH}_{3}$ retention-derived flow range $\left({ }^{13} \mathrm{NH}_{3} M B F_{R} \mathrm{SD}=\right.$ $34 \%)$, but were larger than the ${ }^{13} \mathrm{NH}_{3}$ compartmental flow range $\left({ }^{13} \mathrm{NH}_{3} M B F_{K 1} \mathrm{SD}=\right.$ $24 \%$ ). For both tracers, the one-compartment model DV variants exhibited smaller normal ranges and thus higher lower limits of normal flow than the other compartment models for both tracers. From these results, it can be concluded that the net retention model may have similar ability for detecting and localizing defects using ${ }^{82} \mathrm{Rb}$ and ${ }^{13} \mathrm{NH}_{3}$, prior to the application of an extraction correction to obtain absolute flow estimates, since this method demonstrates lower variability than the standard compartment models.

A visual comparison of the uniformity within the databases is provided in Figure 4.1. Polar maps for each database of the uptake rate or flow, depending on the specific database, are shown at rest, stress and reserve. From this perspective it is evident that the compartmental models show some improved uniformity at the apex due to the estimated partial volume correction that is incorporated into the method. However, the net retention rate method results in more uniform polar maps overall. 


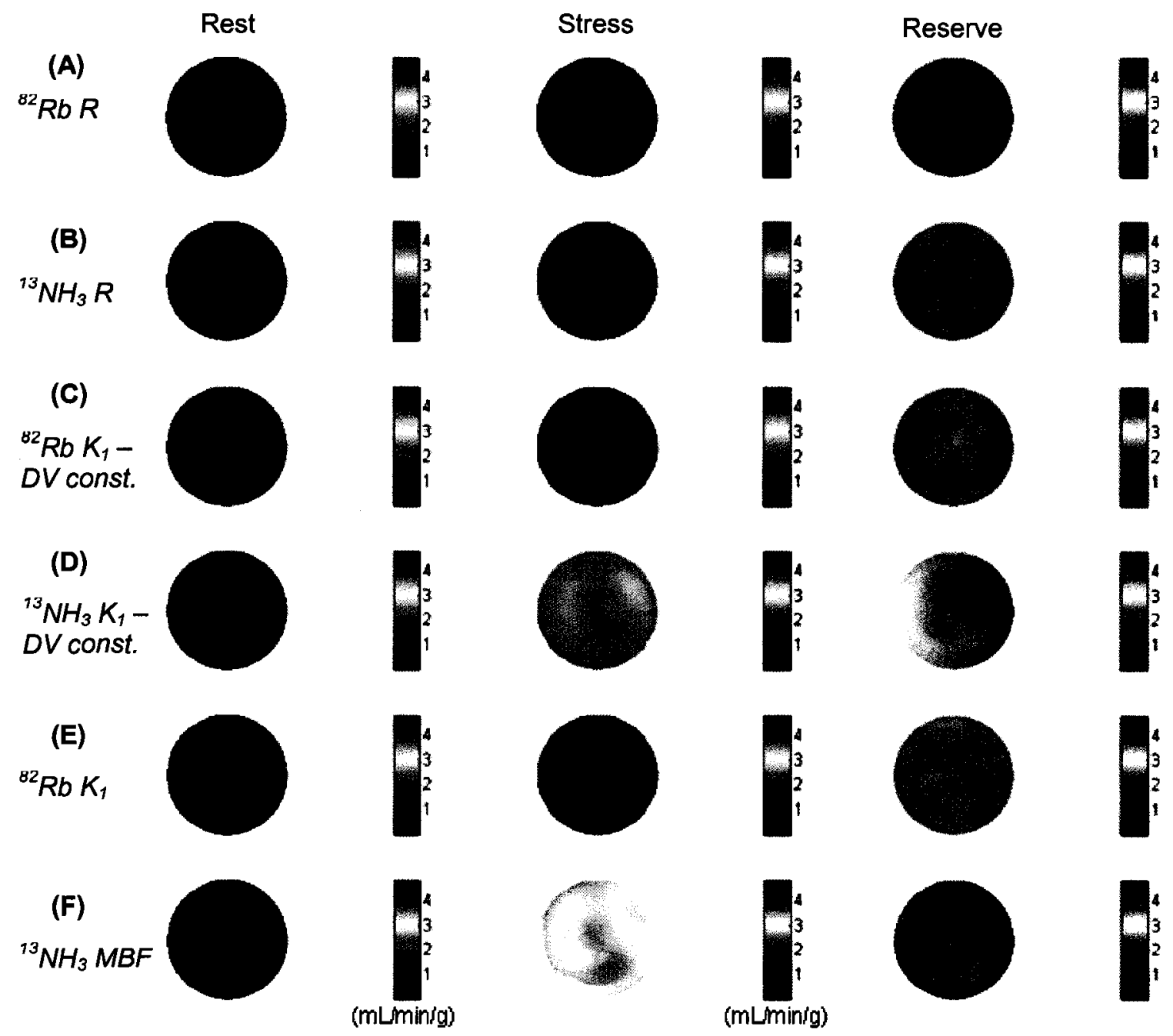

Figure 4.1: Regional polar maps of the normal population mean (A) ${ }^{82}$ Rb retention rate $(R),(B){ }^{13} \mathrm{NH}_{3}$ retention rate $(R),(C){ }^{82} \mathrm{Rb}$ one-compartment constant-DV $\left(K_{I}\right)$, (D) ${ }^{13} \mathrm{NH}_{3}$ one-compartment constant-DV $\left(K_{1}\right)$, (E) ${ }^{82} \mathbf{R b}$ one-compartment model $\left(K_{l}\right)$, and $(F){ }^{13} \mathrm{NH}_{3}$ two-compartment model $(M B F)$. Note that the normal apical reduction in tracer retention is normalized by the geometric partial volume correction included with the compartmental models; however, the compartmental models exhibit a wider normal range.

The normal MBF values are similar between the ${ }^{82} \mathrm{Rb}$ one-compartment and ${ }^{13} \mathrm{NH}_{3}$ twocompartment models; however, the MBF estimates obtained with the one-compartment DV and constant-DV models for both tracers appear to be lower in comparison. Regional estimates of $K_{l}$ generated by the ${ }^{82} \mathrm{Rb}$ one-compartment model and its DV variants 
provided a slightly reduced variability $\left(K_{l}\right.$ mean SD averaged over rest, stress and reserve decreased by $-5.6 \%$ ) compared to those for ${ }^{13} \mathrm{NH}_{3}$ from the standard two-compartment model $\left(K_{l}=M B F\right)$, yet the derived estimates of flow tended to be more variable with the one-compartment model ( $M B F$ mean SD increased $+10.3 \%)$, due to amplification of noise by the non-linear extraction correction. In contrast, the MBF variability was actually overall slightly lower with the DV models ( $M B F$ mean SD decreased by $-3 \%$ ); this is likely due to the increased model stability afforded by the use of the DV parameter. Similar results were observed for the DV models with ${ }^{13} \mathrm{NH}_{3}$. Therefore, it appears that the ${ }^{13} \mathrm{NH}_{3}$ two-compartment model still provides the most precise estimation of absolute normal MBF and MFR.

Based on these results, a more detailed analysis focused on the databases providing the smallest normal ranges and best lower limits of normal, including the: ${ }^{82} R b R,{ }^{82} R b$ $M B F_{R}{ }^{13} \mathrm{NH}_{3} \mathrm{R},{ }^{13} \mathrm{NH}_{3} \mathrm{MBF}_{\mathrm{R}}{ }^{82} \mathrm{Rb} \mathrm{K}_{1}-\mathrm{DV}$ constant, ${ }^{82} \mathrm{Rb} M B F_{K l}-\mathrm{DV}$ constant, and ${ }^{13} \mathrm{NH}_{3} \mathrm{MBF}-\mathrm{DV}$ constant databases. Analysis of the difference between the regional mean retention rate at rest, stress, and reserve, versus the flow values derived from the compartment models, was completed using $t$-tests, the results of which are shown in Figure 4.2. The comparison of the variability in the regional databases using $F$-tests is shown in Figure 4.3. 

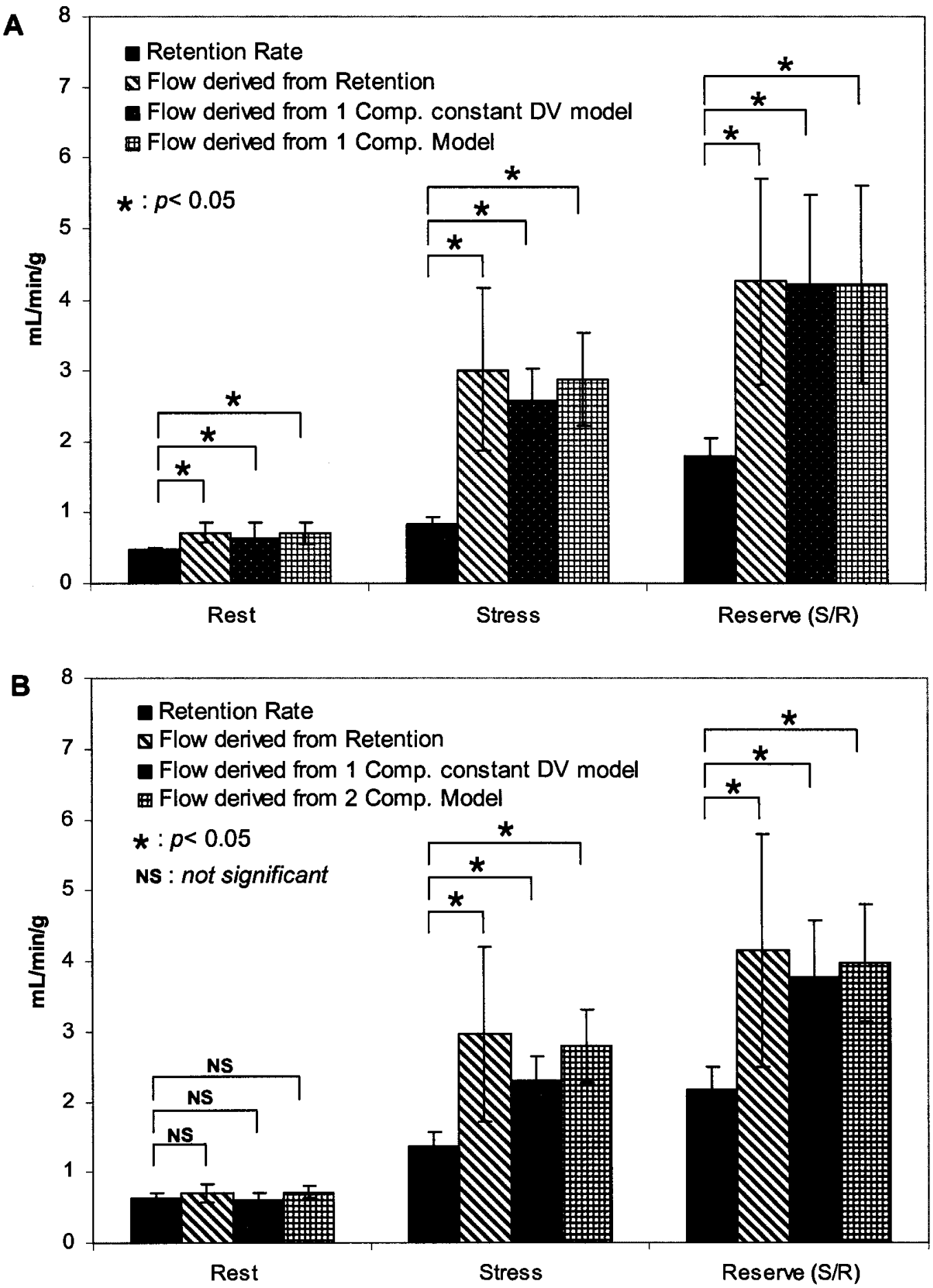

Figure 4.2: Comparison of the mean values from the retention and compartmental model databases for (A) ${ }^{82} \mathrm{Rb}$ and $(B){ }^{13} \mathrm{NH}_{3}$. 

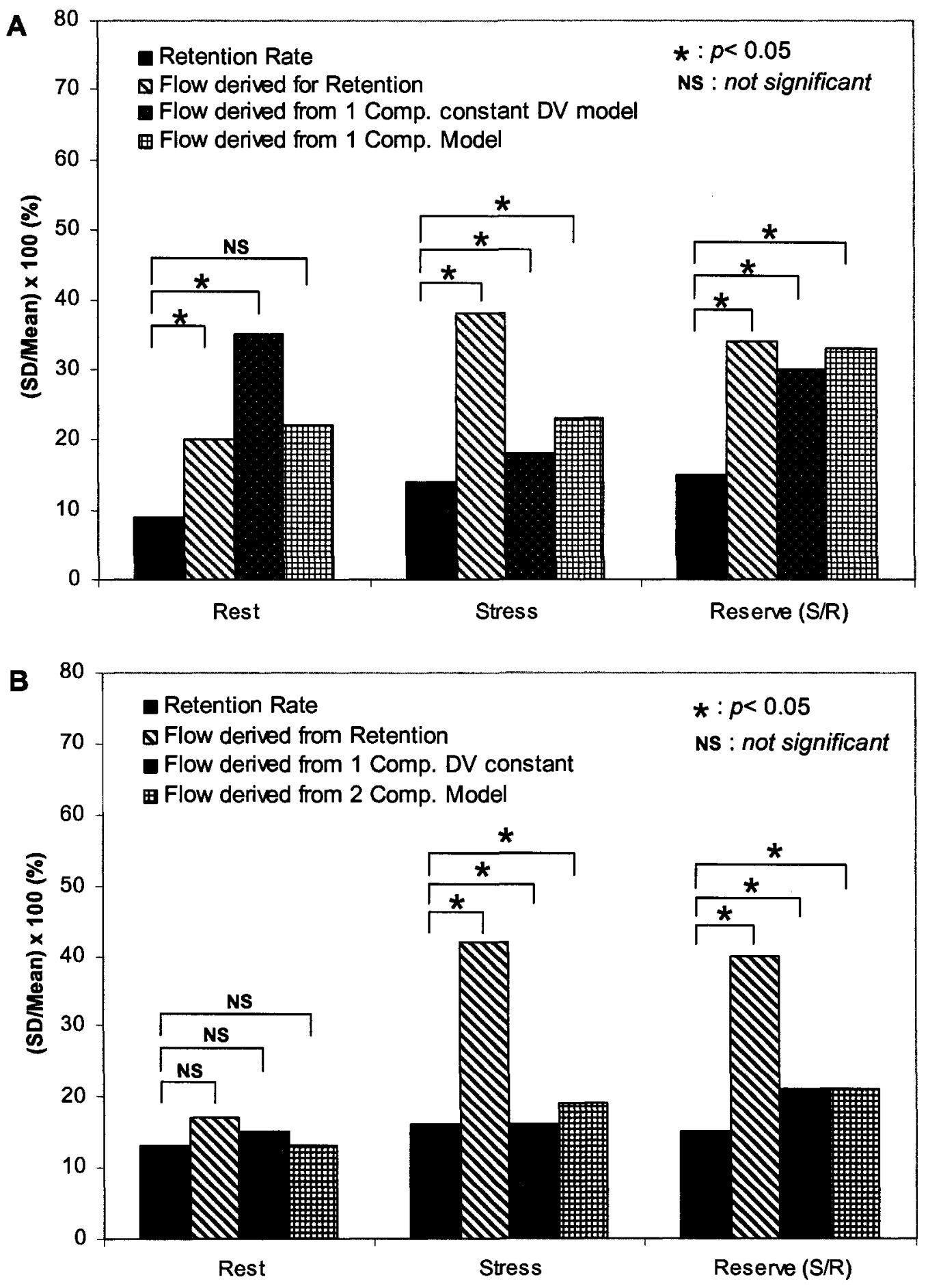

Figure 4.3: Comparison of the variability from the retention and compartmental model databases for $(A){ }^{82} \mathrm{Rb}$ and $(B){ }^{13} \mathrm{NH}_{3}$. 
By design, the flow values from the net retention method for ${ }^{82} \mathrm{Rb}$ are not significantly different than those obtained from the compartment models. However, the retention rate values and the uptake values $\left(K_{l}\right)$ are significantly lower than the derived flow values, as expected. Similar results were observed with ${ }^{13} \mathrm{NH}_{3}$, with the exception of the rest results where a significant difference was not noted between the retention rate and the flow estimates. This is presumably due to the high extraction of ${ }^{13} \mathrm{NH}_{3}$ from the blood into the tissue at low flow rates. The evaluation of the variability between the databases demonstrated a significantly lower variability with the retention rate method as compared to the flow-derived methods for both tracers. Again, this suggests that the retention rate may be preferable for the detection of CAD.

As an additional consideration, the precision on the SD measurements for each of the selected databases was assessed. This metric is representative of the regional uniformity of the database polar maps, which is an important criterion when selecting a database for routine clinical use. To determine the regional uniformity, the standard deviations of the SD polar maps for each database were calculated and normalized by the mean of the corresponding database, providing $\mathrm{COV}$ measurements that were indicative of the distribution of the SD measurements across the polar maps. The results of this analysis are expressed as percentages in Table 4.3. The most useful clinical metrics are the stress and reserve values; thus, considering the stress and reserve flow parameters $\left({ }^{82} R b M B F_{R}\right.$, ${ }^{82} \mathrm{Rb} M B F_{K 1}-D V$ constant, ${ }^{13} \mathrm{NH}_{3} M B F_{R},{ }^{13} \mathrm{NH}_{3} \mathrm{MBF}-\mathrm{DV}$ constant) it is evident that they are much less uniform across the polar map than the uptake databases $\left({ }^{82} R b R,{ }^{82} R b\right.$ $K_{1}-D V$ constant, $\left.{ }^{13} \mathrm{NH}_{3} \mathrm{R}\right)$. This is due to the extraction correction that has been applied 
to obtain the flow values, which adds a large amount of variability to the regional database polar maps. This may suggest that the MBF databases formed with tracer extraction corrections are not suitable for localizing disease because the uniformity, and hence the regional lower limits of normal, are quite variable.

\section{Table 4.3: Regional uniformity of the database polar maps}

\begin{tabular}{|c|c|c|c|}
\hline \multirow[t]{2}{*}{ Database } & \multicolumn{3}{|c|}{$\begin{array}{l}\text { Standard Deviation of SD polar-map / Mean } * 100 \\
(\% \text { COV })\end{array}$} \\
\hline & Rest & Stress & Reserve \\
\hline${ }^{82} R b R$ & 1 & 3 & 5 \\
\hline${ }^{82} R b K_{1}-D V$ const. & 5 & 7 & 12 \\
\hline${ }^{13} \mathrm{NH}_{3} \mathrm{R}$ & 2 & 5 & 7 \\
\hline${ }^{82} R b M B F_{R}$ & 7 & 38 & 39 \\
\hline${ }^{82} R b M B F_{K l}-D V$ const. & 16 & 28 & 38 \\
\hline${ }^{13} \mathrm{NH}_{3} M B F_{R}$ & 5 & 56 & 49 \\
\hline${ }^{13} \mathrm{NH}_{3} \mathrm{MBF}-\mathrm{DV}$ const. & 4 & 16 & 24 \\
\hline
\end{tabular}

To determine the precision of the SD polar maps from a sample of healthy volunteers as used in this study $(\mathrm{N}=14)$, the variance of a normal distribution was considered. This was done to determine how precise the SD estimates were for a given population size to obtain an indication of the relevance of the sample size of 14 used in this study. To generate the estimates, random numbers, $x$, from a standard normal distribution with a mean, $\mu$, of zero and a variance, $\sigma^{2}$, of 1 , characterized by the probability density function expressed in Eq. 4.1, were used [58].

$$
f(x ; 0,1)=\frac{1}{\sqrt{2 \pi}} e^{-x^{2} / 2}
$$

This distribution was considered appropriate for comparison with the population of healthy subjects based on the postulate of the central limit theorem, which states that if a 
sample size is sufficiently large then the mean of the sample tends to the normal distribution [58]. In this case it was assumed that even if the recruited population was not normally distributed, the mean values of the sample would be, thus making it appropriate to compare those values to the ones generated for a standard normally distributed population. Sample sizes varying from 14 to 2500 , consisting of 10,000 sample sets were generated. The standard deviations of the resultant distributions were subsequently computed to obtain measures of precision. The results of this analysis are expressed as SD percentages in Table 4.4 .

\section{Table 4.4: Sample standard deviation of random normal distributions}

\begin{tabular}{cc}
\hline Sample size & SD\% \\
\hline 14 & 19 \\
20 & 16 \\
25 & 15 \\
50 & 10 \\
100 & 7 \\
200 & 5 \\
300 & 4 \\
1000 & 2 \\
2500 & 1 \\
\hline
\end{tabular}

For a sample size of 14 , the random normal distribution has a better precision than that determined for all of the flow databases using the SD polar maps. Again, this can be explained by the application of the extraction corrections causing greater non-uniformity than would be observed in a normal randomly distributed sample. In comparing the results for the sample sizes of 100 and 200 (Table 4.4) with the SD polar map results for the ${ }^{13} \mathrm{NH}_{3}$ and ${ }^{82} \mathrm{Rb}$ net retention rate databases (Table 4.3), it can be interpreted that these databases have uniformities that would be equivalent to having sample sizes of 100 
and 200 , respectively, since the reserve $\% \mathrm{COV}$ values $(5 \%, 7 \%)$ match those of the SD $\%$ for the random normal distribution. This is due to the low population and regional variability in the retention databases for both tracers $\left({ }^{82} R b R\right.$ and ${ }^{13} \mathrm{NH}_{3} R$ in Table 4.2). Consequently, these results further support the finding that the net retention rate databases have an overall greater uniformity than the flow-derived databases.

\subsection{Discussion}

Normal population databases were developed representing the normal range of myocardial blood flow and MFR, measured using ${ }^{82} \mathrm{Rb}$ and ${ }^{13} \mathrm{NH}_{3}$, with compartmental modeling and a simple net retention model. The absolute flow values derived using the retention method, were not significantly different than those obtained from the compartment models. However, the retention rate values and the uptake rate values $\left(K_{l}\right)$ were significantly lower than the derived flow estimates, as expected, but also demonstrated significantly lower variability. The study suggested that the detection and localization of absolute flow reductions should be best achieved using ${ }^{13} \mathrm{NH}_{3}$ with compartment modeling, since it yields the smallest normal flow range (SD\%), both globally and regionally.

However, it is important to note that detection and localization of disease do not require absolute flow quantification, a-priori. This study demonstrated that the net retention rate (R) consistently decreases the measured normal range at rest, stress and stress/rest compared to the compartmental model uptake rate $\left(K_{1}\right)$ for both ${ }^{82} \mathrm{Rb}$ and ${ }^{13} \mathrm{NH}_{3}$. The 
retention model had the lowest global SD\% values $(9-16 \%)$, indicating that this model should be best suited for the initial detection of global flow reductions in patients with CAD. Furthermore, the normal range of $R$ was almost equivalent for the regional measurements (+1-3\% vs. global), whereas the regional range of $K_{l}$ was increased (+6$11 \%$ vs. global). This was further demonstrated by the evaluation of the precision of the SD measurements, which resulted in the highest regional uniformity for the retention rate databases, suggesting that the net retention model is also advantageous for disease localization. Together, these results suggest that the net retention model is optimal for the establishment of an absolute scale normal database for both ${ }^{82} \mathrm{Rb}$ and ${ }^{13} \mathrm{NH}_{3}$ due to its decreased population variability and improved regional uniformity over the compartmental models.

Figure 4.4 shows an example of the ${ }^{13} \mathrm{NH}_{3}$ net retention rate $\left({ }^{13} \mathrm{NH}_{3} R\right.$ in $\left.4.4 \mathrm{~A}, \mathrm{~B}\right)$ database compared to the derived flow database $\left({ }^{13} \mathrm{NH}_{3} M B F_{R}\right.$ in $\left.4.4 \mathrm{C}\right)$ applied to the polar maps of a patient to detect and localize CAD. The ${ }^{l 3} \mathrm{NH}_{3} \mathrm{R}$ database identifies a much larger abnormal region (dark blue on stress and reserve (stress/rest) imaging) than the derived flow database $(34.86 \%$ of LV vs. $8.98 \%$ of LV), demonstrating improved disease detection. In addition, the retention-derived flow scale $\left(\mathrm{MBF}_{\mathrm{R}}\right)$ has been added to the retention colour scale in Figure 4.4 B, showing the thresholds of abnormal stress flow $\left(\mathrm{MBF}_{\mathrm{R}}<1.5 \mathrm{~mL} / \mathrm{min} / \mathrm{g}\right)$ and flow reserve (stress/rest $\left.<2.3\right)$ that correspond with the normal limits of net retention. This derived normal limit of flow reserve is markedly improved (higher) compared to the regional normal value obtained with the ${ }^{13} \mathrm{NH}_{3}$ compartment model (stress/rest $<1.48$ ), suggesting again that the net retention method 
should be more sensitive for disease detection. To validate this observation, further studies in patients with $\mathrm{CAD}$ were required to determine the most appropriate thresholds for disease detection and to assess the accuracy of disease detection and localization using this method, as discussed in Chapter 5.

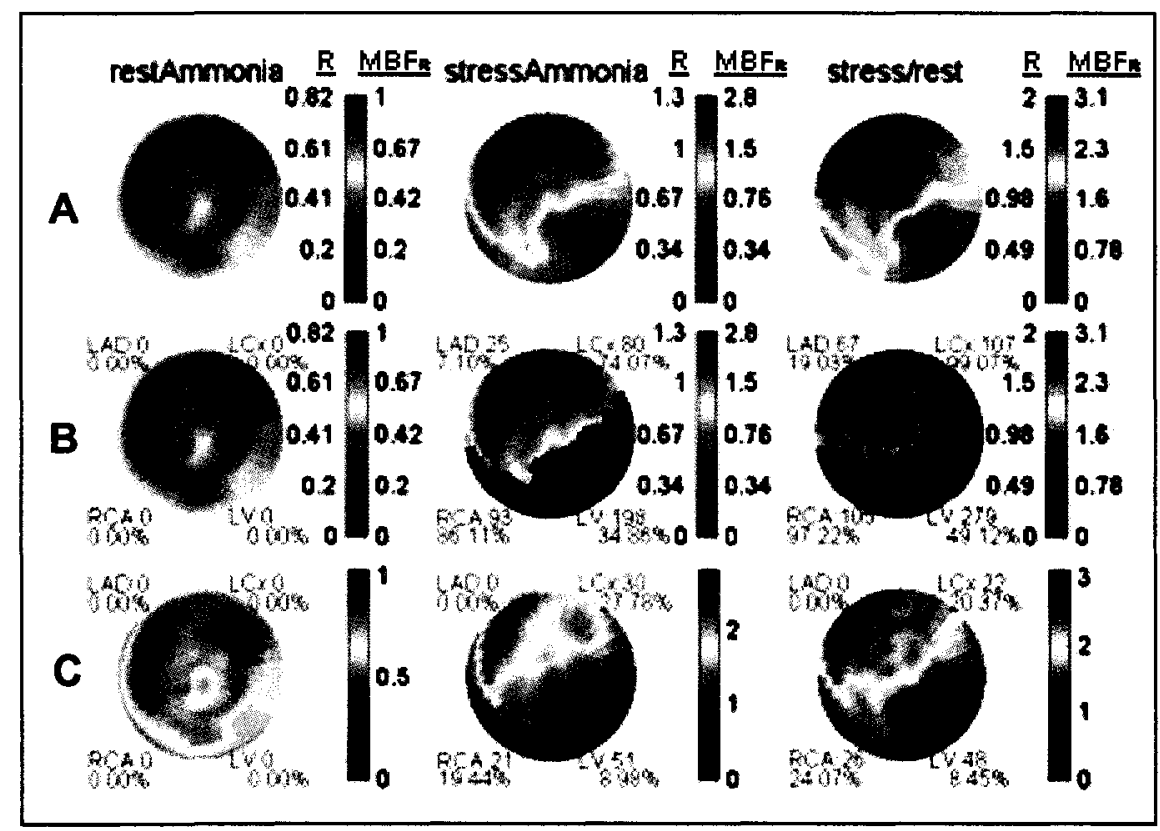

Figure 4.4: Rest, stress and stress/rest polar maps in a subject with CAD. (A) ${ }^{13} \mathrm{NH}_{3}$ net retention rate $\left({ }^{13} \mathrm{NH}_{3} \mathrm{R}\right)$. (B) and (C) show the abnormal sector maps (dark blue) for the ${ }^{13} \mathrm{NH}_{3} \mathrm{R}$ and the derived flow values $\left({ }^{13} \mathrm{NH}_{3} M B F_{R}\right)$ more than $2 \mathrm{SDs}$ below the regional normal population mean. The decreased variability of the ${ }^{13} \mathrm{NH}_{3} R$ database results in the identification a larger abnormal region as compared to the ${ }^{13} \mathrm{NH}_{3} \mathrm{MBF}_{\mathrm{R}}$ database, indicating that the ${ }^{13} \mathrm{NH}_{3} \mathrm{R}$ database is more sensitive for detection and localization of absolute flow reductions. 


\section{CHAPTER 5 - Evaluation of Patients Using Normal Population Databases}

\subsection{Characterization of the Patient Population}

To evaluate the performance of the normal population databases, the normal limits and criteria for detection of MBF abnormalities were validated in a preliminary study consisting of a small group of 26 patients. This group included two sub-populations: a low-likelihood of disease (normal) population and a population with known CAD, as documented by coronary angiography. The normal group consisted of 14 subjects ( 7 men, 7 women; $20-45$ years old; mean \pm SD age $=34 \pm 7$ years), and the CAD group included 12 subjects (10 men, 2 women; $41-80$ years old; mean \pm SD age $=62 \pm 14$ years). Note that this group of normal subjects was independent from the population that was used for the formation of the normal databases, and the $\mathrm{CAD}$ group was not the same cohort that was used for the validation of the extraction functions.

Both groups underwent rest and stress dynamic ${ }^{82} \mathrm{Rb}$ imaging. Scans were acquired on the ECAT ART PET system following the protocol listed in Chapter 1, section 1.3. Images were reconstructed and processed according to the methods described in Chapter 1, sections 1.4 and 1.5. The retention and one-compartment constant-DV models were applied to the processed data for each subject to obtain retention rate, uptake rate $\left(K_{I}\right)$ and MBF values. Clinical angiography reports were used as a truth standard for comparison with the PET findings for the CAD group. The threshold for a significant stenosis 
(narrowing) in one or more of the major coronary arteries was defined as $70 \%$ or greater in diameter. The regional and global results of abnormality were organized in a truth table of actually-positive cases (areas with disease) and actually-negative cases (areas without disease) to facilitate comparison with the PET findings. To determine if the net retention rate was indeed the optimal method for use in relative and absolute databases for detection and localization of disease in patients, receiver operating characteristics (ROC) were evaluated for the models listed.

\subsection{Receiver Operating Characteristic Analysis}

The common metric used to assess the accuracy of any diagnostic test is the ROC technique. An ROC curve is a plot of the sensitivity (SEN), or true positive rate (TPR), versus 1 - specificity (SPC), or the false positive rate (FPR), of a method. The sensitivity and specificity are defined as follows:

$$
\begin{aligned}
& S E N=T P R=\frac{T P}{T P+F N} \\
& S P C=1-F P R=\frac{T N}{F P+T N}
\end{aligned}
$$

where TP is the number of true positive results, i.e. the number of regions that were identified to be abnormal and were truly abnormal; TN represents the true negative results, i.e. regions that were identified to be normal and were truly normal; false positive results, denoted by FP, are regions that were identified to be abnormal but were truly normal; and false negative results, FN, are regions that were truly abnormal but were 
identified as normal. Each point on the ROC curve represents a specific sensitivity/specificity pair, referred to as an operating point. By fitting a curve to the operating points, the dynamics of how the TPR and the FPR vary together can be visualized. Examples of typical ROC curves are displayed in Figure 5.1. A diagnostic test with perfect accuracy will have $100 \%$ sensitivity at each FPR, indicating that the test does not generate any false positive or false negative results. A moderately accurate test will have a monotonically increasing sensitivity that rises with the FPR values until reaching $100 \%$. A test that generates a point along the chance diagonal line indicates that the diagnostic method is unable to discriminate between presence and absence of disease and is hence not very useful; a test with an ROC curve of this nature would be equivalent to guessing a diagnosis. The chance diagonal line divides the ROC space into areas of relatively accurate versus inaccurate diagnostic abilities. Points above the diagonal line indicate useful results, while points below the line indicate relatively false results. The use of ROC curves provides a complete assessment of the performance of a diagnostic test over a range of decision-making thresholds, rather than determining the sensitivity and specificity for a single cutoff. However, it is often of use to determine a particular operating point, describing the optimal cutoff to use for achieving the maximal sensitivity and/or specificity when applying a given method; for example, a diagnostic (rule-in) test requires an operating point with high sensitivity, whereas a screening (rule-out) test necessitates high specificity. 


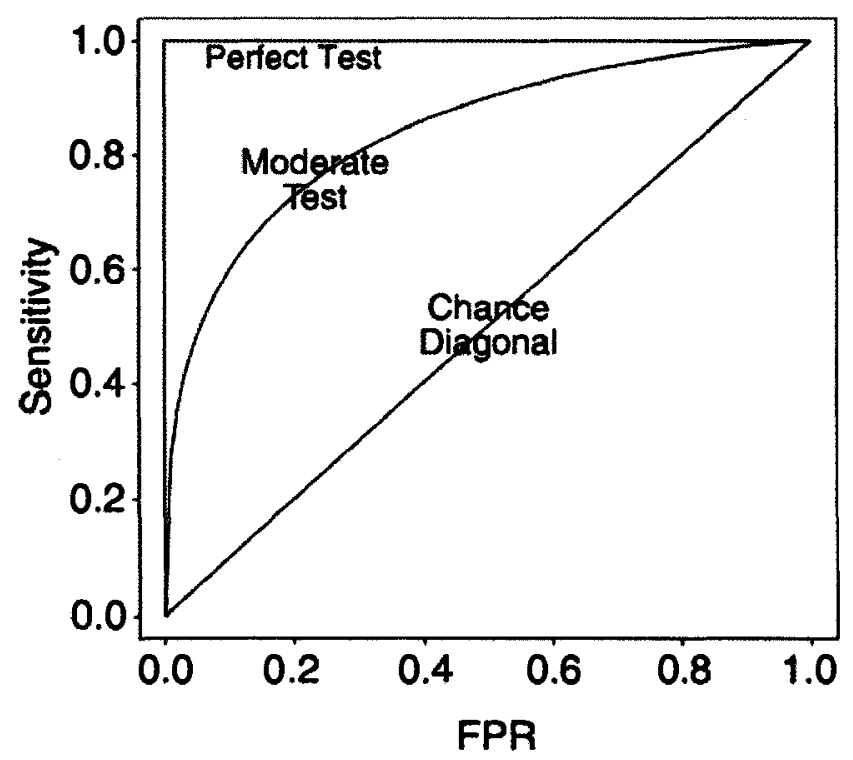

Figure 5.1: ROC curves for a perfectly accurate diagnostic test, a moderately accurate test, and the chance diagonal representing a test that is unable to discriminate between healthy and diseased states [59].

ROC analysis was performed for the model databases on a relative scale to determine if the sensitivity and specificity results compared to the values reported in literature for ${ }^{82} \mathrm{Rb}$ relative MPI databases. The relative analysis was complemented by an evaluation of the databases on an absolute scale to determine the incremental value of the absolute quantification method in detecting or localizing disease, which has not been fully characterized in any previously reported studies.

As this was a retrospective group, the study at the time was not designed to include ${ }^{13} \mathrm{NH}_{3}$ imaging; hence the analysis in this chapter is limited to evaluation of the ${ }^{82} \mathrm{Rb}$ databases only. For each patient in the CAD population, the retention rate, uptake rate $\left(K_{l}\right)$ and flow values at stress were compared with the corresponding ${ }^{82} \mathrm{Rb}$ database parameters at SD 
thresholds of $1.5,2,2.5$ and 3 . Note that only the stress values were used since the stress scan is typically interpreted to diagnose CAD. The resultant percent of LV myocardium with abnormal sectors at each threshold level was termed the defect size. The defect sizes for each parameter were determined globally (i.e. over the whole LV), as well as regionally for the three territories corresponding to the major coronary arteries: the left anterior descending coronary artery (LAD), the left circumflex coronary artery (LCX) and the right coronary artery (RCA) regions (Fig. 5.2). For the global analysis, the data from the normal population was also included to generate specificity values, as calculation of this parameter requires knowledge of the TN fraction.

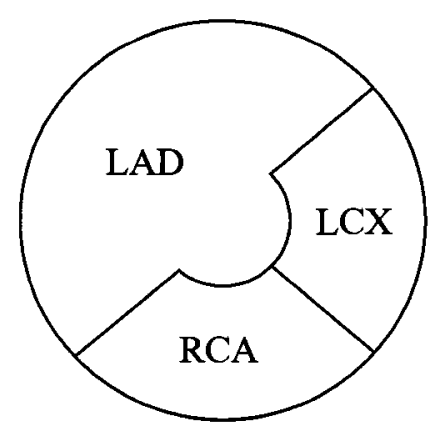

Figure 5.2: Division of the polar map into three vascular territories: LAD, LCX, and RCA.

By comparing the defect sizes obtained at the different SD thresholds to the truth table of coronary angiography findings, defining the actually-positive and actually-negative cases for each region and over the entire $L V$, sensitivities and specificities can be determined and used to perform ROC analysis. Using the global and regional data for each SD threshold, ROC curves were generated for global and regional assessment with the ROCKIT program from the University of Chicago [60]. Binormal ROC curves were fit to 
the percent defect size data from the whole $L V$, as well as the three territories, by calculating maximum-likelihood estimates of the parameters of a conventional binormal model for the input data. This model assumes that two normal probability densities form the basis of the ROC curve: one for actually-negative cases $(f(x \mid n))$, and one for actuallypositive $(f(x \mid p))$ cases [61]:

$$
\begin{aligned}
& f(x \mid n)=\frac{1}{\sqrt{2 \pi}} e^{-x^{2} / 2} \\
& f(x \mid p)=\frac{b}{\sqrt{2 \pi}} e^{-(b x-a)^{2} / 2}
\end{aligned}
$$

$f(x \mid n)$ has a mean of zero and an SD of 1 , and $f(x \mid p)$ has a mean of $a / b$ and an SD of $1 / b$, where $a$ and $b$ are the parameters that determine the form of the ROC curve. The variable $x$ is the decision variable. The FPR and TPR of a point on the ROC curve are given by the probability that the outcome of $x$ from actually-negative and actually-positive trials is greater than a critical value $x_{c}$, which is the cutoff value of a particular point on the ROC curve. The FPR and TPR for a particular point are thus expressed as:

$$
\begin{aligned}
& \operatorname{FPR}\left(x_{c}\right)=\Phi\left(-x_{c}\right) \\
& \operatorname{TPR}\left(x_{c}\right)=\Phi\left(a-b x_{c}\right)
\end{aligned}
$$

where $\Phi$ represents the standard normal distribution function [61]. Input data was automatically organized to choose percent defect size cutoffs for each SD threshold, thereby generating a useful spread of operating points on each ROC curve [62].

For each database, the area under the ROC curve at each SD threshold was tabulated for the whole LV and the three vascular territories, according to [61]: 


$$
A_{z}=\Phi\left(\frac{a}{\sqrt{(1+b)^{2}}}\right)
$$

The resultant area under the curve (AUC) values were plotted versus the corresponding SD thresholds for the relative and absolute databases, as shown in Figures 5.3 and 5.4, respectively. Note that the results for the flow derived from retention database $\left({ }^{82} \mathrm{Rb}\right.$ $\mathrm{MBF}_{\mathrm{R}}$ ) are not shown. This is due to the fact that ROC curves, and consequently the AUC values, could not be generated for this database as the dataset was deemed to be degenerate, meaning that there was not a large enough spread in the defect size data to determine relevant operating points to fit an ROC curve. This is due to large variability associated with this database, which prevents it from correctly detecting or localizing any significant number of defects at any of the SD thresholds. Also, for certain databases at the extremes of the SD thresholds, mainly 1.5 and 3 SDs, an AUC vs. SD point is not shown as the data was found to be similarly degenerate in these cases. This has been reported to be a problem for fitting a conventional binormal model to datasets containing only a small number of test results, or in cases where the data cannot be divided into a large enough number of categories and the categories are distributed over a small range [61]. 

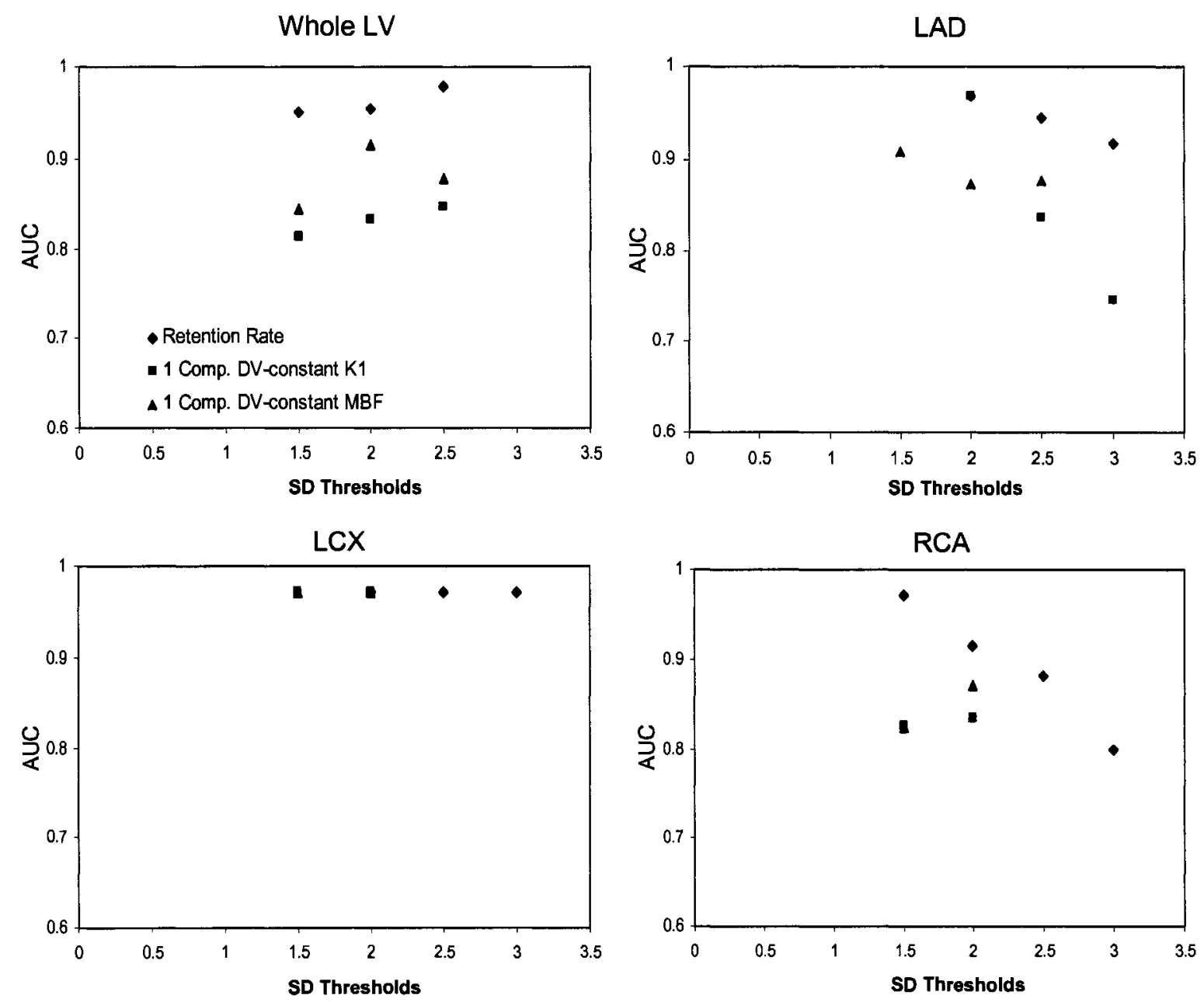

Figure 5.3: Whole LV and regional AUC versus SD threshold values for the relative ${ }^{82} \mathrm{Rb}$ databases. The net retention model consistently demonstrates the highest AUC values. 

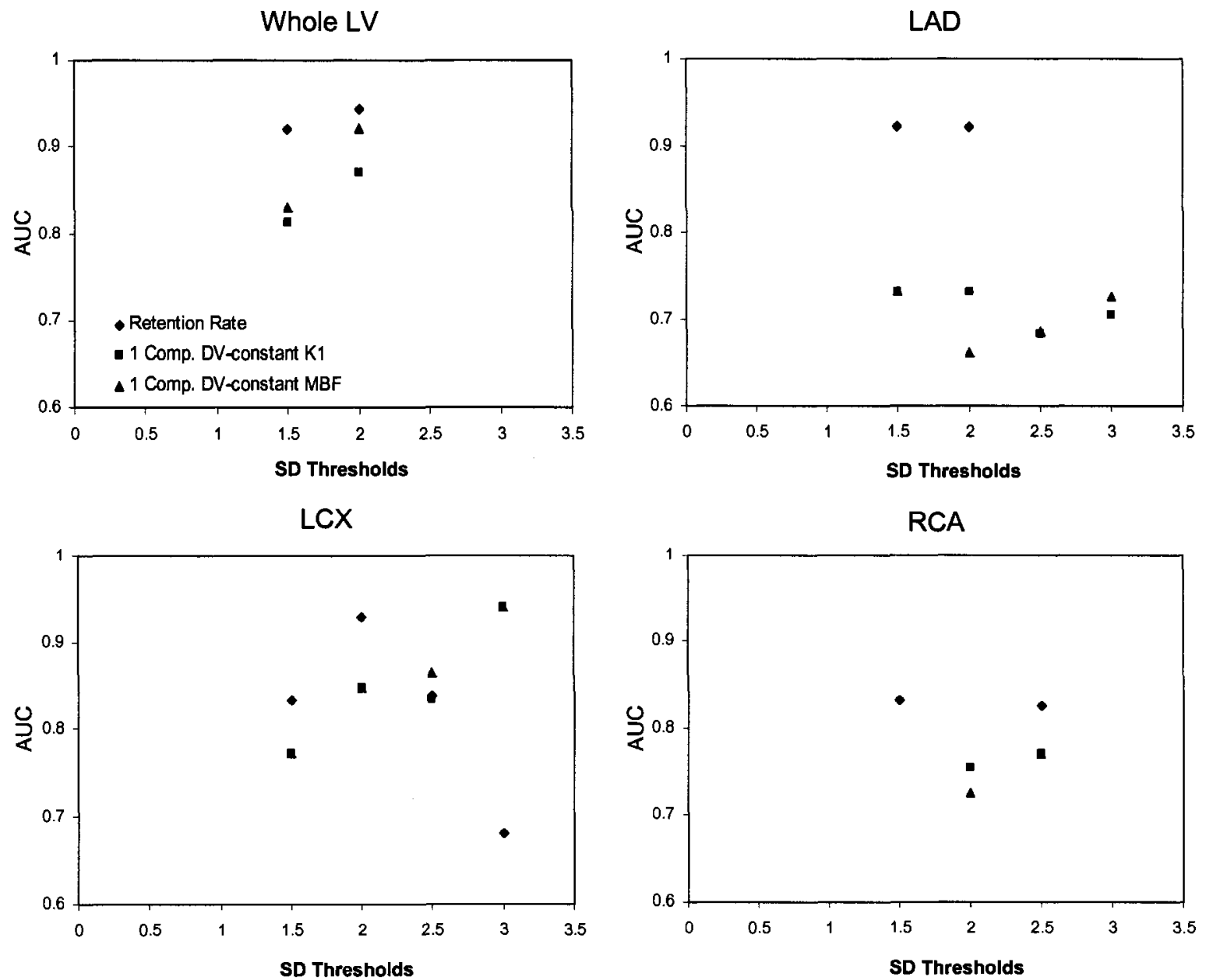

Figure 5.4: Whole $L V$ and regional AUC versus SD threshold values for the absolute ${ }^{82} \mathrm{Rb}$ databases. The net retention model generally has the highest AUC values. 
The databases that generated the highest AUC values were selected both globally and regionally, since tests with larger AUCs have higher overall accuracy. For the relative databases (Fig. 5.3), the retention method provided the largest AUC values on both global and regional levels, with SD thresholds of: 2.5 (whole LV), 2 (LAD), 2 (LCX), and 1.5 (RCA). On an absolute scale (Fig. 5.4), again the retention database generally gave the largest AUC values globally and regionally, with SD thresholds of: 2 (whole LV), 2 (LAD), 2.5 (LCX), and 1.5 (RCA). Note that for the LCX region, the one-compartment constant-DV $K_{1}$ method provided a slightly higher AUC value at an SD of 3 than the retention model at an $\mathrm{SD}$ of 2; however, upon further examination of the set of ROC curves from the one-compartment constant-DV $K_{l}$ database in the LCX region, the fitting was deemed to be suspect as the curves did not follow the expected trade-off pattern of decreasing sensitivity versus specificity with increasing SD threshold, as displayed in Figure 5.5. Similar investigation of the ROC curves for the retention model in the LCX region at SD thresholds of 1.5 and 2 showed uncharacteristic curve shapes, indicating that the fitting algorithm likely had difficulties in converging to an appropriate solution. Thus, while the AUC was largest with the retention model at an SD threshold of 2, the threshold of 2.5 was selected as the ROC curve fit at that cutoff appeared to be more reliable. 


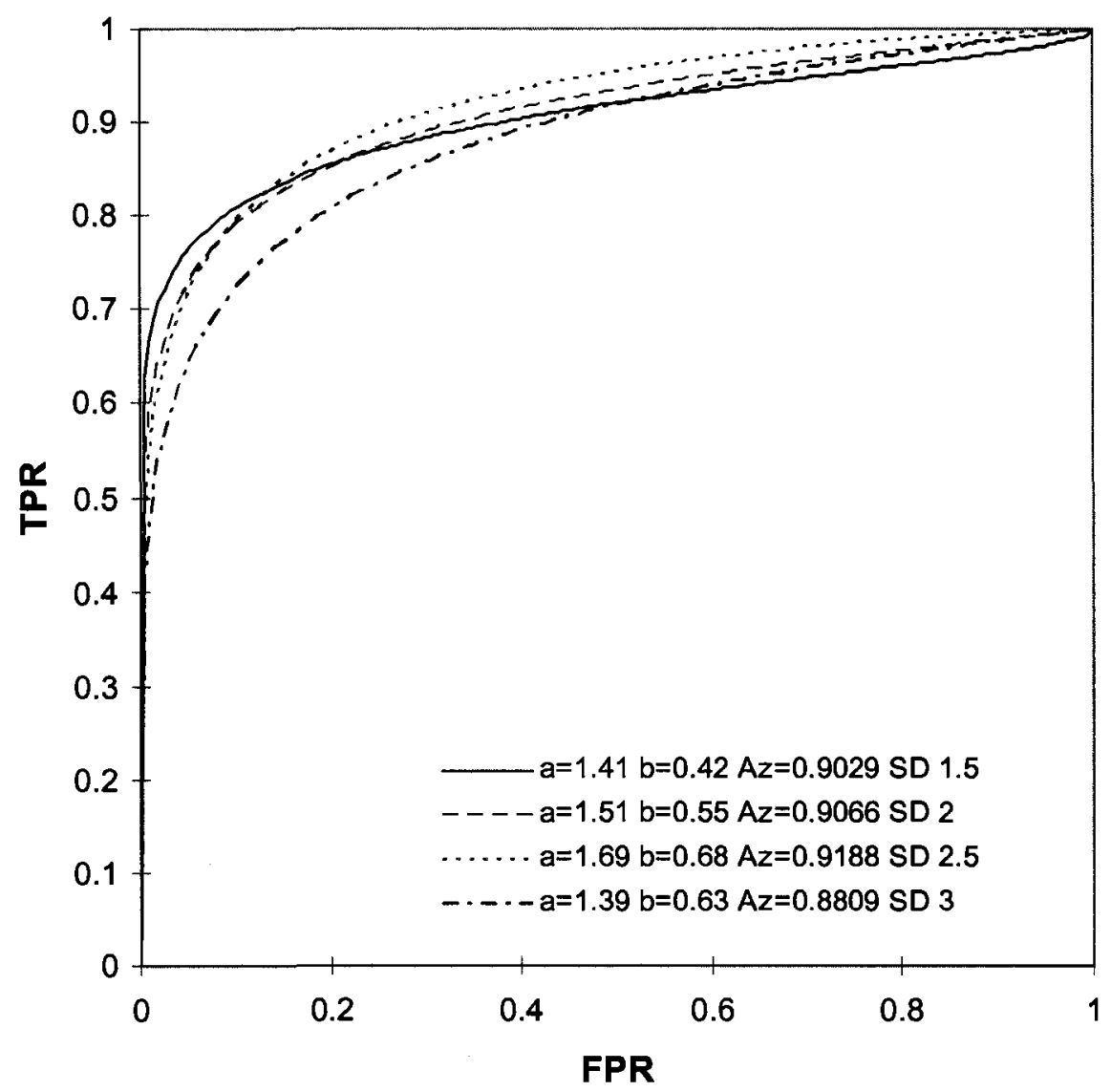

Figure 5.5: Characteristic pattern of regional ROC curves at varying SD thresholds. As the SD threshold changes, the trade-off between the sensitivity and specificity is altered.

From these results it is apparent that the retention database is the most effective for disease detection and localization as previously hypothesized, with SD thresholds ranging from 1.5 to 2.5 SDs. These results also suggest that the optimal sensitivity and specificity are obtained using different SD thresholds in each region. However, it is evident from the plots of AUC versus SD threshold that in many cases there is not a large difference between the AUCs at the varying thresholds. Furthermore, for ease of interpretation when applying the databases to evaluate patient scans in routine clinical application, it would 
be most useful to have one SD threshold level for all regions. This would prevent discrepancies in interpretation at the regional boundaries of neighbouring territories where differing extents of disease may be detected using inconsistent SD thresholds. For the relative retention database, the regional SD threshold can be selected at 2 SDs as it is the threshold with the highest AUC in most regions. If 2 SDs are selected rather than the region-specific SD thresholds, this will not have a large impact on the resultant sensitivity and specificity. For example, changing the SD threshold to 2 from 1.5 in the RCA region of the absolute database results in a change in AUC of $-5.5 \%$; this may or may not be an insignificant loss of accuracy, but cannot be fully evaluated here due to the small sample size. Globally however, while changing the SD threshold to 2 from 2.5 would only result in a AUC reduction of $2.5 \%$, the optimal SD threshold of 2.5 should be used to maintain the highest sensitivity for disease detection, as detection is the first and foremost goal; if disease cannot be detected, localization to one of the three territories becomes a null point.

For the absolute retention databases, an SD threshold of 2 was chosen for the LAD region, as well as for the whole LV, since the AUCs were highest at this threshold. For the RCA region, an SD threshold matching the global LV and LAD could not be selected as an ROC curve was not measurable at an SD of 2 . Additionally, in the LCX region, the ROC curve fits at SDs of 1.5 and 2 did not follow the expected pattern demonstrated in Figure 5.5. Therefore, for consistency between the RCA and LCX regions, an SD threshold of 2.5 was selected. Changing the SD threshold to 2.5 from the optimal value of 1.5 in the RCA region of the absolute database results in an AUC reduction of only $0.7 \%$. 
Thus, these SD threshold selections maintain the high level of accuracy for detection of disease, but may complicate interpretation at the regional boundaries due to the varying regional SD thresholds.

The regional and whole LV ROC curves corresponding to the chosen SD values were then used to determine the optimal operating points globally and regionally, representing the optimal SD threshold and percent of minimum defect for use in evaluating patients with the database. The operating point was selected by determining the percent defect size cutoff with the highest average sensitivity and specificity. The resultant minimum percent defect sizes with their corresponding sensitivities and specificities are given in Tables 5.1 and 5.2. The corresponding ROC curves are shown in Figures 5.6 and 5.7.

Table 5.1: SD thresholds and minimum percent defect size cutoffs with corresponding sensitivity and specificity for each region using the relative retention database

\begin{tabular}{lccccc}
\hline Region & $\begin{array}{c}\text { SD } \\
\text { threshold }\end{array}$ & $\begin{array}{c}\text { \% defect size } \\
\text { cutoff }\end{array}$ & $\begin{array}{c}\text { SEN } \\
(\%)\end{array}$ & $\begin{array}{c}\text { SPC } \\
(\%)\end{array}$ & $\begin{array}{c}\text { Mean (SEN, SPC) } \\
(\%)\end{array}$ \\
\hline Whole LV & 2.5 & 7 & 92 & 93 & 92 \\
LAD & 2 & 24 & 92 & 90 & 91 \\
LCX & 2 & 37 & 95 & 87 & 91 \\
RCA & 2 & 28 & 73 & 99 & 86 \\
\hline
\end{tabular}


Table 5.2: SD thresholds and minimum percent defect size cutoffs with corresponding sensitivity and specificity for each region using the absolute retention database

\begin{tabular}{lccccc}
\hline Region & $\begin{array}{c}\text { SD } \\
\text { threshold }\end{array}$ & $\begin{array}{c}\text { \% defect size } \\
\text { cutoff }\end{array}$ & $\begin{array}{c}\text { SEN } \\
(\%)\end{array}$ & $\begin{array}{c}\text { SPC } \\
(\%)\end{array}$ & $\begin{array}{c}\text { Mean (SEN, SPC) } \\
(\%)\end{array}$ \\
\hline Whole LV & 2 & 2 & 91 & 81 & 86 \\
LAD & 2 & 9 & 80 & 89 & 84 \\
LCX & 2.5 & 3 & 77 & 74 & 76 \\
RCA & 2.5 & 6 & 79 & 70 & 74 \\
\hline
\end{tabular}

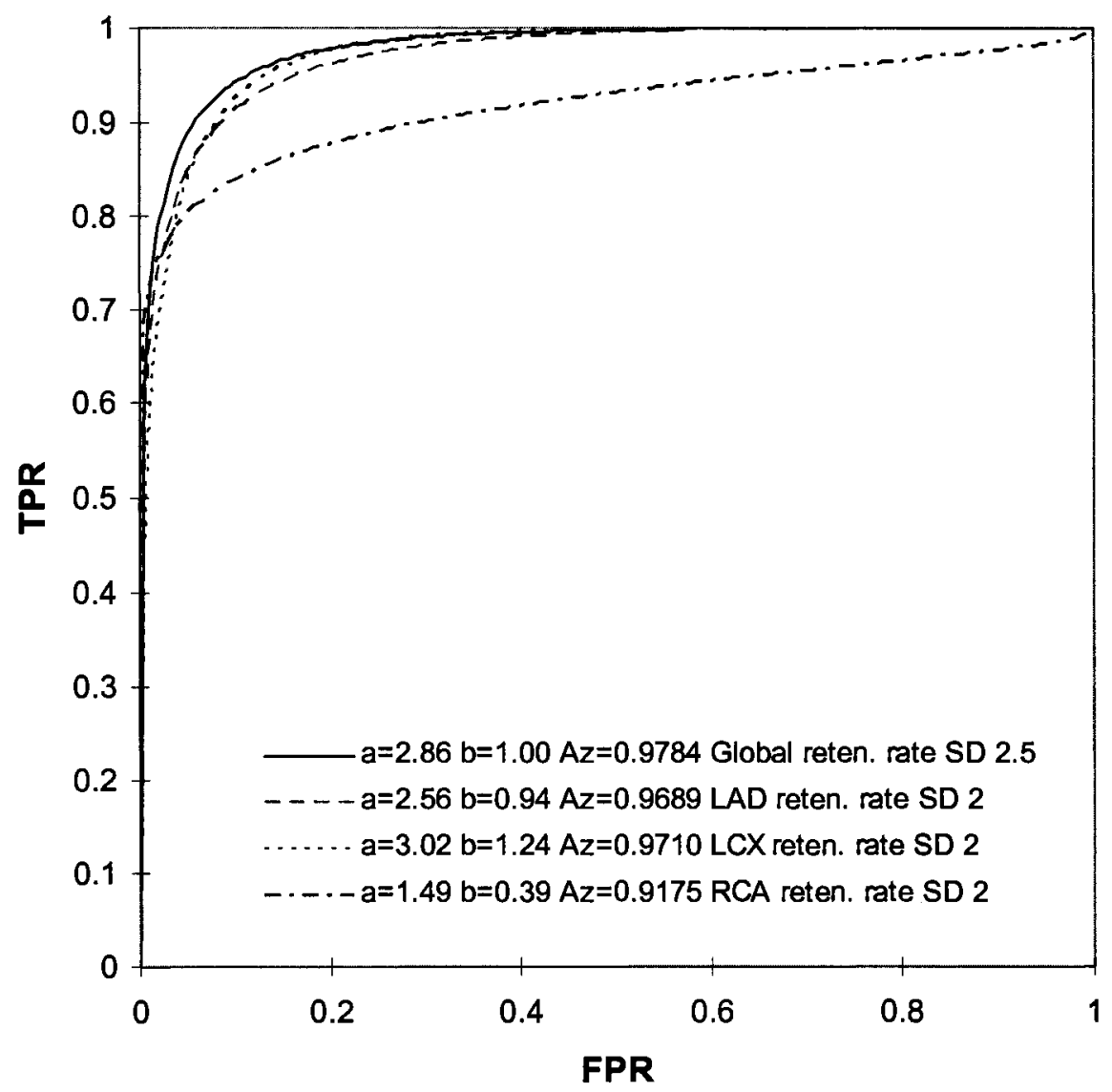

Figure 5.6: Whole LV curve at an SD threshold of 2.5, and regional ROC curves at an SD threshold of 2 , for the relative ${ }^{82} \mathrm{Rb}$ databases. 


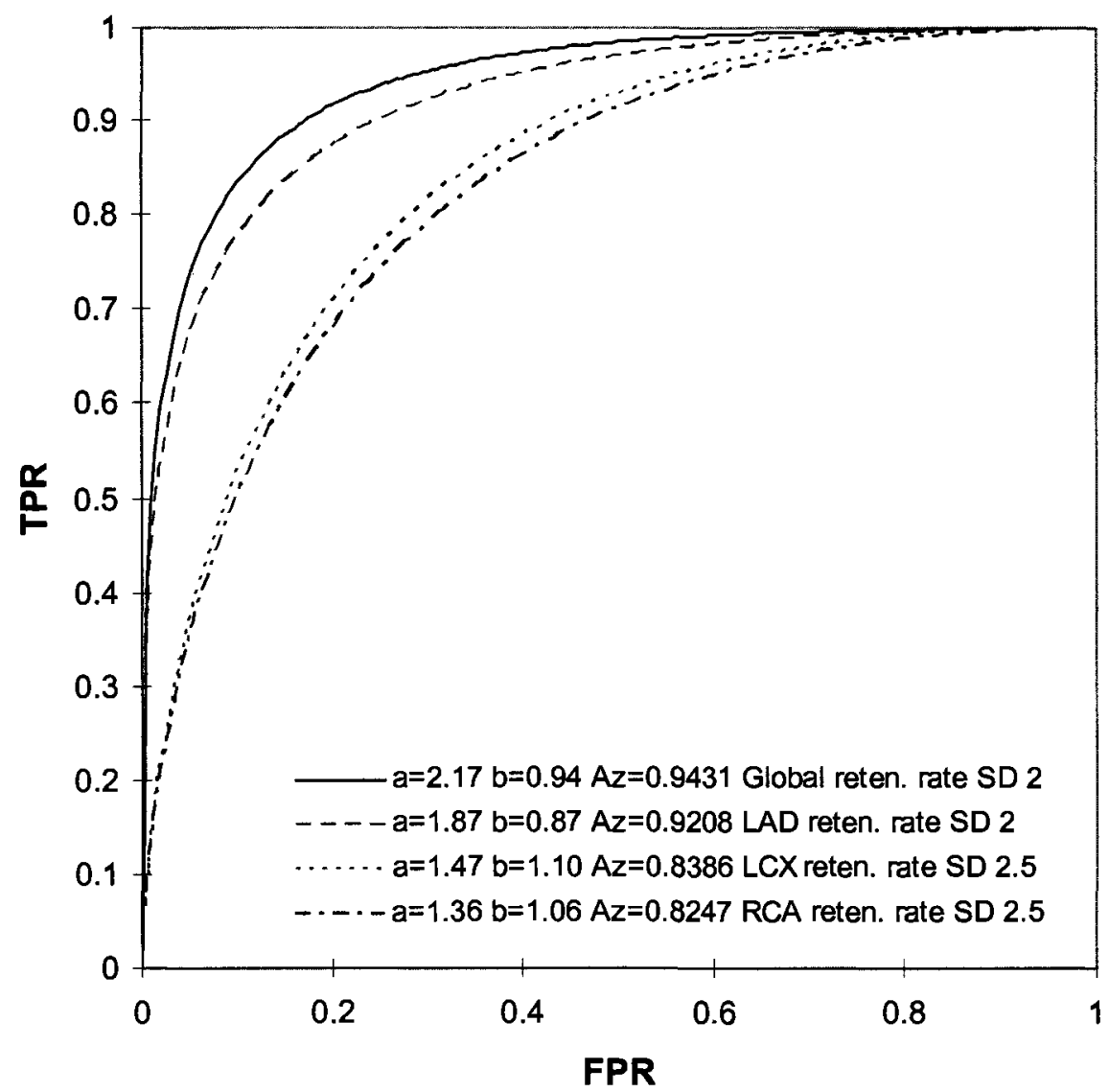

Figure 5.7: Whole LV and LAD ROC curves at an SD threshold of 2, and LCX and RCA ROC curves at an SD threshold of 2.5 for the absolute ${ }^{82}$ Rb databases.

The relative regional percent defect cutoff sizes were similar (24-37\%), and larger than the whole LV threshold (7\%). Similarly, for the absolute database the cutoffs for the LAD (9\%) and RCA (6\%) regions were larger than the whole LV (2\%), while that of the LCX was more similar (3\%). From the tables, the overall detection sensitivity and specificity of the relative and absolute retention databases can also be obtained by considering the whole $\mathrm{LV}$ region. The relative retention database has an overall sensitivity of $92 \%$ for detection of disease and a specificity of $93 \%$, whereas the absolute database has a similar SEN of $91 \%$ and a lower SPC of $81 \%$. For the relative databases, 
the overall $\operatorname{Mean}(S E N, S P C)$ for the regions is similar to the global value, with the exception of the RCA which is slightly lower, suggesting that the ability to localize disease is similar to the disease detection ability. In comparison, for the absolute databases, while the global and LAD Mean $(S E N, S P C)$ values are similar, the overall values in the LCX and RCA are lower. This is also evident from the plot of the ROC curves (Fig. 5.7), which may be an indication that a non-optimal SD threshold was selected in those territories. Also, in considering the Mean(SEN, SPC), it is observed that the relative databases have an apparent higher overall accuracy as compared to the absolute databases. This is also seemingly perceptible from the ROC curves for the relative databases, which are located closer to the top-left corner, in the more accurate region of the plot. However, the differences between the relative and absolute whole LV ROC curves were not found to be significant using a univariate z-score test comparison of the AUCs $(p=0.55)$; this is likely due to the small sample size.

\subsection{Discussion}

The stress normal limits and criteria for abnormality were evaluated for relative and quantitative ${ }^{82} \mathrm{Rb}$ normal population databases. Net retention rate, flow derived from retention, one-compartment, one-compartment DV, and one-compartment constant-DV databases were assessed. Validation was performed by comparing the results of global and regional determinations of disease in patients using the ${ }^{82} \mathrm{Rb}$ databases versus the results of the gold standard method of coronary angiography using ROC analysis. This was done to determine the most accurate ${ }^{82} \mathrm{Rb}$ PET databases to use for the detection and 
localization of $\mathrm{CAD}$. This study was limited by the robustness of the fitting algorithm used to determine the ROC curves. The fitting routine was unable to determine an appropriate solution in a number of regions and SD thresholds, limiting the selection of the optimal cutoffs, particularly for the absolute databases. This may be partially attributed to the percent defect size data, which was sparsely distributed over the range of SD values. However, it is likely due to the small sample size $(\mathrm{N}=14$ normal subjects, $N$ $=12 \mathrm{CAD}$ patients) that was used to start with, suggesting that a larger population may be necessary. These methods form a framework that can be used for more extensive evaluation of the databases in much larger patient populations in which these limitations should be overcome.

The results of this analysis indicate that the relative retention database, with a global SD threshold of 2.5 and minimum percentage defect size threshold of $7 \%$, has the greatest sensitivity (92\%) for detection of disease and the highest specificity (93\%). These values are similar to those determined in previous studies using ${ }^{82} \mathrm{Rb}$ relative perfusion imaging and expert visual analysis, as shown in Table 5.3. The average sensitivity for detecting $\mathrm{CAD}$ is $91 \%(87 \%-100 \%)$, with a corresponding average specificity of $84 \%(73 \%-$ $100 \%)$. Most of the available data was obtained with dedicated PET cameras, as in this study, with exception of the two most recent studies by Santana [37] and Sampson [63], where PET/CT was used. 
Table 5.3: Summary of literature regarding the accuracy of ${ }^{82} \mathbf{R b}$ databases for detection of CAD [19]

\begin{tabular}{lccc}
\hline \multicolumn{1}{c}{ Reference } & Scanner & Sensitivity & Specificity \\
\hline Santana et al. [37] & PET/CT & 93 & 75 \\
Sampson et al. [63] & PET/CT & 93 & 83 \\
Bateman et al. [20] & PET & 87 & 93 \\
Marwick et al. [64] & PET & 90 & 100 \\
Grover-McKay et al. [65] & PET & 100 & 73 \\
Stewart et al. [66] & PET & 83 & 86 \\
Go et al. [67] & PET & 93 & 78 \\
\hline
\end{tabular}

The work by Bateman et al. was performed using a similar PET system to the one used in this study. The sensitivity (92\%) determined in this study is higher than that found by Bateman (87\%), while the specificity $(93 \%)$ is identical at a relative global SD threshold of 2.5. Regionally, Bateman et al. found the following sensitivity and specificity values: $87 \%$ SEN and $91 \%$ SPC in the LAD, $71 \%$ SEN and $91 \%$ SPC in the LCX, and $74 \%$ SEN with $100 \%$ SPC in the RCA [20]. It is interesting to note that the values obtained in the RCA in the present study ( $73 \%$ SEN, $99 \%$ SPC) are very similar to the ones presented by Bateman; however, the overall sensitivity and specificity values obtained for the relative retention database in this study (Table 6.1) are higher than the results of Bateman. It is worth mentioning that if the data is evaluated at a global SD threshold of 2 , the resultant sensitivity $(85 \%)$ is more similar to Bateman's and the specificity $(95 \%)$ remains in the same range. Some of the differences in the results between this study and Bateman's may be accounted for by the different methodology used to obtain the sensitivity and specificity measures; Bateman's results were obtained using visual analysis as opposed to 
the automated database method used here. Therefore, this comparison indicates that the automated technique may outperform the method of visual analysis.

In contrast to the other groups, the results presented from Santana were obtained using an automated database similar to the technique used in this research. In the present study, a similar sensitivity (92\%) and higher specificity (93\%) were achieved as compared to the results of the database developed by Santana et al. In that study, a global SD threshold of 2.5 was used in combination with a defect size threshold of $3 \%$, which is similar to the 2.5 SD cutoff and 7\% defect size used in this study. The larger defect size threshold used in this study may account for some of the differences in the two specificity values. On a regional level, the three territories were subdivided by Santana et al., resulting in SD thresholds varying between 2.5 and 3 and a defect size threshold of $10 \%$ within each of the regions. Using those thresholds, they reported sensitivity and specificity values of: $76 \%$ SEN and $70 \%$ SPC in the LAD, $79 \%$ SEN and $82 \%$ SPC in the LCX, and $76 \%$ SEN with $93 \%$ SPC in the RCA [37]. Overall, these values are lower as compared to those obtained using the relative retention database in this study (Table 5.2). This is likely a product of the varying SD thresholds used within the three major territories by Santana. Consequently, these observations suggest that the technique used in the present study may be preferable for accurate disease localization.

In considering the absolute databases, the retention rate also appears to be the most accurate method, with an SD threshold of 2 and a minimum percentage defect size cutoff of $2 \%$. This operating point corresponds to overall sensitivity and specificity values of 
$91 \%$ and $81 \%$, respectively. This sensitivity is much higher than that obtained in a previous absolute database formed with ${ }^{13} \mathrm{NH}_{3}$ PET and a 2-compartment model by Muzik et al. [21] (73\%), while the specificity is similar (80\%). However, in comparison to the relative database formed in the present study at a $2.5 \mathrm{SD}$ threshold, these results suggest that the absolute database may be slightly less specific for disease detection and localization; though, it is notable that the sensitivity is improved as compared to the relative database evaluated at a $2 \mathrm{SD}$ cutoff $(85 \%)$.

The resultant incremental value gained from the quantitative databases remains to be fully determined. For example, Di Carli et al. [19] have suggested that measurements of absolute MBF and MFR can be used to correctly identify the presence and degree of stenoses in multiple coronary vessels. This would overcome the limitation of the relative perfusion databases, which only detect the most severe stenoses, while being unable to distinguish others that may be present. While no multi-vessel or balanced CAD patients were available for this work, an example of the potential quantitative benefit is shown in Figure 5.8. Polar maps are displayed for a patient from the population previously described, with known disease in the RCA as determined from coronary angiography, evaluated with the relative retention database at an SD threshold of 2.5 and a defect size cutoff of $7 \%$ (Fig. $5.8 \mathrm{~A}$ ), and the absolute retention database at SD and defect size cutoffs of 2 and $2 \%$ (Fig. $5.8 \mathrm{~B}$ ), respectively. 


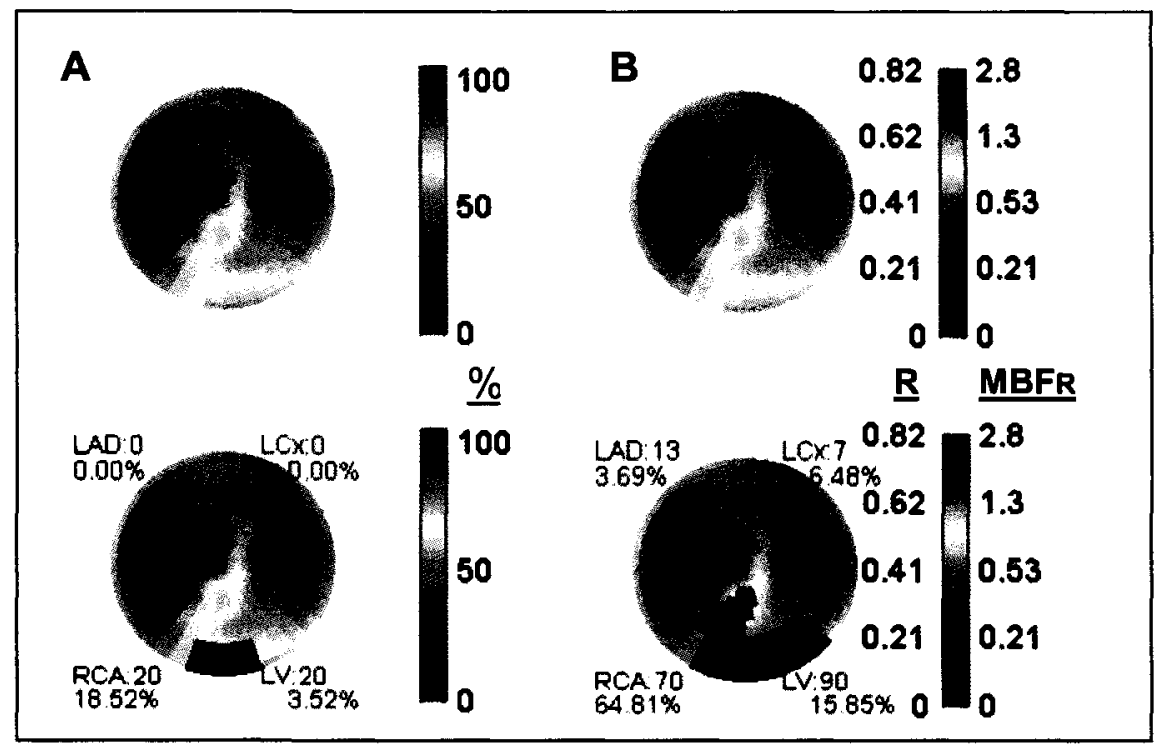

Figure 5.8: Polar maps showing the evaluation of a CAD patient with the retention database on a (A) relative scale (\%) at an SD threshold of 2.5 and (B) on an absolute scale using an SD cutoff of 2 . For the absolute evaluation, the colour-bar shows the retention rate scale on the left and the corresponding flow values on the right. The top polar maps are the same, and show that the same patient stress map that was used for evaluation in both databases. The bottom polar maps are the results of applying the databases to the patient map. The regions in dark blue are the areas that were identified as abnormal.

As can be observed from the figure, a larger region of abnormality is detected using the absolute database. Most importantly, on the absolute scale (Fig 5.8 B) the level of disease detected globally is significant at $15.85 \%$ of the $\mathrm{LV}$, since this defect size is greater than the minimum percent defect threshold of $2 \%$. In contrast, on the relative scale (Fig. 5.8 A) the region of disease would be considered to be in the normal range as the extent of $3.52 \%$ of the LV is less than the global threshold of $7 \%$. This suggests that quantitative database analysis may improve the assessment of the anatomical extent of CAD in some patients, allowing for more accurate identification of patients with severe disease, requiring aggressive intervention. Additionally, quantification may be of added utility in 
diagnosing other patient populations, such as those with early atherosclerosis and microvascular disease without evidence of coronary artery stenoses, and those with hypertrophic cardiomyopathies. In both cases, reduced MFR has been observed [16]; hence, quantitative databases evaluating this parameter would be useful for determining if the MFR reduction is significant and indicative of disease, or if the MFR value still fell within the normal range. Further evaluation of patients in these populations is required to assess the true advantage of the absolute MBF databases. 


\section{CHAPTER 6 - Conclusion}

Using compartmental modeling and a simple net retention model, ${ }^{82} \mathrm{Rb}$ has been shown to provide similar MBF and MFR values to the measurements obtained with the gold standard ${ }^{13} \mathrm{NH}_{3}$. A modified methodology for the net retention model was developed to provide a simple and robust quantification technique. As part of the development, tracerspecific extraction corrections for ${ }^{82} \mathrm{Rb}$ and ${ }^{13} \mathrm{NH}_{3}$ were derived using this model. Compared to previously published curves obtained in animal studies, the derived extraction fraction in humans was found to be higher at rest and similar at stress flows for ${ }^{82} \mathrm{Rb}$, while ${ }^{13} \mathrm{NH}_{3}$ extraction was shown to be similar at rest and lower at high flows. These results imply that ${ }^{82} \mathrm{Rb}$ and ${ }^{13} \mathrm{NH}_{3}$ extraction may be more similar than previously reported, further suggesting that ${ }^{82} \mathrm{Rb}$ may have better flow imaging properties than initially indicated.

The derived extraction corrections were used to obtain flow estimates from the net retention model for both ${ }^{82} \mathrm{Rb}$ and ${ }^{13} \mathrm{NH}_{3}$ that were comparable to the compartment model flows, but demonstrated more population variability. However, consideration of the net retention rate prior to extraction correction demonstrated considerably lower variability and improved regional uniformity, suggesting that the net retention model may be advantageous for the establishment of an absolute scale normal database for both ${ }^{82} \mathrm{Rb}$ and ${ }^{13} \mathrm{NH}_{3}$. 
The net retention model and the compartmental models were used to form population databases from a group of healthy volunteers. From these databases, lower limits of normal flow (mean - 2SD) were established for both tracers. Comparison of global and regional values of the lower limits of normal flow indicated that the net retention rate had the smallest variability for ${ }^{82} \mathrm{Rb}$ and ${ }^{13} \mathrm{NH}_{3}$, indicating that the retention model may be favourable for the detection and localization of flow reductions with both tracers. In contrast, the compartment model for ${ }^{13} \mathrm{NH}_{3}$ showed the smallest range of absolute flow values, confirming that this model yields the highest precision in quantifying absolute flow and myocardial flow reserve in normal subjects, which is consistent with previous studies. Consequently, when CAD detection is the primary objective, a robust and sensitive method is required, such as ${ }^{82} \mathrm{Rb}$ or ${ }^{13} \mathrm{NH}_{3}$ net retention. If however, accurate absolute flow quantification is desired, ${ }^{13} \mathrm{NH}_{3}$ should be used with compartmental modeling.

Through the evaluation of patients with documented CAD using the model databases, it was confirmed that the retention method is best for disease detection and localization on both relative and absolute scales. It was determined that the optimal SD threshold for establishing the lower limit of normal flow should be 2.5 globally for the relative database, and 2 for the absolute database. At this level of variability, the best combination of sensitivity and specificity for disease detection and localization was achieved.

Future work should include further validation of the derived extraction corrections for the net retention model in an independent group of normal subjects. To assess the true 
ability of the retention model to provide accurate measures in all patient populations, evaluation of the model's limitations, specifically the fixed recovery coefficient and the integration time, should be performed in a group of CAD subjects with scar and after acute myocardial infarction since these conditions may lead to different physiological behaviour in the myocardium. Development of a method to incorporate the estimation of the $\mathrm{RC}$ value by modeling, rather than setting a fixed value, may be necessary to account for these cases where the thickness of the myocardium is not constant over the entire LV. Additionally, the ${ }^{13} \mathrm{NH}_{3}$ databases should be evaluated in the same manner as the ${ }^{82} \mathrm{Rb}$ databases once appropriate normal and CAD populations are imaged with ${ }^{13} \mathrm{NH}_{3} \mathrm{PET}$. Furthermore, the databases formed from the kinetic models should be further evaluated using patient reserve polar maps as MFR may be a more accurate measure for detecting early coronary disease and multi-vessel disease. Finally, the robustness of the absolute databases should be assessed in a larger patient population using the established analysis framework to confirm their utility and added diagnostic benefit. 


\section{Appendix A - 17 Segment Model}

Segment models are used to divide the LV into smaller anatomical territories to enable regional quantification of MBF and localized assessment of disease. The segment model used in this work was the 17 segment model, where the LV was divided into 17 smaller regions within the basal (outermost on the polar map), mid and apical (innermost on the polar map) territories, as depicted in Figure B.1. The names of the regions corresponding to the numbers displayed on the polar map are listed below.

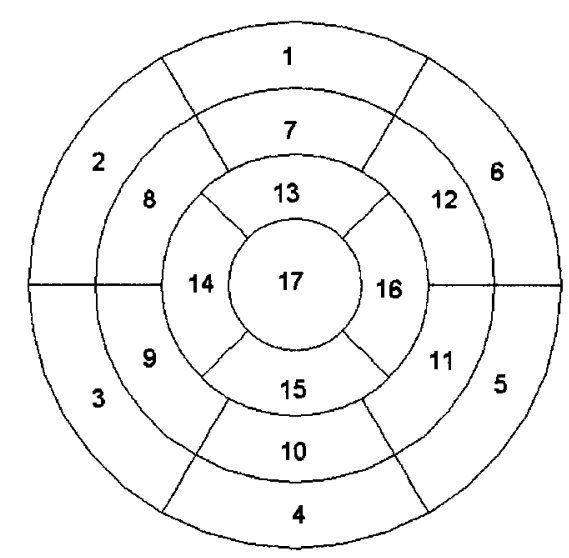

Figure A.1: Polar map of the 17 segment model.

Segments:

1. Basal Anterior

2. Basal Anteroseptal

3. Basal Inferoseptal

4. Basal Inferior

5. Basal Inferolateral

6. Basal Anterolateral

7. Mid Anterior

8. Mid Anteroseptal

9. Mid Inferoseptal
10. Mid Inferior

11. Mid Inferolateral

12. Mid Anterolateral

13. Apical Anterior

14. Apical Septal

15. Apical Inferior

16. Apical Lateral

17. Apex 


\section{References}

[1] Renaud,J.M., Lortie,M., DaSilva,J., Beanlands,R.S., deKemp,R.A., Quantification of the Normal Range of Myocardial Blood Flow and Flow Reserve with 82Rubidium Versus 13N-Ammonia PET, Conf. Rec. 2007 IEEE NSS/MIC, 4 (2007) 3141-3145.

[2] Renaud,J.M., Lortie,M., DaSilva,J., Beanlands,R.S., deKemp,R.A., Net Retention of ${ }^{82} \mathrm{Rb}$ Versus ${ }^{13} \mathrm{~N}$-Ammonia with Dynamic PET Imaging, J. Nucl. Med., 49 (2008) 75P (Abstract).

[3] Alvarez-Diez,T.M., deKemp,R., Beanlands,R., Vincent,J., Manufacture of Strontium-82/Rubidium-82 Generators and Quality Control of Rubidium-82 Chloride for Myocardial Perfusion Imaging in Patients Using Positron Emission Tomography, Appl. Radiat. Isot. 50 (1999) 1015-1023.

[4] Golanowski,L., deKemp,R.A., Beanlands,R.S., Ruddy,T.D., Precision and Bias of ${ }^{82} \mathrm{Rb}$ Kinetic Model Parameter Estimates: Computer Simulations and Dynamic PET Studies, Conf. Rec. World Congress on Medical Physics and Biomedical Engineering, (2000).

[5] deKemp,R.A., Ruddy,T.D., Hewitt,T., Dalipaj,M.M., Aung,M.T., Beanlands,R.S., Detection of Serial Changes in Absolute Myocardial Perfusion with ${ }^{82} \mathrm{Rb}$ PET, J. Nucl. Med., 41 (2001) 1426-1435.

[6] Kitsikis,A.D., deKemp,R.A., Ruddy,T.D., Beanlands,R.S., Stress ${ }^{82}$ Rb PET Normal Database for CAD Diagnosis: Development and Initial Validation, J. Nucl. Med., (2001) (Abstract). 
[7] Thompson,C.J., In Wahl,R.L., Buchanan,J.W. (Eds.), Principles and Practice of Positron Emission Tomography, Lippincott Williams \& Wilkins, Philadelphia, PA, 2002, pp. 48-64.

[8] Bacharach,S.L., In Bergmann,S.R., Sobel,B.E. (Eds.), Positron Emission Tomography of the Heart, Futura Publishing Company Inc., Mount Kisco, NY, 1992, pp. 13-44.

[9] Cherry,S.R., Dahlbom,M., PET: Physics, Instrumentation, Scanners. In Phelps,M.E. (Ed.), PET: Molecular Imaging and Its Biological Applications, Springer-Verlag, NY, 2004, pp. 1-124.

[10] Cherry,S.R., Sorenson,J.A., Phelps,M.E., Positron Emission Tomography of the Heart $3^{\text {rd }}$ Ed., Saunders - An Imprint of Elsevier Science, Philadelphia, PA, 2003.

[11] Lewellen,T., Karp,J., In Wernick,M.N., Aarsvold,J.N. (Eds.), Emission Tomography: The Fundamentals of PET and SPECT, Elsevier Academic Press, San Diego, CA, 2004, pp. 179-191.

[12] Townsend,D.W., Beyer,T., Jerin,J., Watson,C.C., Young,J., Nutt,R., The ECAT ART Scanner for Positron Emission Tomography. 1. Improvements in Performance Characteristics, Clin. Positron Imaging, 2 (1999) 5-15.

[13] deKemp,R.A, Nahmias,C., Automated Determination of the Left Ventricular Long Axis in Cardiac Positron Emission Tomography, Physiol. Meas., 17 (1996) 95-108.

[14] Mazziotta,J., Phelps,M., Plummer,D., Kuhl,D., Quantitation in Positron Emission Computed Tomography: 5. Physical-Anatomical Effects, J. Comput. Assist. Tomogr., 5 (1981) 734-743. 
[15] Hutchins,G.D., Caraher,J.M., Raylman,R.R., A Region of Interest Strategy for Minimizing Resolution Distortions in Quantitative Myocardial Perfusion Studies, J. Nucl. Med., 33 (1992) 1243-1250.

[16] deKemp,R.A., Yoshinaga,K., Beanlands,R.S.B., Will 3-Dimensional PET-CT Enable the Routine Quantification of Myocardial Blood Flow?, J. Nucl. Cardiol., 14 (2007) 380-397.

[17] Schelbert,H.R., In Marcus,M.L, Schelbert,H.R., Skorton,D.J., Wolf,G.L. (Eds.), Cardiac Imaging: A Companion to Braunwald's Heart Disease, W. B. Saunders Company, Philadelphia, PA, 1991.

[18] Klein,R., Adler,A., Beanlands,R.S., deKemp,R.A., Precision Control of Eluted Activity from a $82 \mathrm{Sr} / 82 \mathrm{Rb}$ Generator for Cardiac Positron Emission Tomography. Conf. Rec. Proceedings of the IEEE EMBS 26th Annual International Conference, 1 (2004) 1393-1396.

[19] Di Carli,M., Dorbala,S., Meserve,J., El Fakhri,G., Sitek,A., Moore,S.C., Clinical myocardial perfusion PET/CT, J. Nucl. Med., 48 (2007) 783-793.

[20] Bateman TM, Heller GV, McGhie I, Friedman,J.D., Case,J.A., Bryngelson,J.R., Hertenstein,G.K., Moutray,K.L., Reid,K., Cullom,S.J., Diagnostic accuracy of rest/stress ECG-gate Rb-82 myocardial perfusion PET: Comparison with ECGgated Tc-99m sestamibi SPECT, J. Nucl. Cardiol., 13 (2006) 24-33.

[21] Muzik,O., Duvernoy,C., Beanlands,R.S., Sawada,S., Dayanikli,F., Wolfe,E.R. Jr., Schwaiger,M., Assessment of Diagnostic Performance of Quantitative Flow Measurements in Normal Subjects and Patients with Angiographically Documented Coronary Artery Disease by Means of Nitrogen-13 Ammonia and Positron Emission Tomography, J. Am. Coll. Cardiol., 31 (1998) 534-540. 
[22] Parkash,R., deKemp,R.A., Ruddy,T.D., Kitsikis,A., Hart,R., Beauchesne,L., Williams,K., Davies,R.A., Labinaz,M., Beanlands,R.S., Potential Utility of Rubidium 82 PET Quantification in Patients with 3-Vessel Coronary Artery Disease, J. Nucl. Cardiol., 11 (2004) 440-449.

[23] Gould,K.L. Functional Measures of Coronary Artery Stenosis Severity at Cardiac Catheterization, J. Am. Coll. Cardiol., 16 (1990) 190-197.

[24] Yoshida,K., Mullani,N., Gould,K.L., Coronary Flow and Flow Reserve by PET Simplified for Clinical Applications Using Rubidium-82 or Nitrogen-13-Ammonia. J. Nucl. Med., 37 (1996) 1701-1712.

[25] Kaufmann,P., Camici,P., Myocardial Blood Flow Measurement by PET: Technical Aspects and Clinical Applications, J. Nucl. Med., 46 (2005) 75-88.

[26] Schelbert,H.R., Phelps,M.E., Huang,S.C., MacDonald,N.S., Hansen,H., Selin,C., Kuhl,D.E., N-13 Ammonia as an Indicator of Myocardial Blood Flow, Circ., 63 (1981) 1259-1272.

[27] Schelbert,H.R., Wisenberg,G., Phelps,M.E., Gould,K.L., Henze,E., Hoffman,E.J., Gomes,A., Kuhl,D.E., Noninvasive Assessment of Coronary Stenoses by Myocardial Imaging During Pharmacologic Coronary Vasodilation. VI. Detection of Coronary Artery Disease in Human Beings with Intravenous N-13 Ammonia and Positron Computed Tomography, Am. J. Cardiol., 49 (1982) 1197-1207.

[28] Hutchins,G.D., Schwaiger,M., Rosenspire,K.C., Krivokapich,J., Schelbert,H., Kuhl, D.E., Noninvasive Quantification of Regional Blood Flow in the Human Heart Using N-13 Ammonia and Dynamic Positron Emission Tomographic Imaging. J. Am. Coll. Cardiol., 15 (1990) 1032-1042. 
[29] Degrado,T.R., Hanson,M.W., Turkington,T.G., Delong,D.M., Brezinski,D.A., Vallee,J.P., Hedlund,L.W., Zhang,J., Cobb,F., Sullivan,M.J., Coleman,R.E., Estimation of Myocardial Blood Flow for Longitudinal Studies with ${ }^{13} \mathrm{~N}$-Labeled Ammonia and Positron emission tomography, J. Nucl. Cardiol., 3 (1996) 494-507.

[30] Krivokapich,J., Smith,G.T., Huang,S.C., Hoffman,E.J., Ratib,O., Phelps,M.E., Schelbert,H.R., ${ }^{13} \mathrm{~N}$ Ammonia Myocardial Imaging at Rest and with Exercise in Normal Volunteers. Quantification of Absolute Myocardial Perfusion with Dynamic Positron Emission Tomography. Circ., 80 (1989) 1328-1337.

[31] Choi,Y., Huang,S.C., Hawkins,R.A., Kuhle,W.G., Dahlbom,M., Hoh,C.K., Czernin,J., Phelps,M.E., Schlbert,H.R., A Simplified Method for Quantification of Myocardial Blood Flow Using Nitrogen-13-Ammonia and Dynamic PET. J. Nucl. Med., 34 (1993) 488-497.

[32] Nitzsche,E.U., Choi,Y., Czernin,J., Hoh,C.K., Huang,S.C., Schelbert,H.R., Noninvasive Quantification of Blood Flow in Humans. A Direct Comparison of the $\left[{ }^{13} \mathrm{~N}\right]$ Ammonia and the $\left[{ }^{15} \mathrm{O}\right]$ Water Techniques, Circ., 93 (1996) 2000-2006.

[33] Huang,S.C., Williams,B.A., Krivokapich,J., Araujo,L., Phelps,M.E., Schelbert,H.R., Rabbit Myocardial ${ }^{82} \mathrm{Rb}$ Kinetics and a Compartmental Model for Blood Flow Estimation, Am. J. Physiol., 256 (1989) H1156-1164.

[34] Herrero,P., Markham,J., Shelton,M.E., Bergmann,S.R., Implementation and Evaluation of a Two-Compartment Model for Quantification of Myocardial Perfusion with Rubidium-82 and Positron Emission Tomography, Circ. Res., 70 (1992) 496-507. 
[35] Lortie,M., Beanlands,R.S., Yoshinaga,K., Klein,R., DaSilva,J.N., deKemp,R.A., Quantification of Myocardial Blood Flow with ${ }^{82} \mathrm{Rb}$ Dynamic PET Imaging. Eur. J. Nucl. Med. Mol. Imaging., 34 (2007) 1765-1774.

[36] Lin,J.W., Sciacca,R.R., Chou,R.L., Laine,A.F., Bergmann,S.R., Quantification of Myocardial Perfusion in Human Subjects Using 82Rb and Wavelet-Based Noise Reduction, J. Nucl. Med., 42 (2001) 201-208.

[37] Santana,C.A., Folks,R.D., Garcia,E.V., Verdes,L., Sanyal,R., Hainer,J., Di Carli,M.F., Esteves,F., Quantitative ${ }^{82}$ Rb PET/CT: Development and Validation of Myocardial Perfusion Database, J. Nucl. Med., 48 (2007) 1122-1128.

[38] Koeppe,R.A., In Wahl,R.L., Buchanan,J.W. (Eds.), Principles and Practice of Positron Emission Tomography, Lippincott Williams \& Wilkins, Philadelphia, PA, 2002, pp. 65-99.

[39] Renkin,E.M., Transport of Potassium-42 from Blood to Tissue in Isolated Mammalian Skeletal Muscles, Am. J. Physiol., 197 (1959) 1205-1210.

[40] Crone,C., Permeability of Capillaries in Various Organs as Determined by Use of the Indicator Diffusion Method, Acta. Physiol. Scand., 58 (1963) 292-305.

[41] Rosenspire,K.C., Schwaiger,M., Mangner,T.J., Hutchins,G.D., Sutorik,A., Kuhl,D.E., Metabolic Fate of $\left[{ }^{13} \mathrm{~N}\right]$ Ammonia in Human and Canine Blood, J. Nucl. Med., 31 (1990) 163-167.

[42] van den Hoff,J., Burchert,W., Börner,A.R., Fricke,H., Kühnel,G., Meyer,G.J., Otto,D., Weckesser,E., Wolpers,H.G., Knapp,W.H., [1-(11)C]Acetate as a Quantitative Perfusion Tracer in Myocardial PET, J. Nucl. Med., 42 (2001) 1174 1182. 
[43] Muzik,O., Beanlands,R.S., Hutchins,G.D., Mangner,T.J., Ngyuen,N., Scwaiger,M., Validation of Nitrogen-13-Ammonia Tracer Kinetic Model for Quantification of Myocardial Blood Flow Using PET, J. Nucl. Med., 34 (1993) 83-91.

[44] Trust Region Methods for Nonlinear Minimization, Optimization Toolbox User's Guide, The Mathworks, (2000) 3.3-3.5.

[45] Gewirtz,H., Fischman,A.J., Abraham,S., Gilson,M., Strauss,H.W., Alpert,N.M., Positron Emission Tomographic Measurements of Absolute Regional Myocardial Blood Flow Permits Identification of Nonviable Myocardium in Patients with Chronic Myocardial Infarction. J. Am. Coll. Cardiol., 23 (1994) 851-859.

[46] Morris,E.D., Endres,C.J., Schmidt,K.C., Christian,B.T., Muzic,R.F., Fisher,R.E, In Wernick,M.N., Aarsvold,J.N. (Eds.), Emission Tomography: The Fundamentals of PET and SPECT, Elsevier Academic Press, San Diego, CA, 2004, pp. 400-506.

[47] Coxson,P.G., Huesman,R.H., Borland,L., Consequences of Using a Simplified Kinetic Model for Dynamic PET Data, J. Nucl. Med., 38 (1997) 660-667.

[48] Khorsand,A., Graf,S., Pirich,C., Muzik,O., Kletter,K., Dudczak,R., Maurer,G., Sochor,H., Schuster,E., Porenta,G., Assessment of Myocardial Perfusion by Dynamic N-13 Ammonia PET Imaging: Comparison of 2 Tracer Kinetic Models, J. Nucl. Cardiol., 12 (2005) 410-417.

[49] Wassenaar,R.W., deKemp,R.A., Extravascular Density Model for PET Partial Volume Correction, Conf. Rec. 2005 IEEE NSS/MIC, 3 (2005).

[50] Thompson,H.K., Starmer,F., Whalen,R.E., McIntosh,H.D., Indicator Transit Time Considered as a Gamma Variate, Circ. Res., 14 (1964) 502-515. 
[51] 1-D Data Interpolation, MATLABC 7 Function Reference, The Mathworks, (2007) 2.1770-2.1777.

[52] Bellina,C.R., Parodi,O., Camici,P., Salvadori,P.A., Taddei,L., Fusani,L., Guzzardi,R., Klassen,G.A., L'Abbate,A.L., Donato,L., Simultaneous In Vitro and In Vivo Validation of Nitrogen-13-Ammonia for the Assessment of Regional Myocardial Blood Flow, J. Nucl. Med., 31 (1990) 1335-1343.

[53] Nienaber,C.A., Ratib,O., Gambhir,S,S, Krivokapich,J., Huang,S.C., Phelps,M.E., Schelbert,H.R., A Quantitative Index of Regional Blood Flow in Canine Myocardium Derived Noninvasively with N-13-Ammonia and Dynamic Positron Emission Tomography, J. Am. Coll. Cardiol., 17 (1991) 260-269.

[54] Shah,A., Schelbert,H.R., Schwaiger,M., Henze,E., Hansen,H., Selin,C., Huang,S.C., Measurement of Regional Myocardial Blood Flow with N-13-Ammonia and Positron-Emission Tomography in Intact Dogs, J. Am. Coll. Cardiol., 5 (1985) 92100.

[55] Diamond,G.A., Forrester,J.S., Analysis of Probability as an Aid in the Clinical Diagnosis of Coronary-Artery Disease, N. Engl. J. Med., 300 (1979) 1350-1358.

[56] Wieland,B., Bida,G., Padgett,H., Hendry,G., Zippi,E., Kabalka,G., Morelle,J.L., Verbruggen,R., Ghyoot,M., In-Target Production of $\left[{ }^{13} \mathrm{~N}\right]$ Ammonia Via Proton Irradiation of Dilute Aqueous Ethanol and Acetic Acid Mixtures, Appl. Radiat. Isot., 42 (1991) 1095-1098.

[57] Epstein,N.J., Benelfassi,A., Beanlands,R.S.B., deKemp,R.A., A 82Rb Infusion System for Quantitative Perfusion Imaging with 3D PET, Appl. Radiat. Isot., 60 (2004) 921-927. 
[58] Devore,J.L., Probability and Statistics For Engineering and the Sciences $5^{\text {th }}$ Ed., Duxbury, Pacific Grove, CA, 2000.

[59] Obuchowski,N.A., Receiver Operating Characteristic Curves and Their Use in Radiology, Radiol., 229 (2003) 3-8.

[60] Metz,C.E., Herman,B.A., Shen,J-H., Maximum Likelihood Estimation of Receiver Operating Characteristic (ROC) Curves from Continuously-Distributed Data, Statist. Med., 17 (1998) 1033-1053.

[61] Metz,C.E., Pan,X., "Proper" Binormal ROC Curves: Theory and MaximumLikelihood Estimation, J. Math. Psychol., 43 (1999) 1-33.

[62] Metz,C., ROCKIT 0.9B User's Guide, The University of Chicago, 1998.

[63] Sampson,U.K., Dorbala,S., Limaye,A., Kwong,R., Di Carli,M.F., Diagnostic Accuracy of Rubidium-82 Myocardial Perfusion Imaging with Hybrid Positron Emission Tomography/Computed Tomography in the Detection of Coronary Artery Disease, J. Am. Coll. Cardiol., 49 (2007) 1052-1058.

[64] Marwick,T.H., Nemec,J.J, Stewart,W.J., Salcedo,E.E., Diagnosis of Coronary Artery Disease Using Exercise Echocardiography and Positron Emission Tomography: Comparison and Analysis of Discrepant Results, J. Am. Soc. Echocardiogr., 5 (1992) 231-238.

[65] Grover-McKay,M., Ratib,O., Schwaiger,M., Wohlgelernter,D., Araujo,L., Nienaber,C., Phelps,M., Schelbert,H.R., Detection of Coronary Artery Disease with Positron Emission Tomography and Rubidium-82, Am. Heart J., 123 (1992) 646652. 
[66] Stewart,R.E., Schwaiger,M., Molina,E., Popma,J., Gacioch,G.M., Kalus,M., Squicciarini,S., al-Aouar,Z.R., Schork,A., Kuhl,D.E., Comparison of Rubidium-82 Positron Emission Tomography and Thallium-201 SPECT Imaging for Detection of Coronary Artery Disease, Am. J. Cardiol., 67 (1991) 1303-1310.

[67] Go,R.T., Marwick,T.H., MacIntyre,W.J., Saha,G.B., Neumann,D.R., Underwood,D.A., Simpfendorfer,C.C., A Prospective Comparison of Rubidium-82 PET and Thallium-201 SPECT Myocardial Perfusion Imaging Utilizing a Single Dipyridamole Stress in the Diagnosis of Coronary Artery Disease, J. Nucl. Med., 31 (1990) 1899-1905. 